\title{
Formas traço e elementos algébricos sobre corpos Hilbertianos ${ }^{1}$
}

Fábio Alexandre de Matos

Orientadora: Profa. Dra. Ires Dias

Dissertação apresentada ao Instituto de Ciências Matemáticas e de Computação da Universidade de São Paulo, como parte dos requisitos para obtenção do título de Mestre em ciências - Area: Matemática.

\section{USP - São Carlos}

Fevereiro/2004

\footnotetext{
${ }^{1}$ Este trabalho teve suporte financeiro da Capes
} 


\section{A Comissão Julgadora:}

Profa. Dra. Ires Dias
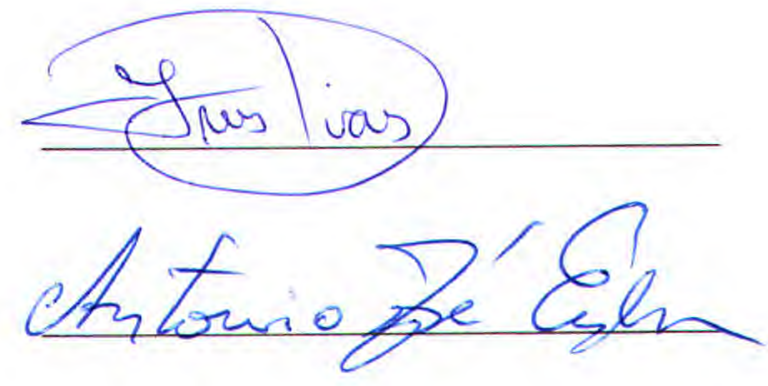

Prof. Dr. Antonio José Engler

Prof. Dr. Vitor de Oliveira Ferreira

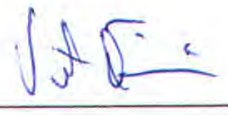


"Aos meus pais e irmãos" 


\section{Agradecimentos}

Acima de tudo a Deus, pelo dom de minha vida e por ter me dado forças suficientes a fim de superar os obstáculos e conquistar meu objetivo.

À Profa. Ires Dias, pela orientação e seriedade profissional com que conduziu este trabalho.

Aos meus pais, Maria do Carmo e Edson, pelo amor, compreensão, preocupação e apoio que sempre manifestaram e principalmente pelo esforço que fizeram para que eu tivesse oportunidade de estudo e conseguisse concluir mais esta etapa de minha vida.

Aos meus irmãos Júlio, Daniela, Fernanda e Denise, e a toda minha família pelo carinho, que de maneira indireta contribuiram sensivelmente para a realização deste trabalho.

Aos meus colegas graduação, Alex, Aline, Flávia, Grazielle, Kelly, Luci, Mariana e Mário, e aos amigos que encontrei neste instituto, que sempre me incentivaram e me ajudaram de alguma forma a ultrapassar muitos obstáculos e tornaram esta caminhada mais agradável.

A todos os professores que fizeram parte da minha vida acadêmica e sempre me incentivaram a prosseguir em meus estudos e a não desistir de meus objetivos.

Enfim, a todos aqueles que colaboraram de alguma forma para a realização deste trabalho. 


\begin{abstract}
In this work we are present a study about trace forms, scaled trace forms and algebraic elements in the Witt ring of the bilinear spaces over Hilbertian fields.
\end{abstract}




\section{Resumo}

Neste trabalho apresentamos um estudo sobre formas traço, formas traço escalar e elementos algébricos no anel de Witt dos espaços bilineares sobre corpos Hilbertinos. 


\section{Sumário}

Introduçāo

1 Espaços Bilineares, Anel de Witt e Formas Traço 2

1.1 Espaços bilineares e o Anel de Witt . . . . . . . . . . . . . . . 2

1.2 Formas Traço e Classes Algébricas . . . . . . . . . . . . . . . . . . . 9

2 Ordens 13

3 Corpos Hilbertianos

3.1 Subconjuntos de Hilbert, corpos Hilbertianos e Reduçōes . . . . . . . . . . 21

3.2 Extensões separáveis de corpos Hilbertianos . . . . . . . . . . . 26

3.3 Exemplos . . . . . . . . . . . . . . . . . . . . . . 29

4 Formas Traço Escalar sobre Corpos Hilbertianos 32

5 Formas Traço sobre Corpos de Números Algébricos 39

$\begin{array}{ll}\text { Referências Bibliográficas } & 48\end{array}$ 


\section{Introdução}

É difícil precisar quando apareceu pela primeira vez a noção de forma traço de uma extensão finita e separável de corpos. Já em 1853, Hermite e Sylvester usaram formas traço em conexão com seus estudos de raízes reais de um polinômio. Desde então a forma traço tem aparecido esporadicamente em vários contextos. Olga Taussky-Todd em [T] foi a primeira a estudar formas traço por sua própria importância e não como um ferramenta. Mais recentemente, Serre em [Se] estudou formas traço dando uma interpretação topológica para o símbolo de Hasse de formas traço. Em 1984, Conner e Perlis, em [C-P], apresentaram uma coletânia de resultados sobre formas traço de corpos de números algébricos e, neste texto, deixam algumas questões, que, a procura por suas respostas, levaram vários matemáticos a estudar formas traço e elementos algébricos em anéis de Witt.

Neste trabalho, apresentamos alguns resultados sobre formas traço sobre corpos Hilbertianos que generalizam resultados apresentados em [C-P]. Tais resultados são partes dos artigos [S2], [W] e [K] e compõem os capítulos 4 e 5 deste trabalho.

Os 3 capítulos iniciais contêm resultados preliminares necessários para a compreensão do trabalho. 
bilinear $b$ a $W \times W$, então dizemos que $\left(W, b_{\mid w}\right)$ é um subespaço não degenerado de $(V, b)$ se $\widehat{b}_{\mid W}$ é um isomorfismo.

Denotaremos por $\mathfrak{B} i \llbracket(K)$ o conjunto de todos os espaços bilineares não degenerados sobre o corpo $K$ e, no que segue, salvo menção contrária, os espaços bilineares considerados serão sempre não degenerados.

Uma isometria entre dois espaços bilineares $(V, b),\left(V^{\prime}, b^{\prime}\right)$ é um isomorfismo de espaços vetoriais $\varphi: V \rightarrow V^{\prime}$, que preserva a forma bilinear, ou seja, $b^{\prime}(\varphi(x), \varphi(y))=b(x, y)$ para todo $x, y \in V$. Quando existe uma isometria entre dois espaços bilineares $(V, b)$ e $\left(V^{\prime}, b^{\prime}\right)$ dizemos que os espaços bilineares são isométricos e denotaremos por $(V, b) \simeq\left(V^{\prime}, b^{\prime}\right)$, ou simplesmente $b \simeq b^{\prime}$ ou ainda $V \simeq V^{\prime}$.

Se $(V, b)$ é um espaço bilinear e $\left\{v_{1}, \ldots v_{n}\right\}$ é uma base de $V$ sobre $K$, então a forma bilinear é unicamente determinada pela matriz quadrada $B=\left(b_{i j}\right)=\left(b\left(v_{i}, v_{j}\right)\right)$, $1 \leq i, j \leq n$, pois para $x=\sum_{i=1}^{n} \alpha_{i} v_{i}$ e $y=\sum_{j=1}^{n} \beta_{j} v_{j}$ em $V$ temos que $b(x, y)=\sum_{i, j=1}^{n} b_{i j} \alpha_{i} \beta_{j}$. Em termos matriciais temos que se

$$
X=\left(\begin{array}{c}
\alpha_{1} \\
\vdots \\
\alpha_{n}
\end{array}\right) \text { e } Y=\left(\begin{array}{c}
\beta_{1} \\
\vdots \\
\beta_{n}
\end{array}\right)
$$

são os vetores coordenadas de $x$ e $y$ em relação à base $\left\{v_{1}, \ldots v_{n}\right\}$, então $b(x, y)=X^{t} B Y$. Reciprocamente, para cada matriz simétrica em $M_{n}(K)$, obtemos uma forma bilinear simétrica seguindo os passos descritos acima.

$O$ determinante do espaço bilinear $(V, b)$, com relação à base $\left\{v_{1}, \ldots v_{n}\right\}$, é definido como sendo o determinante da matriz $\left(b_{i j}\right)$ módulo $K^{2}$, ou seja, é o determinante de $\left(b_{i j}\right)$ a menos de quadrados, e será denotado por det $b$. É fácil verificar que o determinante de um espaço bilinear independe da escolha da base.

É imediato que um espaço bilinear $(V, b)$ é não degenerado se, e somente se $\operatorname{det} \dot{b} \neq 0$. Também é fácil ver que um espaço bilinear $(V, b)$ é não degenerado se, e somente se $\widehat{b}$ é injetora, isto é, se $b(x, y)=0$, para todo $y \in V$, então $x=0$. Resumimos estes fatos em:

Lema 1.1. Seja $(V, b)$ um espaço bilinear. Então as seguintes afirmações são equivalentes:

(i) Se $b(x, y)=0$ para todo $y \in V$, então $x=0$;

(ii) $(V, b) \in \mathfrak{B i l}(K)$;

(iii) $\operatorname{det} b \neq 0$.

Dem.: Imediata.

$\operatorname{Sejam}(V, b),\left(V^{\prime}, b^{\prime}\right) \in \mathfrak{B i l}(K)$. Definimos a soma ortogonal de $(V, b)$ e $\left(V^{\prime}, b^{\prime}\right)$ como sendo o espaço bilinear

$$
(V, b) \perp\left(V^{\prime}, b^{\prime}\right)=\left(V \oplus V^{\prime}, b \perp b^{\prime}\right),
$$


onde $b \perp b^{\prime}\left(\left(x \oplus x^{\prime}\right),\left(y \oplus y^{\prime}\right)\right)=b(x, y)+b^{\prime}\left(x^{\prime}, y^{\prime}\right)$, para todo $x, y \in V$ e $x^{\prime}, y^{\prime} \in V^{\prime}$. É fácil ver que $\left(V \oplus V^{\prime}, b \perp b^{\prime}\right) \in \mathfrak{B i l}(K)$, o qual denotaremos por $V \perp V^{\prime}$ ou ainda $b \perp b^{\prime}$.

Definimos o produto tensorial dos espaços bilineares $(V, b)$ e $\left(V^{\prime}, b^{\prime}\right)$ como sendo o espaço bilinear

$$
(V, b) \otimes\left(V^{\prime}, b^{\prime}\right)=\left(V \otimes V^{\prime}, b \otimes b^{\prime}\right)
$$

onde $b \otimes b^{\prime}\left(x \otimes x^{\prime}, y \otimes y^{\prime}\right)=b(x, y) b^{\prime}\left(x^{\prime} ; y^{\prime}\right)$, para todo $x, y \in V$ e $x^{\prime}, y^{\prime} \in V^{\prime}$. Novamente, pode-se ver que $\left(V \otimes V^{\prime}, b \otimes b^{\prime}\right) \in \mathfrak{B i l}(K)$.

Mostra-se facilmente pelas propriedades de produto tensorial e soma direta que estas operações são comutativas, associativas e que o produto tensorial se distribui com relação à soma ortogonal. Além disso, estas operações são compatíveis com relação a isometrias, ou seja, $(V, b) \simeq\left(V^{\prime}, b^{\prime}\right)$ e $\left(V_{1}, b_{1}\right) \simeq\left(V_{1}^{\prime}, b_{1}^{\prime}\right)$, então

$$
\left(V \oplus V_{1}, b \perp b_{1}\right) \simeq\left(V^{\prime} \oplus V_{1}^{\prime}, b^{\prime} \perp b_{1}^{\prime}\right) \text { e }\left(V \otimes V_{1}, b \otimes b_{1}\right) \simeq\left(V^{\prime} \otimes V_{1}^{\prime}, b^{\prime} \otimes b_{1}^{\prime}\right)
$$

Sejam $(V, b)$ um espaço bilinear e $\left(V_{1}, b_{1}\right)$ um subespaço de $(V, b)$. O conjunto $V_{1}^{\perp}=\left\{x \in V ; b(x, y)=0\right.$ para todo $\left.y \in V_{1}\right\}$ é chamado o complemento ortogonal de $V_{1}$ em $V$ com relação à forma bilinear $b$. Note que $(V, b) \in \mathfrak{B} i l(K)$ se, e somente se $V^{\perp}=\{0\}$

Seja $(V, b)$ um espaço bilinear. Um subespaço $U \subseteq V$ é dito ser totalmente isotrópico se $U \subseteq U^{\perp}$ e é dito ser um subespaço isotrópico maximal ou metabolizador se $U=U^{\perp}$. Um elemento $v \in V-\{0\}$ é dito ser isotrópico se o subespaço $U=K v$ de $V$ é totalmente isotrópico. Um elemento $v \in V$ é dito ser anisotrópico se o subespaço $U=K v$ de $V$ é não degenerado. Ainda, dizemos que $(V, b)$ é um espaço bilinear isotrópico se $V$ contém um vetor isotrópico. Se todos os elementos de $V$ são anisotrópicos, dizemos que $(V, b)$ é um espaço bilinear anisotrópico.

Lema 1.2. Se $\left(W, b_{\mid W}\right)$ é um subespaço não degenerado de um espaço bilinear $(V, b)$, então $V=W \perp W^{\perp}$.

Dem.: Da definição de complemento ortogonal, é suficiente mostrar que $V=W \oplus W^{\perp}$. Como $W$ é não degenerado, temos que $\widehat{b}_{1 w}: W \rightarrow W^{*}$ é um ismorfismo. Se $x \in W \cap W^{\perp}$, então $\widehat{b}_{\mid W}(x) \in W$ e $x \in W^{\perp}$. Logo, para todo $y \in W$, temos que $\left.\left(\widehat{b}_{\mid W} x\right)\right)(y)=b(x, y)=0$, o que mostra que $x \in \operatorname{Ker}\left(\widehat{b}_{\mid W}\right)=\{0\}$. Assim, $W \cap W^{\perp}=0$. Dado $x \in V$, temos que $b_{x \mid W} \in W^{*}$ e como $\left(W, b_{\mid W}\right)$ é não degenerado, existe $y \in W$ tal que $b_{x \mid W}=\widehat{b}_{\mid W}(y)$. Logo, para todo $z \in W$, temos

$$
b(x, z)=b_{x}(z)=b_{x \mid W}(z)=\left(\widehat{b}_{\mid W}(y)\right)(z)=b(y, z) \text {, ou seja, } x-y \in W^{\perp} .
$$

Além disso, $x=y+(x-y)$, com $y \in W$ e $(x-y) \in W^{\perp}$, de onde segue que $V=W+W^{\perp}$.

Dizemos que um espaço bilinear $(V, b)$ é próprio se existe $v \in V$ tal que $b(v, v) \neq 0$. Caso contrário, diremos que $(V, b)$ é impróprio. 
Lema 1.3. Seja $(V, b) \in \mathfrak{B i l}(K)$ próprio tal que $\operatorname{dim} V \geq 2$. Então $(V, b)$ possui um subespaço próprio não degenerado de dimensão 2 .

Dem.: Sejam $v \in V$ tal que $b(v, v) \neq 0$ e $W=K v \subseteq V$. Então $\left(W, b_{\text {|w }}\right)$ é não degenerado e do Lema 1.2 temos que $(V, b)=\left(W, b_{\mid W}\right) \perp\left(W_{1}, b_{\mid W_{1}}\right)$, onde $W_{1}=W^{\perp}$ também é não degenerado, pois $(V, b) \in \mathfrak{B i l}(K)$. Se $\left(W_{1}, b_{i W_{1}}\right)$ é próprio, então usando novamente o argumento acima, temos que existe $y \in W_{1}$ tal que $b_{w_{1}}(y, y)=b(y, y) \neq 0$. Logo, se $W_{2}$ é o subespaço gerado por $y$ temos que $(V, b)=\left(W, b_{\mid W}\right) \perp\left(W_{2}, b_{\left.\right|_{2}}\right) \perp\left(W_{3}, b_{\left.\right|_{W_{3}}}\right)$ e $\left(W, b_{\left.\right|_{W}}\right) \perp\left(W_{2}, b_{W_{2}}\right)$ é o subespaço próprio não degenerado de dimensão 2 procurado. Se $\left(W_{1}, b_{W_{1}}\right)$ é impróprio, desde $\left(W_{1}, b_{\left.\right|_{W_{1}}}\right)$ que é não degenerado, existem $z, t \in W_{1}$ tais que $b(z, t) \neq 0$. Tomando $w_{2}=v+z$ e $w_{3}=v+\lambda t$, onde $\lambda=-\frac{b(v, v)}{b(z, t)}, W_{2}=K w_{2} \mathrm{e}$ $W_{3}=K w_{3}$, temos que $\left(W_{2} \oplus W_{3}, b_{W_{2} \oplus W_{3}}\right)$ é um subespaço próprio não degenerado de $(V, b)$ de dimensão 2 , pois a matriz de $b_{\left.\right|_{2} \oplus W_{3}}$ com relação à base $\left\{w_{2}, w_{3}\right\}$ é

$$
\left(b_{i j}\right)=\left(b\left(w_{i}, w_{j}\right)\right)=\left(\begin{array}{cc}
b(v, v)+b(z, z) & 0 \\
0 & b(v, v)+\lambda^{2} b(t, t)
\end{array}\right)=\left(\begin{array}{cc}
b(v, v) & 0 \\
0 & b(v, v)
\end{array}\right)
$$

que é inversível, o que demonstra o lema.

Seja $(V, b) \in \mathfrak{B i}(K)$, dizemos que $(V, b)$ é diagonalizável, se $V$ possui uma base ortogonal com relação à forma bilinear $b$.

Observação 1.4. Seja $(V, b) \in \mathfrak{B i l}(K)$ próprio, com $\operatorname{dim} V \geq 2$. Então da demonstração do lema anterior, temos que o subespaço próprio não degenerado de dimensão 2 de $V$ tem base ortogonal.

O próximo resultado garante que todo $(V, b) \in \mathfrak{B i l}(K)$ próprio possui uma base ortogonal.

Teorema 1.5. Seja $(V, b) \in \mathfrak{B i}(K)$ próprio. Entāo $(V, b)$ é a soma ortogonal de subespaços de dimensão 1 , ou seja, $(V, b)$ é diagonalizável.

Dem.: A demonstração será feita por indução sobre a dimensão de $V$. Se $\operatorname{dim} V=1$ nada temos a demonstrar. Assumiremos que $\operatorname{dim} V \geq 2$. Desde que $(V, b)$ é próprio, do Lema 1.2 e da Observação 1.4 segue que

$$
(V, b)=\left(W_{1}, b_{1 W_{1}}\right) \perp\left(W_{2}, b_{W_{2}}\right) \perp\left(W_{3}, b_{\mid W_{3}}\right), \operatorname{com} \operatorname{dim} W_{1}=\operatorname{dim} W_{2}=1
$$

Considerando $\left(V_{1}, b_{1}\right)=\left(W_{2}, b_{W_{2}}\right) \perp\left(W_{3}, b_{W_{3}}\right)$, temos que $\left(V_{1}, b_{1}\right)$ é próprio, pois $W_{2}$ é gerado por um elemento $w_{2}$ tal que $b\left(w_{2}, w_{2}\right) \neq 0$. Por hipótese de indução, temos que $\left(V_{1}, b_{1}\right)$ admite uma base ortogonal que, juntamente com $w_{1}$, o gerador de $W_{1}$, formam uma base ortogonal para $(V, b)$.

Sejam $(V, b) \in \mathfrak{B i l}(K)$ próprio, $\left\{v_{1}, \ldots, v_{n}\right\}$ uma base ortogonal de $V$ e $\alpha_{i}=b\left(v_{i}, v_{i}\right)$, para $1 \leq i \leq n$. Neste caso, denotaremos $(V, b)$ e/ou a forma bilinear $b$ por $\left\langle\alpha_{1}, \ldots, \alpha_{n}\right\rangle$. 
Observação 1.6. Se $K$ tem característica distinta de 2, então todo espaço bilinear em $\mathfrak{B i l}(K)$ é próprio e, do teorema anterior, é diagonalizável. De fato, seja $(V, b) \in \mathfrak{B i l}(K)$. Desde que $(V, b)$ é não degenerado, existem $x, y \in V$ tais que $b(x, y) \neq 0$. Mas,

$$
b(x, y)=\frac{1}{2}(b(x+y, x+y)-b(x, x)-b(y, y))
$$

de onde segue que $b(x+y, x+y), b(x, x), b(y, y)$ não são nulos simultaneamente. Assim, $b(z, z) \neq 0$, para algum $z \in\{x+y, x, y\}$, ou seja, o espaço $(V, b)$ é próprio.

Para espaços bilineares isotrópicos temos o seguinte resultado que nos será útil.

Proposição 1.7. Sejam $K$ um corpo de caracterítistica distinta de 2 e $(V, b) \in \mathfrak{B i l}(K)$ isotrópico. Então existe um subespaço não degenerado $\left(W, b^{\prime}\right)$ de $(V, b)$ tal que $b \simeq\langle 1,-1,\rangle \perp b^{\prime}$.

Dem.: Como $(V, b)$ é isotrópico e não degenerado, temos que existem $v, w \in V$ tais que $b(v, v)=0$ e $b(v, w) \neq 0$. Multiplicando $w$ por um escalar se necessário, podemos assumir que $b(v, w)=1$. Se $b(w, w) \neq 0$, trocando $w$ por $-\frac{b(w, w)}{2} v+w$, podemos assumir também que $b(w, w)=0$. Tomando $W_{1}=K v \oplus K w$, temos que em relação à base $\{v, w\}$ de $W_{1}$, a matriz de $b_{1}=b_{W_{1}}$ é $\left(\begin{array}{ll}0 & 1 \\ 1 & 0\end{array}\right)$. Logo $\left(W_{1}, b_{1}\right)$ é um espaço não degenerado de $(V, b)$ e, do Lema 1.2 , temos que $W=W_{1}^{\perp}$ e $b^{\prime}=b_{\mid w}$ satisfaz $b \simeq b_{1} \perp b^{\prime}$. Como $b_{1}\left(v+\frac{w}{2}, v+\frac{w}{2}\right)=1$ e $\operatorname{det}\left(\begin{array}{ll}0 & 1 \\ 1 & 0\end{array}\right)=-1$, de [Lam $]$ pag. 20, segue que $b_{1} \simeq\langle 1,-1\rangle$.

Nosso próximo objetivo é demonstrar o teorema do cancelamento de Witt para espaços bilineares. Tal resultado não vale para espaços bilineares sobre corpos de característica distinta de 2, como pode ser visto no Exemplo 1.10 à seguir.

Seja $(V, b) \in \mathfrak{B i l}(K)$. Para todo elemento anisotrópico $y \in V$, definimos a aplicação $\tau_{y}: V \rightarrow V$, por

$$
\tau_{y}(x)=x-\frac{2 b(x, y)}{b(y, y)} y, \text { para todo } x \in V .
$$

É fácil ver que, de fato, $\tau_{y}$ é uma isometria de $(V, b)$, chamada de reflexão.

Lema 1.8. Sejam $K$ um corpo com $\operatorname{car}(K) \neq 2,(V, b) \in \mathfrak{B i l}(K)$ e $x, y \in V$ elementos anisotrópicos tais que $b(x, x)=b(y, y)$. Então existe um elemento anisotrópico $z$ tal que $\tau_{z}(y)=x$.

Dem.: Desde que $b(x, x)=b(y, y) \neq 0$ e $\operatorname{car}(K) \neq 2$, segue que $b(x+y, x+y)+$ $b(x-y, x-y)=2 b(x, x)+2 b(y, y)=4 b(x, x) \neq 0$ em $K$. Sem perda da generalidade, 
podemos assumir que $b(x-y, x-y) \neq 0$, pois caso contrário podemos trocar $y$ por $-y$. Temos então que $z=x-y$ é um elemento anisotrópico de $V$ e desde que

$$
\begin{aligned}
b(x-y, x-y) & =b(x, x)+b(y, y)-2 b(x, y) \\
& =2[b(x, x)-b(x, y)] \\
& =2 b(x, x-y)
\end{aligned}
$$

temos que

$$
\tau_{z}(x)=\tau_{x-y}(x)=x-\frac{2 b(x, x-y)}{b(x-y, x-y)}(x-y)=x-(x-y)=y,
$$

como queríamos.

Teorema 1.9 (Teorema do Cancelamento de Witt). Sejam $K$ um corpo com $\operatorname{car}(K) \neq 2,\left(V_{1}, b_{1}\right),\left(V_{2}, b_{2}\right)$ e $\left(V_{3}, b_{3}\right) \in \mathfrak{B i l}(K)$ tais que $b_{1} \perp b_{2} \simeq b_{1} \perp b_{3}$. Então $b_{2} \simeq b_{3}$.

Dem.: Usando indução sobre a dimensão de $V_{1}$, é suficiente mostrarmos para o caso em que $\operatorname{dim} V_{1}=1$. Mais ainda, como $b_{1} \perp b_{2} \simeq b_{1} \perp b_{3}$, comparando as dimensões, podemos supor que $b_{1}=\langle\gamma\rangle, b_{2}=\left\langle\alpha_{2}, \ldots \alpha_{n}\right\rangle$ e $b_{3}=\left\langle\beta_{2}, \ldots \beta_{n}\right\rangle$. Sejam $x \in V_{1} \perp V_{2}$ tal que $b(x, x)=\gamma$ e $y \in V_{1} \perp V_{3}$ tal que $b(y, y)=\gamma$. Do lema anterior, existe uma isometria $\tau$ entre $V_{1} \perp V_{2}$ e $V_{1} \perp V_{3}$ tal que $\tau(x)=y$ e, como $V_{1} \cong K x$ e $V_{1} \cong K y$, temos que $\tau_{V_{2}}$ é uma isometria entre $V_{2}$ e $V_{3}$, o que mostra o teorema.

Exemplo 1.10. Sejam $K$ um corpo de característica $2,(V, b)$ um espaço bilinear sobre $K$, onde $b=\langle 1,1,1,1\rangle$ e $\left\{v_{1}, v_{2}, v_{3}, v_{4}\right\}$ a base de $V$ correspondente a esta decomposição. Tomando $x=v_{1}+v_{2}, y=v_{1}+v_{3}$, e $W=K x \oplus K y \subseteq V$, temos que a matriz de $b_{1}=b_{\mid W}$ é

$$
\left(\begin{array}{ll}
0 & 1 \\
1 & 0
\end{array}\right)
$$

Logo, $\left(W, b_{1}\right)$ é um subespaço não degenerado $(V, b)$, e do Lema 1.2 temos que $(V, b) \simeq\left(W, b_{1}\right) \perp\left(W, b_{1}\right)^{\perp}$. É fácil ver que $z=x_{1}+x_{2}+x_{3}$ e $t=x_{4}$ formam uma base para $W^{\perp}$. Ainda, com relação à esta base, $b_{2}=b_{W_{\perp}}$ tem a seguinte matriz

$$
\left(\begin{array}{ll}
1 & 0 \\
0 & 1
\end{array}\right)
$$

ou seja,

$$
\langle 1,1,1,1\rangle \simeq\langle 1,1\rangle \perp\left(\begin{array}{ll}
0 & 1 \\
1 & 0
\end{array}\right)
$$


Suponha, por um momento que podemos aplicar o teorema de cancelamento de Witt. Então existe uma isometria $\varphi$ entre os espaços $\left(U, b_{3}\right)=\langle 1,1\rangle$ e $\left(W, b_{1}\right)$. Ainda, note que para $w \in W$, temos $w=\alpha x+\beta y \in W$, com $\alpha, \beta \in K$ e $b_{1}(w, w)=\alpha^{2} b_{1}(x, x)+$ $2 \alpha \beta b_{1}(x, y)+\beta^{2} b_{1}(y, y)=0$. Se $\mathfrak{C}=\left\{u_{1}, u_{2}\right\}$ é uma base para o espaço vetorial $U$, então $1=b_{3}\left(u_{1}, u_{1}\right)=b_{1}\left(\varphi\left(u_{1}\right), \varphi\left(u_{1}\right)\right)=0$, o que é um absurdo.

Portanto, o teorema de cancelamento de Witt não vale para espaços bilineares sobre corpos de característica 2.

Vamos agora construir o anel de Witt dos espaços bilineares sobre $K$.

Seja $(V, b) \in \mathfrak{B i}(K)$. Dizemos que $(V, b)$ é Witt trivial ou metabólico se $V$ admitir um subespaço totalmente isotrópico maximal $U$.

Sejam $(V, b),\left(V^{\prime}, b^{\prime}\right) \in \mathfrak{B i r}(K)$. Dizemos que $(V, b)$ e $\left(V^{\prime}, b^{\prime}\right)$ são Witt equivalentes e denotaremos por $(V, b) \sim\left(V^{\prime}, b^{\prime}\right)$ se, e somente se $\left(V \oplus V^{\prime}, b \perp\left(-b^{\prime}\right)\right)$ é Witt trivial.

A relação "Witt equivalente" é uma relação de equivalência em $\mathfrak{B i}(K)$. De fato, se $(V, b) \in \mathfrak{B i l}(K)$, então é fácil ver que a diagonal $U=\{v+v ; v \in V\} \subseteq V \oplus V$ é um metabolizador de $(V \oplus V, b \oplus(-b))$, ou seja, a relação é reflexiva.

Sejam $(V, b),\left(V^{\prime}, b^{\prime}\right) \in \mathfrak{B i l}(K)$ tais que $(V, b) \sim\left(V^{\prime}, b^{\prime}\right)$. Logo, existe um subespaço metabolizador $N=N \oplus N^{\prime}$ de $V \oplus V^{\prime}$. Claramente $W=N^{\prime} \oplus N$ é um metabolizador de $V^{\prime} \oplus V$, ou seja, $\left(V^{\prime}, b^{\prime}\right) \sim(V, b)$, e a relação é simétrica.

$\operatorname{Sejam}\left(V_{1}, b_{1}\right),\left(V_{2}, b_{2}\right),\left(V_{3}, b_{3}\right) \in \mathfrak{B i l}(K)$ tais que $\left(V_{1}, b_{1}\right) \sim\left(V_{2}, b_{2}\right)$ e $\left(V_{2}, b_{2}\right) \sim\left(V_{3}, b_{3}\right)$. Logo existem subespaços metabolizadores $N=N_{1} \oplus N_{2}$ e $W=W_{2} \oplus W_{3}$ de $V_{1} \oplus V_{2}$ e $V_{2} \oplus V_{3}$ respectivamente. Então $N_{1} \oplus W_{3}$ é um subespaço metabolizador de $V_{1} \oplus V_{3}$, pois para todo $x_{i}, v_{i} \in V_{i}, i=1,2,3$, temos

$$
\begin{aligned}
\left(b_{1} \perp\left(-b_{3}\right)\right)\left(\left(x_{1} \oplus x_{3}\right),\left(y_{1} \oplus y_{3}\right)\right)= & b_{1}\left(x_{1}, y_{1}\right)-b_{3}\left(x_{3}, y_{3}\right) \\
= & b_{1}\left(x_{1}, y_{1}\right)-b_{2}\left(x_{2}, y_{2}\right)+b_{2}\left(x_{2}, y_{2}\right)-b_{3}\left(x_{3}, y_{3}\right) \\
= & \left(b_{1} \perp\left(-b_{2}\right)\right)\left(\left(x_{1} \oplus x_{2}, y_{1} \oplus y_{2}\right)\right. \\
& +\left(b_{2} \perp\left(-b_{3}\right)\right)\left(x_{2} \oplus x_{3}, y_{2} \oplus y_{3}\right) \\
= & 0+0=0,
\end{aligned}
$$

$\log 0$ a relação é transitiva.

Por abuso de notação, continuaremos a denotar por $(V, b)$ a classe de equivalência representada pelo espaço bilinear $(V, b)$ e por $W(K)$ o conjunto de todas as classes de equivalência.

As operações $\perp$ e $\otimes$ de $\mathfrak{B i l}(K)$ induzem de maneira natural duas operações bem definidas em $W(K)$, as quais continuaremos denotando por $\perp$ e $\otimes$.

É fácil ver que com estas operações $W(K)$ é um anel comutativo com elemento identidade $\langle 1\rangle$, chamado o anel de Witt dos espaços bilineares sobre $K$.

Em $W(K)$, o elemento neutro da adição é a classe de qualquer espaço metabólico e o oposto aditivo de $(V, b)$ é $(V,-b)$. 
Observação 1.11. Note que o espaço bilinear $\langle 1,-1\rangle$ é zero em $W(K)$, pois se $\left\{v_{1}, v_{2}\right\}$ é uma base ortogonal de $(V, b)=\langle 1,-1\rangle$, então $U=K\left(v_{1}+v_{2}\right)$ é um metabolizador de $(V, b)$, ou seja, $(V, b)$ é metabólico. Mais ainda, se $\operatorname{car}(K) \neq 2$, e $(V, b)$ é metabólico, usando indução sobre a dimensão de $V$ e a Proposição 1.7 obtemos que existe um inteiro positivo $m$, tal que $b \simeq m\langle 1,-1\rangle$.

Com relação às classes de equivalência, temos:

Teorema 1.12. Seja $K$ corpo de característica distinta de 2. Então $(V, b),\left(V_{1}, b_{1}\right)$ em $\mathfrak{B i r}(K)$ são isométricos se, e somente se $\operatorname{dim} V=\operatorname{dim} V_{1}$ e são Witt equivalentes.

Dem.: $\operatorname{Se}(V, b) \simeq\left(V_{1}, b_{1}\right)$, então claramente $\operatorname{dim} V=\operatorname{dim} V_{1}$ e $(V, b) \sim\left(V_{1}, b_{1}\right)$.

Por outro lado, se $(V, b) \sim\left(V_{1}, b_{1}\right)$, então da observação acima, temos que existe um inteiro positivo $m$ tal que $b \perp\left(-b_{1}\right) \simeq m\langle 1,-1\rangle$. Somando $b_{1}$ em ambos os lados e usando que $\left(-b_{1}\right) \perp b_{1}$ é witt trivial, da observação acima, temos que $\left(-b_{1}\right) \perp b_{1} \simeq \operatorname{dim}\left(b_{1}\right)\langle 1,-1\rangle$ e, conseqüentemente, $b \perp \operatorname{dim}\left(b_{1}\right)\langle 1,-1\rangle \simeq m\langle 1,-1\rangle \perp b_{1}$. Como $\operatorname{dim} b=\operatorname{dim} b_{1}$, obtemos que $m=\operatorname{dim} b_{1}$ e, do teorema do cancelamento de Witt, segue que $b \simeq b_{1}$, como queríamos.

Encerramos esta seção mostrando que $W(K)$ é aditivamente gerado por espaços bilineares de dimensão 1 , ou seja, todo elemento de $W(K)$ pode ser representado por um espaço bilinear que admite base ortogonal.

Teorema 1.13. O anel $W(K)$ é aditivamente gerado por espaços de dimensão 1.

Dem.: $\operatorname{Seja}(V, b) \in W(K)$. Desde que $\langle 1,-1\rangle=0$ em $W(K)$, temos que $b_{1}=b \perp\langle 1,-1\rangle$ em $W(K)$. Como $b \perp\langle 1,-1\rangle$ é um espaço bilinear próprio, o resultado segue do Teorema 1.5 .

\subsection{Formas Traço e Classes Algébricas}

Apresentaremos, nesta seção o conceito de forma traço, de forma traço escalar e de classes algébricas do anel de Witt, bem como alguns resultados sobre os mesmos, os quais serão considerados nos capítulos seguintes. Alguns conceitos, notações e resultados de extensōes de corpos serão usados sem referência podem ser encontrados em [St].

Sejam $L / K$ uma extensão finita e separável de corpos, $\bar{K}$ o fêcho algébrico de $K$ e $n=[L: K]$, o grau da extensão $L / K$. Da teoria clássica de extensões de corpos, temos que existem exatamente $n$ imersões

$$
\sigma_{i}: L \rightarrow \bar{K}
$$

que fixam o corpo $K$, ou seja, $K$ é o corpo fixado por $\left\{\sigma_{1}, \ldots, \sigma_{n}\right\}$. Ainda, se $\sigma_{i}(x)=x$ para todo $i=1, \ldots, n$, então $x \in K$. Além disso, do fato de que $L / K$ é separável, temos que tais imersões são todas distintas. 
Vamos mostrar que $\left\{\sigma_{1}, \ldots, \sigma_{n}\right\}$ é de fato um conjunto linearmente independente sobre $K$. Se $n=1$, então nada temos a fazer desde que $\sigma_{1} \neq 0$. Suponhamos que exista uma relação

$$
a_{1} \sigma_{1}+\cdots a_{r} \sigma_{r}=0
$$

com $a_{i} \in K, 1 \leq i \leq r$, não todos nulos. Escolhemos uma tal relação com $r$ o menor possível. Então $r \geq 2$, pois se $r=1$, temos $a_{1}=0$, pois $\sigma_{1} \neq 0$. Como $\sigma_{1} \neq \sigma_{2}$, temos que existe $y \in L$ tal que $\sigma_{1}(y) \neq \sigma_{2}(y)$. Desde que $\sigma_{1}$ é uma imersão, segue que se $\sigma_{1}(y)=0$, então $y=0$, e assim $\sigma_{2}(y)=0$. Logo, $\sigma_{i}(y) \neq 0$, para $i=1,2$. Além disso, para todo $x \in L$, de (1.1) temos que

$$
a_{1} \sigma_{1}(y x)+\cdots a_{r} \sigma_{r}(y x)=\left(a_{1} \sigma_{1}(y) \sigma_{1}+\cdots a_{r} \sigma_{r}(y) \sigma_{r}\right)(x)=0,
$$

ou seja,

$$
a_{1} \sigma_{1}(y) \sigma_{1}+\cdots a_{r} \sigma_{r}(y) \sigma_{r}=0 .
$$

Multiplicando (1.1) por $\sigma_{1}(y)$ e subtraindo de (1.3), obtemos

$$
\left(a_{2} \sigma_{1}(y)-a_{2} \sigma_{2}(y)\right) \sigma_{2}+\cdots\left(a_{r} \sigma_{1}(y)-a_{r} \sigma_{r}(y)\right) \sigma_{r}=0,
$$

onde $\left(a_{2} \sigma_{1}(y)-a_{2} \sigma_{2}(y)\right) \neq 0$, o que contradiz a minimalidade de $r$. Portanto o conjunto $\left\{\sigma_{1}, \ldots, \sigma_{n}\right\}$ é linearmente independente.

Para cada $x \in L$, definimos o traço de $x$ por $\operatorname{tr}_{L / K}(x)=\sum_{i=1}^{n} \sigma_{i}(x)$. Temos que $\operatorname{tr}_{L / K}(x) \in K$, pois $\sigma_{j}\left(\operatorname{tr}_{L / K}(x)\right)=\sigma_{j}\left(\sum_{i=1}^{n} \sigma_{i}(x)\right)=\sum_{i=1}^{n} \sigma_{j}\left(\sigma_{i}(x)\right)=\sum_{i=1}^{n} \sigma_{i}(x)=\operatorname{tr}_{L / K}(x)$, para todo $j=1, \ldots, n$.

Assim, $\operatorname{tr}_{L / K}$ é uma aplicação $K$-linear de $L$ em $K$, chamada de traço da extensão $L / K$. Mais ainda, desde que $\left\{\sigma_{1}, \ldots, \sigma_{n}\right\}$ são linearmente independentes, temos que a aplicação $\operatorname{tr}_{L / K}$ é não nula. Além disso, usando a teoria de extensões de corpos pode-se mostrar o seguinte resultado básico sobre o comportamento dos traços de extensões.

Lema 1.14. Sejam $F / L$ e $L / K$ extensōes separáveis e finitas de corpos. Então

$$
\operatorname{tr}_{L / K} \circ \operatorname{tr}_{F / L}=\operatorname{tr}_{F / K}
$$

Considere, agora, a aplicação $\operatorname{Tr}_{L / K}\langle 1\rangle: L \times L \rightarrow K$, definida por $\operatorname{Tr}_{L / K}\langle 1\rangle(x, y)=$ $\operatorname{tr}_{L / K}(x y)$, para todo par $(x, y) \in L \times L$. Assim definida, $\operatorname{Tr}_{L / K}\langle 1\rangle$ é claramente uma forma bilinear simétrica chamada a forma traço da extensão $L / K$.

Desde que a aplicação $\operatorname{tr}_{L / K}$ é não nula, temos que existe $z \in L$ tal que $\operatorname{tr}_{L / K}(z) \neq 0$. Assim, se $0 \neq x \in L$, é tal que $\operatorname{Tr}_{L / K}\langle 1\rangle(x, y)=\operatorname{tr}_{L / K}(x y)=0$ para todo $y \in L$, então tomando $y=x^{-1} z \in L$ temos que $\operatorname{tr}_{L / K}\left(x x^{-1} z\right)=\operatorname{tr}_{L / K}(z)=0$, que é um absurdo. Logo, se $\operatorname{Tr}_{L / K}\langle 1\rangle(x, y)=0$ para todo $y \in L$, então $x=0$. Usando o Lema 1.1 segue que a forma bilinear $\operatorname{Tr}_{L / K}\langle 1\rangle$ é não degenerada. Assim, temos: 
Proposição 1.15. Seja $L / K$ uma extensão finita e separável de corpos. Então a aplicação $\operatorname{tr}_{L / K} \in \operatorname{Hom}_{K}(L, K)$ é não nula. Além disso, a aplicação $T: L \rightarrow \operatorname{Hom}_{K}(L, K)$, definida por $x \mapsto \operatorname{Tr}_{L / K}\langle 1\rangle(x$,$) , para todo x \in L$, é um isomorfismo.

Denotaremos o espaço bilinear ( $\left.L, \operatorname{Tr}_{L / K}\langle 1\rangle\right)$ simplesmente por $\operatorname{Tr}_{L / K}\langle 1\rangle$ e por $\operatorname{Tr}(K)$ a família das classes de isometria de tais formas traço, ou seja, $(V, b) \in \mathfrak{T r}(K)$ se, e somente se existe uma extensão finita e separável de corpos $L / K$ tal que $(V, b) \simeq \operatorname{Tr}_{L / K}\langle 1\rangle$.

Um elemento $\left(V_{1}, b_{1}\right)$ de $W(K)$ é dito ser algébrico se existe uma extensão finita e separável de corpos $L / K$ tal que $\left(V_{1}, b_{1}\right)=\operatorname{Tr}_{L / K}\langle 1\rangle$ em $W(K)$, ou seja, se $(V, b)$ pode ser representado por uma forma traço de alguma extensão separável de corpos $L / K$.

Sejam $L / K$ uma extensão finita e separável e $s \in \dot{L}$. Considere a aplicação

$$
\operatorname{Tr}_{L / K}\langle s\rangle: L \times L \rightarrow K,
$$

definida por $\operatorname{Tr}_{L / K}\langle s\rangle(x, y)=\operatorname{tr}_{L / K}(s x y)$, para todo par $(x, y) \in L \times L$. Note que $\operatorname{Tr}_{L / K}\langle s\rangle$ é uma forma bilinear simétrica e, usando novamente o Lema 1.1 obtemos que $\operatorname{Tr}_{L / K}\langle s\rangle$ é não degenerada.

A forma bilinear $\operatorname{Tr}_{L / K}\langle s\rangle$ é chamada a forma traço escalar da extensão $L / K$ associada ao elemento $s \in L$.

O espaço bilinear $\left(L, \operatorname{Tr}_{L / K}\langle s\rangle\right)$ será denotada simplesmente por $\operatorname{Tr}_{L / K}\langle s\rangle$ e denotaremos por $\operatorname{TrE}(K)$ a família das classes de isometria de tais forma traço, ou seja, $(V, b) \in \mathfrak{T} \mathfrak{E}(K)$ se, e somente se existe uma extensão finita e separável de corpos $L / K$ e $s \in \dot{L}$ tal que $(V, b) \simeq \operatorname{Tr}_{L / K}\langle s\rangle$.

Note que se $s \in \dot{L}^{2}$, ou seja, $s=r^{2}$ onde $r \in \dot{L}$, temos que $\operatorname{Tr}_{L / K}\langle s\rangle=\operatorname{Tr}_{L / K}\langle 1\rangle$. De fato, tomando o isomorfismo linear $\sigma: L \rightarrow L$ dado por $\sigma(x)=r x$ para todo $x \in L$, temos que $\left(L, \operatorname{Tr}_{L / K}\langle 1\rangle\right) \simeq\left(L, \operatorname{Tr}_{L / K}\langle s\rangle\right)$. Logo, $\operatorname{Tr}_{L / K}\langle 1\rangle \simeq \operatorname{Tr}_{L / K}\langle s\rangle$, o que implica que $\operatorname{Tr}_{L / K}\langle 1\rangle=\operatorname{Tr}_{L / K}\langle s\rangle$ em $W(K)$, ou seja, $\operatorname{Tr}_{L / K}\langle s\rangle$ é algébrico.

Vale observar que se $s \notin \dot{L}^{2}$ não podemos garantir que a igualdade acima não seja verdadeira. De qualquer modo, se $s \notin L^{2}$, temos que $[L(\sqrt{s}): L]=2$ e a forma traço da extenão $L(\sqrt{s})$ de $K$ é um elemento algébrico de $W(K)$.

O próximo resultado nos dá uma ligação entre $T r_{L / K}\langle\sqrt{s}\rangle, \operatorname{Tr}_{L / K}\langle 1\rangle$ e $T r_{L / K}\langle s\rangle$.

Teorema 1.16. Sejam $K$ um corpo, $L$ uma extensão finita e separável de $K$ e $s \in \dot{L}-\dot{L}^{2}$. Em $W(K)$ temos

$$
\operatorname{Tr}_{L(\sqrt{s}) / K}\langle 1\rangle=\langle 2\rangle\left(\operatorname{Tr}_{L / K}\langle 1\rangle+\operatorname{Tr}_{L / K}\langle s\rangle\right) .
$$

Dem.: Note primeiramente que $[L(\sqrt{s}): L]=[K \otimes(L \oplus L): L]=2$. Considere o seguinte isomorfismo de $K$-espaços vetoriais $\phi: L(\sqrt{s}) \rightarrow K \otimes(L \oplus L)$ definido por $\phi(x+y(\sqrt{s}))=$ $1 \otimes(x \oplus y)$, para todo $x, y \in L$. Sejam $v=v_{1}+v_{2} \sqrt{s}, w=w_{1}+w_{2} \sqrt{s} \in L(\sqrt{s})$. Então, $v w=$ $v_{1} w_{1}+s v_{2} w_{2}+\left(v_{1} w_{2}+v_{2} w_{1}\right) \sqrt{s}$ e $\operatorname{tr}_{L(\sqrt{s}) / L}(v w)=2\left(v_{1} w_{1}+s v_{2} w_{2}\right)$. Aplicando $\operatorname{tr}_{L / K}$, do Lema 1.14 obtemos, $\operatorname{tr}_{L(\sqrt{s}) / K}(v w)=\operatorname{tr}_{L / K}\left(\operatorname{tr}_{L(\sqrt{s}) / L}(v w)\right)=\operatorname{tr}_{L / K}\left(2\left(v_{1} w_{1}+s v_{2} w_{2}\right)\right)=$ $2 \operatorname{tr}_{L / K}\left(v_{1} w_{1}\right)+\operatorname{tr}_{L / K}\left(s v_{2} w_{2}\right)$. Logo, os espaços $\left(L(\sqrt{s}), \operatorname{Tr}_{L(\sqrt{s}) / K}\langle 1\rangle\right)$ e 
$\left(K \otimes(L \oplus L), 2\left(\operatorname{Tr}_{L / K}\langle 1\rangle+\operatorname{Tr}_{L / K}\langle s\rangle\right)\right)$ são isométricos e do Teorema 1.12, temos que $\operatorname{Tr}_{L(\sqrt{s}) / K}\langle 1\rangle=\langle 2\rangle\left(\operatorname{Tr}_{L / K}\langle 1\rangle+\operatorname{Tr}_{L / K}\langle s\rangle\right)$ em $W(K)$. 


\section{Capítulo 2}

\section{Ordens}

Neste capítulo apresentaremos alguns resultados sobre corpos ordenados, que serão necessários no decorrer do trabalho. Como foge ao objetivo do trabalho, alguns resultados básicos serão apenas citados e deixamos como referência principal [S1].

Seja $K$ um corpo. Um subconjunto $P$ de $K$ é dito ser uma ordem de $K$ se:

$$
P+P \subseteq P, P \cdot P \subseteq P,-1 \notin P, \diamond K \subseteq P, \quad P \cup-P=K \text { e } P \cap-P=\{0\},
$$

onde $\diamond K=\{x \in A ; x$ é uma soma de quadrados em $K\}$.

Um corpo ordenado é um par $(K, P)$ consistindo de um corpo $K$ e uma ordem $P$ de $K$. Além disso, se $x \in P-\{0\}$, então diremos que $x$ é positivo. Se $x \in K$ é tal que $x$ é positivo em todas as ordens $P$ de $K$, então $x$ é dito ser totalmente positivo. Dizemos que $(F, R) /(K, P)$ é uma extensão de corpos ordenados se $F / K$ é uma extensão de corpos e $P \subseteq R$. Neste caso, $R$ é dita ser extensão de $P$ para $F$.

Note que todo corpo ordenado $(K, P)$ tem característica zero. De fato, se $\operatorname{car}(K)=$ $p>0$, então $-1=\underbrace{1+\ldots+1}_{p-1 \text { vezes }} \in P$.

Observação 2.1. Se $(L, R) /(K, P)$ é uma extensão finita de corpos ordenados, então $L / K$ é separável, desde que $K$ tem característica zero. Mais ainda, do Teorema do Elemento Primitivo, ver [L] pag. 243, temos que $L=K(\alpha)$, para algum $\alpha \in L$ e, se $p(X)$ é o polinômio minimal de $\alpha$ sobre $K$, então $L \cong \frac{K[X]}{(p(X))}$.

O próximo resultado nos diz quando dada uma extensão $L / K$, existe uma extensão de $P$ para $L$.

Teorema 2.2. Seja $(K, P)$ um corpo ordenado. Então:

(i) Se $L=K(\sqrt{d})$, então existe uma extensão $R$ de $P$ para $L$ se, e somente se $d \in P$.

(ii) Se $L / K$ é uma extensão finita de grau ímpar, então sempre existe uma extensão $R$ de $P$ para $L$. 
Dem.: Seja $d \in P$. Considere o semi-anel $R^{\prime}$ de $L$ gerado por $P$ e por $\diamond L$, isto é, o conjunto formado pelos elementos da forma

$$
\sum_{i=1}^{n} x_{i} y_{i}^{2}, \quad x_{i} \in P, \quad y_{i} \in L, \quad n \in \mathbb{N} .
$$

Se $-1 \in R^{\prime}$, então

$$
\begin{aligned}
-1 & =\sum_{i=1}^{n} x_{i} y_{i}^{2}=\sum_{i=1}^{n} x_{i}\left(\alpha_{i}+\beta_{i} \sqrt{d}\right)^{2} \\
& =\sum_{i=1}^{n} x_{i}\left(\alpha_{i}^{2}+2 \alpha_{i} \beta_{i} \sqrt{d}+\beta_{i}^{2} d\right)=\sum_{i=1}^{n} x_{i}\left(\alpha_{i}^{2}+\beta_{i}^{2} d\right)+\left(\sum_{i=1}^{n} 2 x_{i} \alpha_{i} \beta_{i}\right) \sqrt{d},
\end{aligned}
$$

ou seja, $-1=\sum_{i=1}^{n} x_{i}\left(\alpha_{i}^{2}+\beta_{i}^{2} d\right) \in P$, o que é uma contradição. Então, $-1 \notin R^{\prime}$. É fácil ver que $R^{\prime}+R^{\prime} \subseteq R^{\prime}, R^{\prime} \cdot R^{\prime} \subseteq R^{\prime}$, e $\diamond L \subseteq R^{\prime}$. Logo, existe uma ordem $R$ de $L$ tal que $R^{\prime} \subseteq R$.(Ver [Sch] pag 108.) Por outro lado, se existe alguma extensão $R$ de $P$ para $L$, então $\sqrt{d} \in R$ ou $-\sqrt{d} \in R$. Como $P=K \cap R$, temos que $d=( \pm \sqrt{d})^{2} \in K \cap R=P$, o que mostra a afirmação (i).

Seja $L / K$ uma extensão finita de grau ímpar. Pela Observação 2.1, $L=K(\alpha)$, para algum $\alpha \in L$. Seja $p(X) \in K[X]$, polinômio minimal de $\alpha$ sobre $K$. Vamos demonstrar a afirmação (ii) por indução sobre $n=[L: K]$. Note que se $n=1, L=K$ e nada temos a demonstrar. Suponhamos, então, que a afirmação seja verdadeira para toda extensão $F / K$ de grau ímpar $m<n$. Consideremos $R^{\prime}$ o semi-anel de $L$ gerado por $P$ e por $\diamond L$ e suponhamos que $-1 \in R^{\prime}$. Então $-1=\sum_{i \in I} \alpha_{i} f_{i}(X)^{2} \bmod (p(X))$, onde $\alpha_{i} \in P, I$ é um conjunto finito de índices e $g r\left(f_{i}(X)\right)<n=g r(p(X))$. Logo, temos que $-1=p(X) q(X)+\sum_{i \in I} \alpha_{i} f_{i}(X)^{2}$, para algum $q(X) \in K[X]$. Note que os polinômios $f_{i}(X) \in K[X]$ não são constantes, pois caso contrário, como $p(\alpha)=0$, teríamos $-1=$ $p(\alpha) q(\alpha)+\sum_{i \in I} \alpha_{i} f_{i}(\alpha)^{2}=\sum_{i \in I} \alpha_{i} \beta_{i}^{2} \in P$, o que é uma contradição.

Note, ainda, que o termo de maior grau em $\sum_{i \in I} \alpha_{i} f_{i}(X)^{2}$ é de ordem par. Como $-1=\sum_{i \in I} \alpha_{i} f_{i}(X)^{2}+p(X) q(X)$ é constante, segue que $p(X) q(X)$ tem o mesmo grau que $\sum_{i \in I} \alpha_{i} f_{i}(X)^{2}$. E assim, $q(X)$ tem grau ímpar, pois $p(X)$ tem grau ímpar. Além disso, temos que $g r(q(X))<n=g r(p(X))$, pois caso contrário teríamos $g r\left(\sum_{i \in I} \alpha_{i} f_{i}(X)^{2}\right) \geq n^{2}$, o que não é possível desde que $g r\left(f_{i}(X)\right) \leq n-1$.

Seja $r_{1}(X) \cdots r_{s}(X) \in K[X]$ uma decomposição de $q(X)$ em fatorres irredutíveis. Desde que $q(X)$ tem grau ímpar, temos que existe um fator $r_{j}(X)$ com grau $m<n$ ímpar, denotaremos tal $r_{j}(X)$ simplesmente por $r(X)$. Seja $\beta$ uma raiz de $r(X)$ e considere $F=K(\beta)$. Temos que $[F: K]=m<n$ é ímpar. Por hipótese de indução, existe uma 
extensão $S$ de $P$ para $F$. Temos então que $P \subseteq S$ e $\diamond F \subseteq S$. Mas,

$$
-1=\sum_{i \in I} \alpha_{i} f_{i}(\beta)^{2}+p(\beta) q(\beta)=\sum_{i \in I} \alpha_{i} f_{i}(\beta)^{2} \in S
$$

o que é um absurdo.

Logo, concluimos que $-1 \notin R^{\prime}$ e ainda temos que $R^{\prime}+R^{\prime} \subseteq R^{\prime}, R^{\prime} \cdot R^{\prime} \subseteq R^{\prime}$, e $\diamond L \subseteq R^{\prime}$. Logo, existe uma ordem $R$ de $L$ tal que $R^{\prime} \subseteq R$.(Ver [S1] pag. 108).

Um corpo $K$ é dito ser formalmente real se -1 não é soma de quadrados em $K$. Um corpo formalmente real $K$ é dito ser um corpo real-fechado se $K$ não admite nenhuma extensão $L / K$ algébrica própria e formalmente real . Uma extensão algébrica $L / K$ é chamada o fêcho real de $K$ se $L$ é real-fechado. Analogamente, um corpo ordenado $(K, P)$ é dito ser um corpo ordenado real-fechado se $(K, P)$ não admite nenhuma extensão $(L, R) /(K, P)$ algébrica e própria. Uma extensão algébrica $(L, R) /(K, P)$ é chamada o fêcho real ordenado de $(K, P)$, se $(L, R)$ é real-fechado.

De [S1] pag. 111, temos que todo corpo ordenado $(K, P)$ admite um fêcho real ordenado $(L, R)$, e além disso, L possui uma única ordem $R$, a saber $R=\diamond L=L^{2}$.

Seja $K$ um corpo. Dizemos que $K$ é euclidiano se $K^{2}$ é uma ordem de $K$. Note que todo corpo real-fechado é euclidiano.

Vejamos agora como estes conceito estão relacionados.

Teorema 2.3. Seja $K$ um corpo. As seguintes afirmações são equivalentes:

(i) $K$ é real-fechado.

(ii) $K$ é euclidiano e todo polinômio de grau ímpar sobre $K$ tem uma raiz em $K$.

(iii) $K$ não é algebricamente fechado, mas $K(\sqrt{-1})$ é algebricamente fechado.

Dem.: Se $K$ é real fechado, então $P=K^{2}$ é a única ordem de $K$. Logo, $K$ é euclidiano. Assuma, agora, que existe um polinômio $p(X) \in K[X]$ irredutível e de grau ímpar. Seja $L=\frac{K[X]}{(p(X))}$. Então $[L: K]$ tem grau ímpar e assim, usando o Teorema 2.2 (ii), existe uma extensão de $R$ de $P$ para $L$. Logo $L$ é formalmente real e $L / K$ é uma extensão algébrica própria, o que contradiz o fato de $K$ ser real-fechado. Logo, todo polinômio de grau impar sobre $K$ tem uma raiz em $K$, o que mostra que (i) implica em (ii).

Agora, se $K$ é euclidiano e não admite extensão algébrica de grau ímpar, então $K$ não é algebricamente fechado, pois $[K(\sqrt{-1}): K]=2$. Tomando $L=K(\sqrt{-1})$, temos que todo elemento de $L$ é um quadrado, ou seja, $L=L^{2}$, pois para $\alpha+\beta \sqrt{-1} \in \dot{L}$, temos que $x+y \sqrt{-1} \in \dot{L}$, onde $x^{2}=\frac{\alpha+\sqrt{\alpha^{2}+\beta^{2}}}{2}$ e $y^{2}=\frac{-\alpha+\sqrt{\alpha^{2}+\beta^{2}}}{2}$ é tal que $(x+y \sqrt{-1})^{2}=\alpha+\beta \sqrt{-1}$, ou seja, $L$ não possui extensão quadrática. Se existe $F / L$ extensão algébrica tal que $[F: L]=2^{n} m>1$, onde $m$ é ímpar, considere $N$ o fêcho normal de $F$ sobre $L, G=G a l(N / K)$, o grupo de Galois de $N / K, H$ o 2-Sylow subgrupo de $G$ 
e $F_{H}$ o corpo fixo por $H$. Como $[G: H]=\left[F_{H}: K\right]$ temos que $\left[F_{H}: K\right]$ tem grau ímpar. Mas, $K$ não admite extensões de grau ímpar. Logo $F_{H}=K$ e $m=1$. Assim, $G$ é um 2 -grupo e portanto solúvel. Conseqüentemente existe uma cadeia de subgrupos de $G$

$$
\{e\}=H_{0} \triangleleft H_{1} \triangleleft \cdots \triangleleft H_{r}=G,
$$

com $\left[H_{i+1}: H_{i}\right]=2$. Para cada $i=0, \ldots r$, seja $L_{i}$ o subcorpo de $N$ fixo por $H_{i}$. Então $L_{r-1}=L$ desde que $\left[H_{r}: H_{r-1}\right]=2 \mathrm{e}[L: K]=2$. Agora $\left[L_{r-2}: L_{r-1}\right]=2$, mas $L_{r-1}=L$ e $L$ não possui extensão quadrática. Logo, $L_{r-2}=L$. Seguindo este raciocínio concluimos que $N=L_{r}=L_{r-1}=\cdots=L$. Portanto $L$ é algebricamente fechado, o que mostra que (ii) implica (iii).

Finalmente assuma (iii), ou seja, $K$ não é algebricamente fechado, mas $K(\sqrt{-1})$ é algebricamente fechado. Temos então que $K$ não possui extensão algébrica de grau maior que 2 e ainda $L=K(\sqrt{-1})$ é a única extensão algébrica de $K$ de grau 2. Mais ainda, a extensão $L / K$, não pode ser ordenada, pois $-1=(\sqrt{-1})^{2} \in \dot{L}^{2}$. Resta então mostrar que $K$ é formalmente real e assim $K$ será real-fechado. Note, primeiramente, que $-1 \notin K^{2}$, pois caso contrário $L=K$. Para mostrar que -1 não é soma de quadrados em $K$ é suficiente mostrar que para todo $\alpha, \beta \in K$, temos $\alpha^{2}+\beta^{2} \in K^{2}$ e, conseqüentemente toda soma finita de quadrados será um quadrado em $K$. Se $\alpha=0$ ou $\beta=0$, claramente temos que $\alpha^{2}+\beta^{2} \in K^{2}$. Assuma, então, que $\alpha, \beta \neq 0$ e considere $f(X)=\left(X^{2}-\alpha\right)^{2}+\beta^{2} \in K[X]$. Note que em $L[X], f(X)$ se decompõe como

$$
f(X)=\left(X^{2}-\alpha-\beta \sqrt{-1}\right)\left(X^{2}-\alpha+\beta \sqrt{-1}\right) .
$$

Desde que $L$ é algebricamente fechado, temos que existem $a, b \in L$ tais que $\alpha+\beta \sqrt{-1}=a^{2}$ e $\alpha-\beta \sqrt{-1}=b^{2}$. Assim,

$$
f(X)=(X-a)(X+a)(X-b)(X+b) \in L[X] .
$$

Como $[L: K]=2$ e $f(X)$ tem grau 4, segue que $f(X)$ é redutível em $K[X]$. Além disso, como $a, b, a^{2}, b^{2} \notin K$, temos que ou $(X-a)(X+b)$ ou $(X-a)(X+b)$ é irredutível em $K[X]$. Desta forma, segue que $a b \in K$. Portanto,

$$
\alpha^{2}+\beta^{2}=(\alpha+\beta \sqrt{-1})(\alpha-1 \beta \sqrt{-1})=a^{2} b^{2} \in K^{2},
$$

como queríamos.

Colorário 2.4. Seja $K$ um corpo real-fechado. Então todo polinômio sobre $K$ se decompõe como um produto de fatores lineares e quadráticos.

Dem.: Imediata.

Sejam $(K, P)$ um corpo ordenado, $(V, b) \in W(K)$ e $n=\operatorname{dim} b$. Desde que $K$ é ordenado, segue que $K$ tem característica zero, então da Observação 1.6, temos que $(V, b)$ é diagonalizável, ou seja, $b \simeq\left\langle\alpha_{1}, \ldots, \alpha_{n}\right\rangle$. Se $m$ é o número de $\alpha_{i}$ positivos com relação à 
ordem $P$ de $K$, então o número $s=m-(n-m)=2 m-n$ é chamado a assinatura do espaço $(V, b)$ em relação à ordem $P$ e será denotado por ass $((V, b))$, ou simplesmente de $\operatorname{ass}(b)$.

Nosso próximo passo é calcular a assinatura da forma traço associada a uma extensão $L / K$. Para tanto, consideremos $(L, Q) /(K, P)$ uma extensão finita e separável, $\operatorname{Tr}_{L / K}\langle 1\rangle$ a forma traço associada à extensão $L / K$ e $(F, R)$ um fêcho real ordenado de $(K, P)$. Desde que $L / K$ é finita e separável, da Observação 2.1, temos que $L=K(\alpha)$, para algum $\alpha \in L$. Seja $p(X) \in K[X]$ o polinômio minimal de $\alpha$. Assim, $L \cong \frac{K[X]}{(p(X))}$. Considere a seguinte forma bilinear $\left(\operatorname{Tr}_{L / K}\langle 1\rangle\right)_{F}=\langle 1\rangle_{F} \otimes \operatorname{Tr}_{L / K}\langle 1\rangle$ definida sobre $0 F$-espaço vetorial $F \otimes L \cong \frac{F[X]}{(p(X))}$. Se $\left\{v_{1}, \ldots, v_{n}\right\}$ é uma base de $L$ sobre $K$, então $\left\{1 \otimes v_{1}, \ldots, 1 \otimes v_{n}\right\}$ é uma base do $F$-espaço vetorial $F \otimes L$.

Note que se $v_{i} \in L$ é tal que $\operatorname{Tr}_{L / K}\langle 1\rangle\left(v_{i}, v_{i}\right)$ é positivo com relação a ordem $P$, então $\langle 1\rangle_{F} \otimes \operatorname{Tr}_{L / K}\langle 1\rangle\left(1 \otimes v_{i}, 1 \otimes v_{i}\right)=\operatorname{Tr}_{L / K}\langle 1\rangle\left(v_{i}, v_{i}\right)$ é positivo com relação a $R$, pöis $R$ estende $P$ para $F$. Assim, temos que $\operatorname{ass}_{P}\left(\operatorname{Tr}_{L / K}\langle 1\rangle\right)=\operatorname{ass}\left(\left(\operatorname{Tr}_{L / K}\langle 1\rangle\right)_{F}\right)$. Vamos fazer uma observação sobre o corpo $\frac{F[X]}{(p(X))}$. Do corolário anterior, temos que $p(X)$ se decompõe como um produto de fatores lineares e quadráticos em $F[X]$ e é separável, desde que $L / K$ é separável. Logo, em $F[X]$, temos que

$$
p(X)=p_{1}(X) \cdots p_{m}(X),
$$

onde $\operatorname{gr}\left(p_{i}(X)\right) \leq 2$ e $p_{i}(X) \neq p_{j}(X)$ para $i \neq j$. Do Teorema Chinês do Resto, temos o seguinte isomorfismo

$$
\frac{F[X]}{(p(X))} \cong \frac{F[X]}{\left(p_{1}(X)\right)} \oplus \cdots \oplus \frac{F[X]}{\left(p_{m}(X)\right)}
$$

Alguns dos polinômios $p_{i}(X)$ são lineares, digamos $p_{1}(X), \ldots, p_{r}(X)$, enquanto os restantes $p_{r+1}(X), \ldots, p_{m}(X)$ são quadráticos. Note que o número de fatores lineares é exatamente o número de raízes de $p(X)$ em $F$. Do Teorema 2.3 temos que $E=F(\sqrt{-1})$ é algebricamente fechado e $[E: F]=2$. Assim, $\frac{F[X]}{\left(p_{i}(X)\right)}=F$, para $i=1, \ldots, r$ e $\frac{F[X]}{\left(p_{i}(X)\right)}=$ $E$, para $i=r+1, \ldots, m$. Logo, temos que

$$
\frac{F[X]}{(p(X))} \cong \underbrace{F \oplus \cdots \oplus F}_{r-\text { vezes }} \oplus \underbrace{E \oplus \cdots \oplus E}_{(m-r)-\text { vezes }} .
$$

Proposição 2.5. Seja $(K, P)$ um corpo ordenado com fêcho real $F$. Sejam $p(X) \in K[X]$ um polinômio irredutível e $L=\frac{K[X]}{(p(X))}$. Então:

$$
\operatorname{ass}_{P}\left(\operatorname{Tr}_{L / K}\langle 1\rangle\right)=r
$$

onde $r$ é o número de raízes de $p(X)$ em $F$. Em particular, o número de raízes de $p(X)$ depende somente de $P$ e não da escolha de $F$. 
Dem.: Temos que $a s s_{P}\left(\operatorname{Tr}_{L / K}\langle 1\rangle\right)=\operatorname{ass}\left(\left(\operatorname{Tr}_{L / K}\langle 1\rangle\right)_{F}\right)$ e o $F$-espaço vetorial associado a $\left(\operatorname{Tr}_{L / K}\langle 1\rangle\right)_{F}$ é

$$
F \otimes_{K} L \cong \frac{F[X]}{(p(X))} \cong \underbrace{F \oplus \cdots \oplus F}_{r-\text { vezes }} \oplus \underbrace{E \oplus \cdots \oplus E}_{(m-r)-\text { vezes }},
$$

onde $E=F(\sqrt{-1})$. Além disso, essa decomposição é ortogonal com relação à $\left(\operatorname{Tr}_{L / K}\langle 1\rangle\right)_{F}$. Logo, $\left(\operatorname{Tr}_{L / K}\langle 1\rangle\right)_{F}$ de decompõe ortogonalmente como

$$
\left(\operatorname{Tr}_{L / K}\langle 1\rangle\right)_{F}=\phi_{1} \perp \cdots \perp \phi_{r} \perp \phi_{r+1} \perp \cdots \perp \phi_{m}
$$

onde $\phi_{1}, \ldots, \phi_{\tau}$ são formas bilineares de dimensão 1 e $\phi_{\tau+1}, \ldots, \phi_{m}$ são formas bilineares de dimensão 2. Mais ainda,

$$
\operatorname{ass}\left(\operatorname{Tr}_{L / K}\langle 1\rangle_{F}\right)=\sum_{i=1}^{m} \operatorname{ass}\left(\phi_{i}\right)
$$

Se $i=1, \ldots, r$, então $\phi_{i}=\operatorname{Tr}_{F / F}\langle 1\rangle$, que é a identidade e neste caso,temos que $\operatorname{ass}\left(\phi_{i}\right)=\operatorname{ass}\left(\left(\operatorname{Tr}_{F / F}\langle 1\rangle\right)_{F}\right)=1$. Se $i=r+1, \ldots, m$, então $\phi_{i}=\operatorname{Tr}_{E / F}\langle 1\rangle$ e neste caso, temos que a matriz da forma traço $\operatorname{Tr}_{E / F}\langle 1\rangle$ é

$$
\left(\begin{array}{cc}
2 & 0 \\
0 & -2
\end{array}\right)
$$

e, conseqüentemente $\operatorname{ass}\left(\left(\operatorname{Tr}_{F_{i} / F}\langle 1\rangle\right)_{F_{i}}\right)=0$. Portanto, $\operatorname{ass}_{P}\left(\operatorname{Tr}_{L / K}\langle 1\rangle\right)=\operatorname{ass}\left(\operatorname{Tr}_{L / K}\langle 1\rangle_{F}\right)$ $=\sum_{i=1}^{m} a s s\left(\phi_{i}\right)=r$, como queríamos.

Proposição 2.6. Sejam $(K, P)$ um corpo ordenado com fêcho real $F$ e $L=K(\alpha)$ uma extensão algébrica simples. Seja $\operatorname{Tr}_{L / K}\langle 1\rangle$ a forma bilinear definida acima. Assuma que ass $_{P}\left(\operatorname{Tr}_{L / K}\langle 1\rangle\right)=r>0$. Então existem, no máximo, $r$ diferentes extensões $R_{i}$ de $P$ a $L$. Além disso, as extensões são dadas pelas diferentes imersões $\sigma_{i}: L \rightarrow F$, isto é, $R_{i}=\sigma_{i}^{-1}\left(F^{2}\right)$.

Dem.: Seja $p(X) \in K[X]$ o polinômio minimal de $\alpha$ sobre $K$. Assim, $L \cong \frac{K[X]}{(p(X))}$. Do lema anterior, temos que $r$ é exatamente o número de raízes de $p(X)$ em $F$. Além disso, $r$ é o número de $K$-imersões $\sigma_{i}: L \rightarrow F$. Considere o conjunto $S=\left\{R_{i}=\right.$ $\sigma_{i}^{-1}\left(F^{2}\right)$, para $\left.i=1, \ldots, r\right\}$. Como $F$ é real-fechado, temos que $F^{2}$ é a única ordem de $F$ e, conseqüentemente $P \subseteq F^{2}$. Ainda, temos que $\sigma_{i}(K)=K$ para todo $i=1, \ldots, r$. Logo $P \subseteq R_{i}$ para todo $i=1, \ldots, r$. Assim, $R_{i}$ são extensões de $P$ para $L$.

Suponhamos que exista outra extensão $Q$ de $P$ para $L$. Da definição das extensões $R_{i}$, temos que existem $a_{i} \in Q$ tal que $\sigma_{i}\left(a_{i}\right) \notin F^{2}$, para cada $i=1, \ldots, r$. Desta maneira, temos que $a_{i} \notin L^{2}$, desde que $L^{2} \subseteq R_{i}$, para todo $i=1, \ldots, r$. Logo, usando o Teorema 
2.2, temos que $Q$ se estende a uma ordem $Q_{1}$ de $L_{1}=L\left(\sqrt{a_{1}}\right)$. Ainda, como $Q \subseteq Q_{1}$, segue que $a_{2} \in Q \subseteq Q_{1}$ e assim usando novamente o Teorema 2.2 $Q_{1}$ se estende a uma ordem $Q_{2}$ de $L_{2}=L_{1}\left(\sqrt{a_{2}}\right)$. Seguindo esse processo, obtemos uma ordem $R$, extensão de $Q$, para $E=L\left(\sqrt{a_{1}}, \ldots, \sqrt{a_{r}}\right)$. Desde que $\operatorname{car}(K)=0, E / K$ é separável, temos da Observação 2.1 que existe um elemento primitivo $\beta \in E$ tal que $E=K(\beta)$. Seja $q(X) \in K[X]$ o polinômio minimal de $\beta$ sobre $K$. Como $\operatorname{ass}\left(\operatorname{Tr}_{L / K}\langle 1\rangle\right)>0$, da proposição anterior temos que $q(X)$ possui pelo menos um zero em $F$. Logo, existe uma $K$-imersão

$$
\sigma: E \rightarrow F
$$

Então, $\sigma_{l_{L}}=\sigma_{j}$, para algum $j$. Temos, então que

$$
\sigma_{j}\left(a_{j}\right)=\sigma\left(a_{j}\right)=\sigma\left({\sqrt{a_{j}}}^{2}\right)=\sigma\left(\sqrt{a_{j}}\right)^{2} \in F^{2},
$$

o que é uma contradição. Portanto, não existe tal extensão $Q$ de $P$, ou seja, o conjunto $S$ é formado por todas as extensōes de $P$ para $L$.

Teorema 2.7. Seja $(K, P)$ um corpo ordenado. Então

(i) Se $(L, R) /(K, P)$ é uma extensão algébrica de corpos ordenados e $F$ o fêcho real de $(K, P)$, então existe uma imersão $\sigma: L \rightarrow F$ que preserva ordem.

(ii) Se $E, F$ são fêchos reais de $(K, P)$, então existe exatamente um $K$-isomorfismo entre $E$ e $F$ que, necessariamente preserva ordem.

(iii) $\mathrm{Na}$ situação da proposição anterior, temos que as extensões $R_{i}$, para $i=1, \ldots, r$ de $P$ para $L$ são distintas.

Dem.: Considere o conjunto $S$ de todos os corpos $K^{\prime}$ tais que $K \subseteq K^{\prime} \subseteq L$ e existe uma $K$-imersão

$$
\sigma:\left(K^{\prime}, K^{\prime} \cap R\right) \rightarrow\left(F, F^{2}\right)
$$

que preserva ordem. Note que $S \neq 0$, pois $K \in S$. Logo, do Lema de Zorn, existe um elemento maximal $K_{0}^{\prime}$ de $S$. Do lema anterior, temos que $L \in S$ e, como $K_{0}^{\prime} \subseteq L$ segue que $K_{0}^{\prime}=L$. Portanto existe uma $K$-imersão $\sigma: L \rightarrow F$, o que prova (i).

Por (i) temos que existe um $K$-homomorfismo $\sigma: E \rightarrow F$. Seja $\tau: E \rightarrow F$ um outro homorfismo. Desde que $\tau\left(E^{2}\right) \subseteq F^{2}$, e $E$ e $F$ são fechos reais de $K$, temos que $\tau$ preserva ordem como $\sigma$. Seja $\alpha \in E$ com polinômio minimal $p(X)$ sobre $K$ e sejam $\alpha_{1}, \ldots, \alpha_{r}$ seus conjugados em $E$. Podemos supor que $\alpha_{1}<\alpha_{2} \cdots<\alpha_{r}$. Desde que $\sigma$ e $\tau$ preservam a única ordem de $E$, temos que

$$
\left.\sigma\left(\alpha_{1}\right)<\cdots<\sigma\left(\alpha_{r}\right) \text { e } \tau\left(\alpha_{1}\right)<\cdots<\tau_{(} \alpha_{r}\right) .
$$

Portanto, temos que $\sigma\left(\alpha_{i}\right)=\tau\left(\alpha_{i}\right)$ e em particular, $\sigma(\alpha)=\tau(\alpha)$, de onde segue (ii).

Assuma, agora, que $R_{i}=R_{j}=R$ para algum $i \neq j$. Seja $E$ o fêcho real de $(L, R)$. Por (i) as imersões $\sigma_{i} \neq \sigma_{j}$ se estendem a diferentes $K$-homorfismos $\tau_{i} \neq \tau_{j}: E \rightarrow F$. Mas isto é impossível pelo ítem (ii). Logo vale (iii). 
Teorema 2.8. Seja $(K, P)$ um corpo ordenado e $L / K$ uma extensão finita. Então para todo espaço bilinear $\langle\alpha\rangle$ sobre $L$, temos:

$$
\operatorname{ass} s_{P}\left(\operatorname{Tr}_{L / K}\langle\alpha\rangle\right)=\sum_{R \supseteq P} a s s_{R}(\langle\alpha\rangle)
$$

onde a soma é tomada sobre todas as extensões $R$ de $P$ para $L$.

Dem.: Como $L / K$ é finita e $\operatorname{car}(K)=0$ temos que $L=K(\alpha)$, para algum $\alpha \in L$. Seja $p(X) \in K[X]$ o polinômio minimal de $\alpha$ sobre $K$. Assim, $L \cong \frac{K[X]}{(p(X))}$. Temos que $\operatorname{ass}_{P}\left(\operatorname{Tr}_{L / K}\langle 1\rangle\right)=\operatorname{ass}\left(\left(\operatorname{Tr}_{L / K}\langle 1\rangle\right)_{F}\right)$ e o $F$-espaço vetorial associado a $\left(\operatorname{Tr}_{L / K}\langle 1\rangle\right)_{F}$ é $F \otimes_{K} L \cong \frac{F[X]}{(p(X))}$. Além disso,

$$
\frac{F[X]}{(p(X))} \cong F \oplus \cdots \oplus F \oplus E \oplus \cdots \oplus E
$$

onde $E=F(\sqrt{-1})$.

Usando as Proposições 2.5 e 2.6 temos que o número de extensões $R$ de $P$ para $L$ é exatamente o número de fatores $F$ na decomposição acima. Assim, se $\alpha$ é negativo na extensão $R$ de $P$ temos que $\operatorname{ass}_{R}(\langle\alpha\rangle)=-1$ e se $\alpha$ é positivo na extensão $R$ de $P$ temos que $\operatorname{ass}_{R}(\langle\alpha\rangle)=1$. Logo ass $(\langle\alpha\rangle)=0$ sobre $E$. Denote por $n_{+}$o número de fatores $F$ nos quais $\alpha$ é positivo e por $n_{-}$o número de fatores $F$ nos quais $\alpha$ é negativo. Então,

$$
\operatorname{ass}_{P}\left(\operatorname{Tr}_{L / K}\langle\alpha\rangle\right)=\operatorname{ass_{P}}\left(\operatorname{Tr}_{L / K}\langle\alpha\rangle\right)_{F}=n_{+}-n_{-}=\sum_{R \supseteq P} a s s_{R}(\langle\alpha\rangle),
$$

como queríamos. 


\section{Capítulo 3}

\section{Corpos Hilbertianos}

Os principais resultados que compõem este trabalho são sobre formas traço e formas traço escalares sobre corpos hilbertianos. Assim, neste capítulo, apresentaremos a definição e alguns resultados sobre tais corpos.

\subsection{Subconjuntos de Hilbert, corpos Hilbertianos e Reduções}

Sejam $K$ um corpo, $\mathrm{T}=\left\{T_{1}, \ldots, T_{r}\right\}$ e $\mathrm{X}=\left\{X_{1}, \ldots, X_{n}\right\}$ dois conjuntos de variáveis livres. Sejam $f_{1}(\mathrm{~T}, \mathrm{X}), \ldots, f_{m}(\mathrm{~T}, \mathrm{X})$ polinômios nas variáveis $X_{1}, \ldots, X_{n}$ com coeficientes em $K(\mathrm{~T})$, o corpo de frações do anel de polinômios $K[\mathrm{~T}]$. Assuma ainda que $f_{1}(\mathrm{~T}, \mathrm{X}), \ldots, f_{m}(\mathrm{~T}, \mathrm{X})$ são polinômios irredutíveis sobre o anel $K(\mathrm{~T})[\mathrm{X}]$. Para $0 \neq g(\mathrm{~T})$ em $K[\mathbf{T}]$, denote por $H_{K}\left(f_{1}, \ldots, f_{m} ; g\right) \circ$ conjunto das $r$-uplas $\mathbf{a}=\left(a_{1}, \ldots, a_{r}\right) \in K^{r}$ tais que $g(\mathbf{a}) \neq 0$ e $f_{1}(\mathbf{a}, \mathbf{X}), \ldots, f_{m}(\mathbf{a}, \mathbf{X})$ são irredutíveis em $K[\mathbf{X}]$. Chamamos tais conjuntos $H_{K}\left(f_{1}, \ldots, f_{m} ; g\right)$ de subconjuntos de Hilbert de $K^{r}$. Se $g=1 \in K$, então denotaremos $H_{K}\left(f_{1}, \ldots, f_{m} ; g\right)$ simplesmente por $H_{K}\left(f_{1}, \ldots, f_{m}\right)$.

Um corpo $K$ é dito ser Hilbertiano se todos os seus subconjuntos de Hilbert são não vazios.

No que segue, $\mathrm{T}, \mathrm{X}$ representam conjuntos de variáveis, enquanto $T, X, Y, T_{i}, X_{i}$ são variáveis únicas. A primeira observação que temos é

Proposição 3.1. Todo corpo Hilbertiano é infinito.

Dem.: Sejam $K$ um corpo Hilbertiano e $H_{K}(f ; g)$, onde $f \in K(T)[X]$ é irredutível e $0 \neq g \in K[X]$, um subconjunto de Hilbert de K. Suponhamos que o subconjunto $H_{K}(f ; g) \subseteq K$ é finito, ou seja, $H_{K}(f ; g)=\left\{\alpha_{1}, \ldots, \alpha_{n}\right\}$. Considere o polinômio $h(X)=$ $\left(X-\alpha_{1}\right) \cdots\left(X-\alpha_{n}\right) \in K[X]$ e o subconjunto de hilbert $H_{K}(f ; h g)$. Note que $h g \neq 0$ em $K[X]$. Seja $\alpha \in K$. Se $\alpha \in H_{K}(f ; g)$, então $h(\alpha)=0$ e assim $h g(\alpha)=0$. Logo $\alpha \notin H_{K}(f ; h g)$. Se $\alpha \notin H_{K}(f ; g)$, então ou $f(\alpha, X)$ é redutível em $K[X]$ ou $g(\alpha)=0$. 
Mas em ambos os casos segue que $\alpha \notin H_{K}(f ; h g)$. Logo, $H_{K}(f ; h g)=\emptyset$, o que contradiz o fato de $K$ ser hilbertiano. Portanto $H_{K}(f ; g) \subseteq K$ é infinito, o que implica que o corpo $K$ é infinito.

Antes de prosseguirmos, vamos apresentar uma aplicação que é ferramenta útil na demonstração de alguns resultados no decorrer do capítulo.

Seja $S_{K}(n, d)$ o conjunto de todos os polinômios $f \in K\left[X_{1}, \ldots, X_{n}\right]$ com $g r_{X_{j}}(f)<d$, $j=1, \ldots, n$. Considere a aplicação:

$$
S_{d}: S_{K}(n, d) \rightarrow S_{K}\left(1, d^{n}\right)
$$

definida $S_{d}\left(X_{j}\right)=Y^{d^{j-1}}, j=1, \ldots, n$. A aplicação $S_{d}$ é chamada de substituição de Kronecker.

Note que os coeficientes de $f$ coincidem com os coeficientes de $S_{d}(f)$, ou seja, se $f\left(X_{1}, \ldots, X_{n}\right)=\sum_{i} a_{i} X_{1}^{i(1)} \cdots X_{n}^{i(n)}$, onde $a_{i} \in K$, então,

$$
S_{d}(f)=\sum_{i} a_{i}\left(Y^{d^{1-1}}\right)^{i(1)} \cdots\left(Y^{d^{n-1}}\right)^{i(n)}=\sum_{i} a_{i} Y^{i(1)+i(2) d+\cdots+i(n) d^{n-1}} .
$$

Ainda, se tivermos $f, g \in S_{K}(n, d)$ tais que $f g \in S_{K}(n, d)$, então $S_{d}(f g)=S_{d}(f) S_{d}(g)$.

Sejam $A=\{0,1, \ldots, d-1\}, B=\left\{0,1, \ldots, d^{n}-1\right\} \subseteq \mathbb{Z}$ e considere a aplicação $\varphi: A^{n} \rightarrow B$, dada por $\varphi\left(a_{1}, \ldots, a_{n}\right)=a_{1}+a_{2} d+\cdots+a_{n} d^{n-1}$. Note que $\varphi$ é claramente sobrejetora e usando que um número inteiro se escreve de maneira única em uma dada base, temos que $\varphi$ é injetora. Note que para cada $i$, temos $i(1)+i(2) d+\cdots+i(n) d^{n-1}=$ $\varphi(i(1), \ldots, i(n))$. Agora, usando igualdade de polinômios e o fato que $\varphi$ é bijetora, obtemos que $S_{d}$ é bijetora, ou seja, existe uma correspondência biunívoca entre $S_{K}(n, d)$ e $S_{K}\left(1, d^{n}\right)$.

Não é uma tarefa fácil analisar se um dado corpo é Hilbertiano. Apresentaremos, então, alguns resultados de reduções que nos permitem analisar mais facilmente se um dado corpo é Hilbertiano.

Lema 3.2. Cada conjunto de Hilbert $H_{K}\left(f_{1}, \ldots, f_{m} ; g\right)$ de $K$ contém um conjunto de Hilbert da forma $H_{K}\left(f_{1}^{\prime}, \ldots, f_{m}^{\prime} ; g^{\prime}\right)$ onde cada $f_{i}^{\prime}$ é irredutível no anel $K[\mathrm{~T}, \mathrm{X}]$, para $i=1, \ldots m$.

Dem.: Para cada $i=1, \ldots, m$, temos que $f_{i}(\mathrm{~T}, \mathrm{X}) \in K(\mathrm{~T})[\mathrm{X}]$, ou seja, $f_{i}(\mathrm{~T}, \mathrm{X})=$ $\sum_{j} \frac{\alpha_{i j}(\mathrm{~T})}{\beta_{i j}(\mathrm{~T})} X_{1}^{j(1)} \cdots X_{n}^{j(n)}$, onde $\alpha_{i j}(\mathrm{~T}), \beta_{i j}(\mathrm{~T}) \in K[\mathrm{~T}]$. Seja $g_{i}(\mathrm{~T})=\mathrm{mmc}_{1 \leq j \leq j(i)}\left\{\beta_{i j}(\mathrm{~T})\right\}$ Então $g_{i}(\mathrm{~T}) f_{i}(\mathrm{~T}, \mathbf{X}) \in K[\mathbf{T}, \mathbf{X}]$. Considere agora, $d_{i}(\mathbf{T})$ o máximo divisor comum dos coeficientes de $g_{i}(\mathrm{~T}) f_{i}(\mathrm{~T}, \mathbf{X})$ no anel $K(\mathbf{T})[\mathbf{X}]$ e tome $f_{i}^{\prime}=\frac{g_{i}(\mathbf{T}) f_{i}(\mathrm{~T}, \mathbf{X})}{d_{i}(\mathrm{~T})} \in K[\mathbf{T}, \mathbf{X}] . \dot{E}$ imediato que $f_{i}^{\prime}$ é um polinômio irredutível em $K[\mathrm{~T}, \mathrm{X}]$.

Sejam $g^{\prime}=g \cdot g_{1} \cdot d_{1} \cdots g_{m} \cdot d_{m}$ e $\mathbf{a}=\left(a_{1}, \ldots, a_{r}\right) \in H_{K}\left(f_{1}^{\prime}, \ldots, f_{m}^{\prime} ; g^{\prime}\right)$. Então, $f_{i}^{\prime}(\mathbf{a}, \mathbf{X})$ é irredutivel em $K[\mathbf{X}]$, para cada $i=1, \ldots m$ e $g^{\prime}(\mathbf{a}) \neq 0$. Note que $f_{i}(\mathrm{a}, \mathrm{X})$ é um 
polinômio irredutível em $K[X]$, pois $f_{i}^{\prime}(\mathbf{a}, \mathrm{X})=\frac{g_{i}(\mathbf{a}) f_{i}(\mathrm{~T} X)}{d_{i}(\mathrm{a})}=\alpha_{i} f_{i}(\mathrm{a}, \mathrm{X})$, onde $\alpha_{i} \in K$. Além disso, como $g^{\prime}(\mathbf{a})=g(\mathbf{a}) g_{1}(\mathbf{a}) d_{1}(\mathbf{a}) \cdots g_{m}(\mathbf{a}) d_{m}(\mathbf{a}) \neq 0$, temos $g(\mathbf{a}) \neq 0$. Portanto, $a \in H_{K}\left(f_{1}, \ldots, f_{m} ; g\right)$ : ou seja, $H_{K}\left(f_{1}^{\prime}, \ldots, f_{m}^{\prime} ; g^{\prime}\right) \subseteq H_{K}\left(f_{1}, \ldots, f_{m} ; g\right)$.

Com isso, temos o primeiro resultado de redução.

Proposição 3.3. Se cada subconjunto de Hilbert de $K$ da forma $H_{K}\left(f_{1}, \ldots, f_{m} ; g\right)$ onde cada $f_{i}$ é irredutível em $K\left[T, X_{1}, \ldots, X_{n}\right]$, para $i=1, \ldots, m$ é não vazio, então $K$ é Hilbertiano.

Dem.: Sejam $f_{1}, \ldots, f_{m} \in K(\mathrm{~T})[\mathbf{X}]$ polinômios irredutíveis e $0 \neq g$ em $K[\mathrm{~T}]$. Usando o Lema 3.2 temos que existem polinômios irredutíveis $f_{1}^{\prime}, \ldots, f_{m}^{\prime} \in K[\mathrm{~T}, \mathrm{X}]$ e $0 \neq g^{\prime}$ em $K[\mathrm{~T}]$ tais que $H_{K}\left(f_{1}^{\prime}, \ldots, f_{m}^{\prime} ; g^{\prime}\right) \subseteq H_{K}\left(f_{1}, \ldots, f_{m} ; g\right)$. Vamos agora dividir as variáveis $\mathrm{T}$ e X em dois conjuntos: um contendo $T_{1}$ e o outro contendo $T_{2}, \ldots, T_{r}, X_{1}, \ldots, X_{n}$. Por hipótese, existe $a_{1} \in K$ tal que para cada $i=1 \ldots, m, f_{i}^{\prime}\left(a_{1}, T_{2}, \ldots, T_{r}, \mathbf{X}\right)$ é irredutível em $K\left[T_{2}, \ldots, T_{r}, X_{1}, \ldots, X_{n}\right]$ e $g^{\prime}\left(a_{1}, T_{2}, \ldots, T_{r}\right) \neq 0$. Repetindo esse processo $r$ vezes, temos que existem elementos $a_{1}, \ldots, a_{r} \in K$ tais que para cada $i=1, \ldots, m, f_{i}^{\prime}\left(a_{1}, a_{2}, \ldots, a_{\tau}, \mathrm{X}\right)$ é irredutível em $K[\mathrm{X}]$ e $g^{\prime}\left(a_{1}, a_{2}, \ldots, a_{r}\right) \neq 0$. Logo, $H_{K}\left(f_{1}^{\prime}, \ldots, f_{m}^{\prime} ; g^{\prime}\right) \neq \emptyset$ e consequentemente, $H_{K}\left(f_{1}, \ldots, f_{m} ; g\right) \neq \emptyset$, ou seja, $K$ é hilbertiano.

O próximo passo é considerar a redução no número de variáveis.

Lema 3.4. Cada subconjunto de Hilbert de $K$ contém um subconjunto de Hilbert de $K$ da forma $H_{K}\left(f_{1}, \ldots, f_{m} ; g\right)$, onde para cada $i=1, \ldots, m, f_{i}$ é um polinômio em duas variáveis $T, X$ irredutível, isto é, $f_{i} \in K[T, X] \operatorname{com} g r_{X}\left(f_{i}\right) \geq 1$ e $0 \neq g \in K[X]$.

Dem.: Note que se tomarmos um subconjunto de Hilbert $H_{K}\left(f_{1}, \ldots, f_{m} ; g\right)$ de $K$ e considerarmos os subconjuntos $H_{i}=H_{K}\left(f_{i} ; g\right)$ temos que $H_{K}\left(f_{1}, \ldots, f_{m} ; g\right)=\bigcap_{i=1}^{m} H_{i}$. Logo, basta demonstrar o lema para os subconjuntos de Hilbert de $K$ da forma $H_{K}\left(f_{i} ; g\right)$, ou seja, devemos encontrar polinômios $f_{i j}$ irredutíveis em $K[T, X] \operatorname{com} g r_{X}\left(f_{i j}\right) \geq 1$, $j=1, \ldots, j(i)$ e $0 \neq g_{j} \in K[X]$ tais que $H_{K}\left(f_{i j}, \ldots, f_{i, j(i)} ; g_{j}\right) \subseteq H_{K}\left(f_{i} ; g\right)$. De fato, se tomarmos $h=g_{1} \cdots g_{m}$, e o conjunto $\left\{f_{i j} ; 1 \leq i \leq m\right.$ e $\left.1 \leq j \leq j(i)\right\} \subseteq K[T, X]$, teremos que $H_{K}\left(f_{i j} ; h\right)=\bigcap_{j} H_{K}\left(f_{i j} ; g_{j}\right) \subseteq \bigcap_{i} H_{K}\left(f_{i} ; g\right)=H_{K}\left(f_{1}, \ldots, f_{m} ; g\right)$.

Seja $H_{K}(f ; g)$ um subconjunto de Hilbert de $K$, onde $f \in K[T, \mathrm{X}]$ e $g \in K[T]$. Note que em $f$, pelo menos uma variável $X_{i}$ deve aparecer, pois caso contrário teríamos que $f(a, \mathrm{X})$ seria constante para todo $a \in K$, ou seja, o subconjunto de Hilbert $H_{K}(f ; g)$ seria vazio.

Seja $d>\max _{1 \leq j \leq n}\left\{g r_{X_{j}}(f)\right\}$. Aplicando a substituição de Kronecker $S_{d}$ a $f$ nas variáveis $X_{i}$ e considerando a fatoração de $S_{d}(f)$ em fatores irredutíveis sobre $K[T, Y]$, temos

$$
S_{d}(f)=\prod_{i \in I} h_{i}(T, Y)
$$


Tome $I=J \dot{U} J^{\prime}$ uma partição não trivial do conjunto de índices $I$. Da definição de $S_{d}$, temos que $S_{d}(f)$ tem grau no máximo $d^{n}$ na variável $Y$ e desde que a partição de $I$ é não trivial, temos que não pode ocorrer termos de ordem $d^{n}$ simultaneamente nos polinômios $\prod_{i \in J} h_{i}(T, Y)$ e $\prod_{i \in J^{\prime}} h_{i}(T, Y)$. Logo, o expoente de $Y$ que aparece nos polinômios $\prod_{i \in J} h_{i}(T, Y)$ e $\prod_{i \in J^{\prime}} h_{i}(T, Y)$ não excede $d^{n}-1$. Como $S_{d}$ é uma bijeção, existem polinômios $p_{J}, p_{J^{\prime}} \in K[T, \mathbf{X}]$ tais que $g r_{X_{j}}\left(p_{J}\right), g r_{X_{j}}\left(p_{J^{\prime}}\right)<d$, para $j=1, \ldots, n$ e

$$
S_{d}\left(p_{J}\right)=\prod_{i \in J} h_{i}(T, Y) \text { e } S_{d}\left(p_{J^{\prime}}\right)=\prod_{i \in J^{\prime}} h_{i}(T, Y)
$$

○ produto $p_{J}(T, \mathbf{X}) p_{J^{\prime}}(T, \mathbf{X})$ contém um monômio da forma $p_{J}^{\prime}(T, \mathbf{X})=$ $g_{J}(T) X_{1}^{\nu(1)} \cdots X_{n}^{\nu(n)}$ em que pelo menos um dos $\nu(j)$ é maior que $d-1$, pois caso contrário a relação $S_{d}(f)=S_{d}\left(p_{J}\right) S_{d}\left(p_{J^{\prime}}\right)=S_{d}\left(p_{J} p_{J^{\prime}}\right)$ implicaria numa fatoração não trivial do polinômio $f(T, \mathbf{X})$ em $p_{J}(T, \mathbf{X}) p_{J^{\prime}}(T, \mathbf{X})$.

Seja $g_{1}(T)$ o polinômio obtido do seguinte produto $\prod_{J} g(T) g_{J}(T) \in K[T]$ onde $J$ percorre todas as partições não triviais de $I$. Note que este produto é finito, pois $I$ é um conjunto finito. Vamos mostrar que $H_{K}\left(h_{i}, i \in I ; g_{1}\right) \subseteq H_{K}(f ; g)$. Seja $a \in H_{K}\left(h_{i}, i \in I ; g_{1}\right)$. Desde que $g_{1}(a) \neq 0$, resta mostrar que $f(a, \mathbf{X})$ é irredutível. Se $f(a, \mathbf{X})=q(\mathbf{X}) q^{\prime}(\mathbf{X})$ é uma fatoração não trivial de $f(a, \mathbf{X})$ em $K[\mathbf{X}]$, então (3.1) implica que

$$
\prod_{i \in I} h_{i}(a, Y)=S_{d}(f)(a, Y)=S_{d}(q)(Y) S_{d}\left(q^{\prime}\right)(Y)
$$

Usando a equação (3.2), temos que existe uma partição não trivial $J \dot{U} J^{\prime}$ de $I$ tal que $S_{d}(q)(Y)=\prod_{i \in J} h_{i}(a, Y)=S_{d}\left(p_{J}\right)(a, Y)$ e $S_{d}\left(q^{\prime}\right)(Y)=\prod_{i \in J^{\prime}} h_{i}(a, Y)=S_{d}\left(p_{J^{\prime}}\right)(a, Y)$. E desde que $S_{d}$ é bijetora, temos que $q(\mathbf{X})=p_{J}(\mathbf{X})$ e $q^{\prime}(\mathbf{X})=p_{J^{\prime}}(\mathbf{X})$ e portanto $p_{J}(a, \mathbf{X}) p_{J^{\prime}}(a, \mathbf{X})=f(a, \mathbf{X})$. Note que o primeiro membro possui um monômio $p_{J}^{\prime}(X, T)=$ $g_{J}(T) X_{1}^{\nu(1)} \cdots X_{n}^{\nu(n)}$ em que pelo menos um dos $\nu(j)$ é maior que $d-1$, enquanto $f(a, \mathbf{X})$ não possui tal monômio, o que é uma contradição. Portanto, $f(a, \mathbf{X})$ é irredutível em $K[\mathbf{X}]$.

O próximo resultado garante que podemos considerar somente os conjuntos de Hilbert de $K$ da forma $H_{K}\left(f_{1}, \ldots, f_{m}\right)$.

Proposição 3.5. Cada subconjunto de Hilbert de $K$ da forma $H_{K}\left(f_{1}^{\prime}, \ldots, f_{m}^{\prime} ; g\right)$ com $f_{i}^{\prime} \in K[T, X]$, para $i=1, \ldots, m$ irredutíveis e $g \neq 0 \in K[T]$ contém um conjunto de Hilbert da forma $H_{K}\left(f_{1}, \ldots, f_{m}\right)$ em que cada $f_{i}$ é um polinômio mônico e irredutível em $K[T, X]$ de grau positivo em $X$. Assim, se os conjuntos de Hilbert de $K$.da forma $H_{K}\left(f_{1}, \ldots, f_{m}\right)$ são não vazios, então $K$ é hilbertiano. Mais ainda, se $f_{1}^{\prime}, \ldots, f_{m}^{\prime}$ são polinômios separáveis, então $f_{1}, \ldots, f_{m}$ também são separáveis.

Dem.: Seja $H_{K}\left(f_{1}^{\prime}, \ldots, f_{m}^{\prime} ; g\right)$ um subconjunto de Hilbert de K. Então, do Lema 3.4 podemos assumir que $f_{i}^{\prime} \in K[T, X]$ são polinômios irredutível de grau positivo $n(i)$ em 
$X$, para todo $i=1, \ldots, m$ e $0 \neq g \in K[T]$. Note que podemos assumir $n(m) \geq 2$. De fato, se $n(i)=1$ para todo $1 \leq i \leq m$, então tomando $f_{m+1}^{\prime} \in K[T, X]$ irredutível, temos que $H_{K}\left(f_{1}^{\prime}, \ldots, f_{m+1}^{\prime} ; g\right) \subseteq H_{K}\left(f_{1}^{\prime}, \ldots, f_{m}^{\prime} ; g\right)$, e assim, basta fazer a demonstração para $H_{K}\left(f_{1}^{\prime}, \ldots, f_{m+1}^{\prime} ; g\right)$.

Denote o coeficiente dominante de $f_{i}^{\prime}$ por $c_{i}$, para todo $i=1, \ldots m$ e considere $g_{1}(T)=g(T) c_{1} \cdots c_{m}$. Note que $f_{i}^{\prime}(T, X)=c_{i} X^{n(i)} b_{i, n(i)}(T)+X^{n(i)-1} b_{i, n(i)-1}(T)+\cdots$ Tomando

$f_{i}^{\prime \prime}(T, X)=f_{i}^{\prime}(T, X) g_{1}(T)^{n(i)} c_{i}^{-1}=X^{n(i)} g_{1}(T)^{n(i)} b_{i, n(i)}(T)+c_{i}^{-1} X^{n(i)-1} g_{1}(T)^{n(i)} b_{i, n(i)-1}(T)+\cdots$ e substituindo $g_{1}(T) X$ por $Y$ temos o seguinte polinômio irredutível

$$
f_{i}(T, Y)=Y^{n(i)} b_{i, n(i)}+c_{i}^{-1} g_{1}(T) \sum_{j=0}^{n(i)-1} b_{i j}(T) Y^{j} .
$$

Vamos mostrar que $H_{K}\left(f_{1}, \ldots, f_{m}\right) \subseteq H_{K}\left(f_{1}^{\prime}, \ldots, f_{m}^{\prime} ; g\right)$. Seja $a \in H_{K}\left(f_{1}, \ldots, f_{m}\right)$, então $g_{1}(a) \neq 0$, pois caso contrário teríamos $f_{m}(a, Y)=Y^{n(m)} b_{i, n(m)}(a)$ que é redutível. Além disso, $a$ é tal que $f_{i}^{\prime}(a, X)$ é irredutível em $K[X]$. Portanto, $H_{K}\left(f_{1}, \ldots, f_{m}\right) \subseteq$ $H_{K}\left(f_{1}, \ldots, f_{m} ; g\right)$ e vê-se facilmente que se $f_{1}^{\prime}, \ldots, f_{m}^{\prime}$ são separáveis na variável $X$, então $f_{1}, \ldots, f_{m}$ também são separáveis.

Finalmente, considere um subconjunto de Hilbert de $K$. Usando o Lema 3.4 temos que este subconjunto contém um subconjunto da forma $H_{K}\left(f_{1}^{\prime}, \ldots, f_{m}^{\prime} ; g\right)$, com a notação acima. Assim, pela construção acima, concluimos que ele contém um subconjunto de Hilbert da forma $H_{K}\left(f_{1}, \ldots, f_{m}\right)$. Mas se os subconjuntos $H_{K}\left(f_{1}, \ldots, f_{m}\right)$ são não vazios, então o subconjunto de Hilbert original é também não vazio e assim, segue que $K$ é hilbertiano.

Finalmente, como um último resultado de redução, vamos mostrar que podemos reduzir o número de polinômios nos subconjuntos de Hilbert de $K$.

Teorema 3.6. Sejam $f_{1}, \ldots, f_{m} \in K[T, X]$ polinômios irredutíveis e separáveis na variável $X$ e seja $0 \neq g \in K[X]$. Então existe um polinômio $f \in K[T, X]$, separável e mônico na variável $X$ tal que $H_{K}(f) \subseteq H_{K}\left(f_{1}, \ldots, f_{m} ; g\right)$.

Dem.: Para cada $i=1, \ldots, m$, considere $x_{i}$ uma raiz de $f_{i}(T, X)$ em $K(T)_{s}$, o fecho separável de $K(T)$. Logo $K\left(T, x_{1}, \ldots, x_{m}\right)$ é uma extensão separável de $K(T)$. Então pelo teorema do elemento primitivo, temos que $K\left(T, x_{1}, \ldots, x_{m}\right)$ está contido em uma extensão separável $K(T, y)$ de $K(T)$ de grau $n \geq 1$. Note que $y$ pode ser tomado inteiro sobre $K[T]$. Seja $f^{\prime}(T, X)=\operatorname{irr}(y, K(T)) \in K(T) X$.

Como $g^{\prime}(a) \neq 0$, temos que $u_{i}\left(a, x_{i}\right) \neq 0$ e, conseqüentemente, $\operatorname{gr}\left(\operatorname{irr}\left(y, K\left(T, x_{i}\right)\right)\right)=$ $\operatorname{gr}\left(\operatorname{irr}\left(y, K\left(a, x_{i}\right)\right)\right)$. Assim $\left[K(T, y): K\left(T, x_{i}\right)\right]=\left[K(a, y): K\left(a, x_{i}\right)\right]$, para cada $i=$ $1, \ldots, m$.

Usando novamente que $g^{\prime}(a) \neq 0$, temos também que $r_{i}(a) \neq 0$, o que implica que $g r_{X}\left(f_{i}(T, X)\right)=g r_{X}\left(f_{i}(a, X)\right)$. Como $f_{i}(T, X)$ é irredutível e $x_{i}$ é uma raiz de $f_{i}(T, X)$, segue que $\left[K\left(T, x_{i}\right): K(T)\right]=g r_{X}\left(f_{i}(a, X)\right)$. 
Agora, do fato que $y$ é inteiro sobre $K[T]$ e $f^{\prime}(T, X)=\operatorname{irr}(y, K(T))$, temos que $f^{\prime}$ é mônico na variável $X$, o que implica que $g r_{X}\left(f^{\prime}(a, X)\right)=g r_{X}\left(f^{\prime}(T, X)\right)$ e, conseqüentemente, temos $[K(T, y): K(T)]=[K(a, y): K(a)]$.

Desde que

$$
[K(T, y): K(T)]=\left[K(T, y): K\left(T, x_{i}\right)\right]\left[K\left(T, x_{i}\right): K(T)\right]
$$

e

$$
[K(a, y): K(a)]=\left[K(a, y): K\left(a, x_{i}\right)\right]\left[K\left(a, x_{i}\right): K(a)\right],
$$

das igualdades obtidas acima, temos que $\left[K\left(T, x_{i}\right): K(T)\right]=\left[K\left(a, x_{i}\right): K(a)\right]$, para cada $i=1, \ldots, m$.

Se $f_{i}(a, X)$ é redutível para algum $i=1 \ldots, m$, então $\operatorname{gr}\left(\operatorname{irr}\left(x_{i}, K(a)\right)=\right.$ $\left[K\left(a, x_{i}\right): K(a)\right]<g r_{X}\left(f_{i}(a, X)\right)=\left[K\left(T, x_{i}\right): K(T)\right]$, o que é uma contradição.

Portanto, os polinômios $f_{1}(a, X), \ldots, f_{m}(a, X)$ são irredutíveis em $K[X]$ o que mostrar que $H_{K}\left(f^{\prime}, g^{\prime}\right) \subseteq H_{K}\left(f_{1}, \ldots, f_{m} ; g\right)$. Da Proposição 3.5 , temos que existe um polinômio $f \in K[T, X]$, separável e mônico na variável $X$ tal que $H_{K}(f) \subseteq H_{K}\left(f_{1}, \ldots, f_{m} ; g\right)$, como queríamos.

O subconjunto $H_{K}\left(f_{1}, \ldots, f_{m} ; g\right)$, onde os polinômios $f_{i} \in K[T, X]$ são separáveis e mônicos para todo $i=1, \ldots, m$ é chamado de subconjunto separável de Hilbert de $K$. Dizemos que um corpo $K$ é um de corpo Hilbertiano separável, se todos os seus subconjuntos separáveis de Hilbert da forma $H_{K}(f)$ são não vazios. Como conseqüencia do Teorema 3.6, temos

Teorema 3.7. Um corpo de característica zero é Hilbertiano se, e somente se é um corpo Hilbertiano separável.

Dem.: Seja $K$ um corpo Hilbertiano separável. Da Proposição 3.3, para verificar se $K$ é Hilbertiano, é suficiente considerar os subconjuntos de Hilbert de $K$ da forma $H_{K}\left(f_{1}, \ldots, f_{m} ; g\right), \operatorname{com} f_{1}, \ldots, f_{m} \in K[T, \mathbf{X}]$ e $0 \neq g \in K[T]$. Da Proposição 3.6 temos que dado um tal subconjunto de Hilbert, existe um polinômio $f \in K[T, X]$ irredutível, separável e mônico na variável $X$ tal que $H_{K}(f) \subseteq H_{K}\left(f_{1}, \ldots, f_{m} ; g\right)$. Desde que $K$ é Hilbertiano separável, temos que $H_{K}(f)$ é não vazio. Logo, $K$ é Hilbertiano.

A reciproca é imediata.

\subsection{Extensões separáveis de corpos Hilbertianos}

Nesta seção, mostraremos que extensões separáveis finitas de corpos Hilbertianos também são corpos Hilbertianos.

Antes de prosseguirmos vamos fazer uma observação a respeito da teoria de Galois. Se $K$ é um corpo, denotaremos por $\bar{K}$, o fêcho algébrico de $K$. Seja $K_{s}$ o fêcho separável de $K$ em $\vec{K}$, ou seja, $K_{s}=\{\alpha \in \bar{K} ; \alpha$ é separável sobre $K\}$. Ainda, denotaremos por 
$G(K)=G a l\left(K_{s} / K\right)$ o grupo de galois da extensão $K_{s} / K$, que é chamado de grupo de galois absoluto de $K$. Se $L / K$ é uma extensão finita e separável de grau $n$, então da teoria de Galois, sabemos que $[G(K): G a l(L / K)]=[L: K]=n$. Assim, existem $n$ classes laterias distintas no quociente $\frac{G(K)}{G a l(L / K)}$ que são os representantes de $G a l(L / K)$ em $G(K)$.

Lema 3.8. Seja $L$ uma extensão separável de um corpo infinito $K$ tal que $[L: K]=$ $d<\infty$ e sejam $\sigma_{1}, \ldots, \sigma_{d}$ os representantes de $G a l(L / K)$ em $G(K)$. Se $f \in L\left[X_{1}, \ldots, X_{n}\right]$ é um polinômio não constante, então existem $c_{1}, \ldots, c_{n} \in L$ tais que os $d$ polinômios $\sigma_{i}\left(f\left(X_{1}+c_{1}, \ldots, X_{n}+c_{n}\right)\right)$, para $i=1, \ldots d$, são relativamente primos em $\bar{K}\left[X_{1}, \ldots, X_{n}\right]$.

Dem.: Sejam $\alpha$ um elemento primitivo da extensão $L / K$ e $t_{i k}, i=1, \ldots d$ e $k=1, \ldots, n$, elementos algebricamente independentes sobre $K$. Denote por $\alpha_{i}$ o elemento $\sigma_{i}(\alpha)$ e para cada $i=1^{\prime} \ldots, d$ e $k=1, \ldots n$ considere

$$
u_{i k}=t_{i k}+\alpha_{i} t_{i k}+\cdots\left(\alpha_{i}\right)^{d-1} t_{i k}
$$

A aplicação $t \mapsto u$ é representada pela matriz

$$
A=\left(\begin{array}{ccccc}
1 & \alpha_{1} & \alpha_{1}^{2} & \cdots & \alpha_{1}^{d-1} \\
1 & \alpha_{2} & \alpha_{2}^{2} & \cdots & \alpha_{2}^{d-1} \\
\vdots & \ddots & \ddots & \ddots & \vdots \\
1 & \alpha_{d} & \alpha_{d}^{2} & \cdots & \alpha_{d}^{d-1}
\end{array}\right)
$$

Esta é uma matriz de Vandermond e seu determinante é $\prod_{i<j}\left(\sigma_{j}(\alpha)-\sigma_{i}(\alpha)\right) \neq 0$, ou seja, $A$ é inversível. Assim, o fato que $t_{i k}$ são algebricamente independentes implica que $u_{i k}$ são também algebricamente independentes. Considere $f=f_{1} \cdots f_{m}$ uma fatoração de $f$ como um produto de fatores irredutíveis em $\bar{K}\left[X_{1}, \ldots, X_{n}\right]$. Como os elementos $u_{i k}$ são algebricamente independentes, temos que $\left(\sigma_{i} f_{r}\right)\left(\mathbf{u}_{i}\right) \neq\left(\sigma_{j} f_{s}\right)\left(\mathbf{u}_{i}\right)$, para $i \neq j$ e $1 \leq r, s \leq m$, onde $u_{i}=\left(u_{i 1}, \ldots, u_{i n}\right)$. Portanto

$$
h(\mathbf{t})=\prod_{i<j} \prod_{r, s}\left(\left(\sigma_{i} f_{r}\right)\left(\mathbf{u}_{i}\right)-\left(\sigma_{j} f_{s}\right)\left(\mathbf{u}_{i}\right)\right) \neq 0
$$

Assim, existem $\mathbf{a}=\left(a_{i k}\right) \in M_{d \times n}(K)$ tal que $h(\mathbf{a}) \neq 0$. Para cada $k=1, \ldots, n$, seja

$$
c_{k}=a_{1 k}+\alpha a_{2 k}+\cdots+\alpha^{d-1} a_{d k} .
$$

Note que a aplicação $\mathrm{t} \rightarrow \mathrm{u}$ leva a em $\mathrm{v}=\left(v_{i j}\right)$, onde $v_{i j}=\sigma_{i}\left(c_{k}\right)$. Logo, para $\mathrm{c}=$ $\left(c_{1}, \ldots, c_{n}\right), \sigma_{i}\left(f_{r}(\mathbf{c})\right) \neq \sigma_{j}\left(f_{s}(\mathbf{c})\right)$ e portanto, $\sigma_{i}\left(f_{r}(\mathbf{X}+\mathbf{c})\right) \neq \sigma_{j}\left(f_{s}(\mathbf{X}+\mathbf{c})\right)$ para todos $1 \leq i<j \leq d$ e $1 \leq r, s \leq m$, pois $\sigma_{i}\left(f_{1}(\mathbf{X}+\mathbf{c})\right), \ldots \sigma_{i}\left(f_{m}(\mathbf{X}+\mathbf{c})\right)$ são as imagens dos fatores irredutíveis de $\sigma_{i}(f(\mathrm{X}+\mathrm{c}))$ em $\bar{K}\left[X_{1}, \ldots, X_{n}\right]$. Além disso, como $f_{1}, \ldots, f_{m}$ são irredutíveis em $\bar{K}\left[X_{1}, \ldots, X_{n}\right]$, temos que $\sigma_{i}\left(f_{1}(\mathbf{X}+\mathbf{c})\right), \ldots \sigma_{i}\left(f_{m}(\mathbf{X}+\mathbf{c})\right)$ são irredutíveis 
para todo $i=1, \ldots, d$. Portanto, se existe $g \in \bar{K}\left[X_{1}, \ldots, X_{n}\right]$ tal que $g$ divide $\sigma_{j}(f(\mathrm{X}+\mathrm{c}))$ e $g$ divide $\sigma_{i}(f(\mathbf{X}+\mathbf{c}))$ para $i \neq j$, então $g=\sigma_{i}\left(f_{r}(\mathbf{X}+\mathbf{c})\right)=\sigma_{j}\left(f_{s}(\mathbf{X}+\mathbf{c})\right)$ para algum $r, s$. Mas isso não é possível, desde que $\sigma_{i} \neq \sigma_{j}$. Assim, temos que $\sigma_{i}(f(\mathbf{X}+\mathbf{c}))$ são relativamente primos.

Proposição 3.9. Sejam $L / K$ uma extensão finita e separável e $f$ um polinômio irredutível em $L(\mathrm{~T})[\mathrm{X}]$. Então existe um polinômio irredutível $p \in K(\mathrm{~T})[\mathbf{X}]$ tal que $H_{K}(p) \subseteq H_{L}(f)$.

Dem.: Sejam $\sigma_{1}, \ldots, \sigma_{d}$ os representantes de $G a l(L / K)$ em $G(K)$. Vamos mostrar que $p=\prod_{i=1}^{d} \sigma_{i}(f)$ satisfaz o requerido. Note que podemos supor que polinômios $\sigma_{i}(f)$ são relativamente primos, pois usando o Lema 3.8 temos que existem elementos $c_{1}, \ldots, c_{n} \in$ $L(\mathrm{~T})$ tais que os polinômios $\sigma_{i}\left(f\left(\mathrm{~T}, X_{1}+c_{1}, \ldots, X_{n}+c_{n}\right)\right)$ são relativamente primos. Trocando $f$ por $\left.\left.f_{1}(\mathrm{~T}, \mathrm{X})\right)=f\left(\mathrm{~T}, X_{1}+c_{1}, \ldots, X_{n}+c_{n}\right)\right) \in L(\mathrm{~T}, \mathrm{X})$, temos que se $f_{1}(\mathrm{~T}, \mathrm{X})$ é irredutível, então $f\left(\mathrm{~T}, X_{1}+c_{1}, \ldots, X_{n}+c_{n}\right)$ também será, desde que a aplicação $\phi$ : $L(\mathrm{~T})[\mathrm{X}] \rightarrow L(\mathrm{~T})[\mathrm{X}]$ dada por $\phi(f(\mathrm{~T}, \mathrm{X}))=f(\mathrm{~T}, \mathrm{X}+\mathrm{c}))$ é um isomorfismo de anéis e $\phi(f)=f_{1}$.

Seja $\sigma \in G(K)$. Então $\sigma(p)=\sigma\left(\prod_{i=1}^{d} \sigma_{i}(f)\right)=\prod_{i=1}^{d} \sigma\left(\sigma_{i}(f)\right)=\prod_{i=1}^{d} \sigma_{i}(f)=p$, ou seja, os coeficientes de $p$ são fixados por $\sigma$. Logo, $p \in K(\mathrm{~T})[\mathrm{X}]$.

Mostremos agora que $p$ é irredutível. Seja $q \in K(\mathrm{~T})[\mathrm{X}]$ um fator irredutível $p$. Então em $L(\mathrm{~T})[\mathrm{X}]$, temos que $q$ é o produto de alguns $\sigma_{i}(f)$ e, como tais $\sigma_{i}(f)$ são irredutíveis em $L(\mathrm{~T})[\mathrm{X}]$, temos que existe $i_{0}$ tal que $\sigma_{i_{0}}(f)$ divide $q$ em $L(\mathrm{~T})[\mathrm{X}]$. Assim, para todo $j=1, \ldots, d$, temos que $\sigma_{j}\left(\sigma_{i_{0}}(f)\right)$ divide $\sigma_{j}(q)=q$, o que implica que $p$ divide $q$. Logo $p=q$ é irredutível em $K(\mathrm{~T})[\mathrm{X}]$.

Finalmente, mostremos que $H_{K}(p) \subseteq H_{L}(f)$. Seja, a $\in H_{K}(p)$. Então $p(\mathbf{a})(\mathbf{X})$ é não nulo e irredutível em $K[\mathrm{X}]$. Se existe uma decomposição não triviạl de $f(\mathbf{a})(\mathrm{X})=$ $g(\mathbf{X}) h(\mathbf{X})$ em $L[\mathbf{X}]$, então $p(\mathbf{a})(\mathbf{X})=\prod_{i=1}^{d} \sigma_{i}(f(\mathbf{a}, \mathbf{X}))=\prod_{i=1}^{d} \sigma_{i}(g(\mathbf{X})) \prod_{i=1}^{d} \sigma_{i}(h(\mathbf{X}))$ é uma decomposição não trivial de $p(\mathbf{a})(\mathbf{X})$ em $K[\mathbf{X}]$, o que é uma contradição. Logo, temos que $f(\mathbf{a})(\mathbf{X})$ é irredutível em $L[\mathbf{X}]$, ou seja, $H_{K}(p) \subseteq H_{L}(f)$.

Colorário 3.10. Seja L/K uma extensão finita e separável. Se $K$ é Hilbertiano, então $L$ também é hilbertiano.

Dem.: Usando a Proposição. 3.3, podemos considerar somente os subconjuntos de Hilbert de $L$ da forma $H_{L}\left(f_{1}^{\prime}, \ldots, f_{m}^{\prime} ; g\right)$, com $f_{1}^{\prime}, \ldots, f_{m}^{\prime} \in K[T, \mathrm{X}]$ e $0 \neq g \in K[T]$. Do Lema 3.4 e da Proposição 3.5 temos que existe um subconjunto de Hilbert de $L$ da forma $H_{L}\left(f_{1}, \ldots, f_{m}\right)$ tal que $H_{L}\left(f_{1}, \ldots, f_{m}\right) \subseteq H_{L}\left(f_{1}^{\prime}, \ldots, f_{m}^{\prime} ; g\right)$. Da proposição anterior, temos que existem polinômios $p_{i}$, para cada $i=1, \ldots, m$, tais que $H_{K^{\prime}}\left(p_{i}\right) \subseteq H_{L}\left(f_{i}\right)$. Logo, como $K$ é Hilbertiano, temos que $H_{K}\left(p_{1}, \ldots, p_{m}\right)=\cap_{i} H_{K}\left(p_{i}\right)$ é nāo vazio. Assim, como 
$\cap_{i} H_{K}\left(p_{i}\right) \subseteq \cap_{i} H_{L}\left(f_{i}\right)$, segue que $H_{L}\left(f_{1}, \ldots, f_{m}\right)$ é não vazio. Portanto, $H_{L}\left(f_{1}^{\prime}, \ldots, f_{m}^{\prime} ; g\right)$ é não vazio, como queríamos.

\subsection{Exemplos}

No restante deste capítulo, estaremos interessados em apresentar alguns exemplos de corpos Hilbertianos. Mostraremos que o corpo dos números racionais é Hilbertiano e, também que todo corpo de números algébricos são Hilbertianos. Inicialmente apresentaremos um lema cuja demonstração será omitida por ser bastante técnica e pode ser encontrada em [V].

Lema 3.11. Sejam $0<\rho \in \mathbb{Z}$ e $\varphi(T)=\sum_{\nu=k}^{-\infty} a_{\nu} T^{\frac{\nu}{n}}, a_{\nu} \in \mathbb{R}$ uma série de Laurent convergente para todo $t \geq \rho$. Suponha que $\varphi(T) \notin \mathbb{R}[T]$. Sejam $S_{\varphi}=\{t \in \mathbb{Z} ; t \geq \rho$ e $\varphi(t) \in \mathbb{Z}\}$ e $s_{\varphi}(N)=\#\left(S_{\varphi} \bigcap[1, N]\right)$, onde \# denota a cardinalidade do conjunto. Então existe $\delta \in \mathbb{R}$ com $0<\delta<1$ tal que $s_{\varphi}(N)=N^{\delta}$.

Como conseqüencia do próximo resultado teremos que o corpo dos números racionais é Hilbertiano.

Teorema 3.12. Seja $f(T, X) \in \mathbb{Z}[T, X]$ um polinômio irredutível em $\mathbb{Q}[T, X]$. Então existem infinitos valores de $t \in \mathbb{Z}$ tais que $f(t, X) \in \mathbb{Z}[X]$ e é irredutível em $\mathbb{Q}[X]$.

Dem.: Primeiramente, note que podemos supor que $f(T, X)$ é mônico na variável $X$. De fato, se $f(T, X)=a_{0}(T) X^{n}+a_{1}(T) X^{n-1}+\cdots a_{n}(T)$, então fazendo a mudança de variáveis $X \mapsto Y / a_{0}(T)$ temos que a imagem de $f(T, X)$ é da forma $\frac{g(T, Y)}{\left(a_{0}(T)\right)^{n-1}}$, onde $g(T, Y) \in \mathbb{Z}[T, Y]$ é mônico na variável $Y$. Note que para todo $t \in Z$ tall que $a_{0}(t) \neq 0$, temos que $g(t, Y)$ é irredutível em $\mathbb{Q}[Y]$ se, e somente se $f(t, X)$ é irredutível em $\mathbb{Q}[X]$.

Seja $f(T, X)=X^{n}+a_{1}(T) X^{n-1}+\cdots a_{n}(T) \in \mathbb{Z}[T, X]$ irredutível em $\mathbb{Q}[T, X]$. Sejam $\theta_{1}, \ldots, \theta_{n}$ todas as raízes de $f(T, X)$ no fêcho algébrico $\overline{\mathbb{Q}(T)}$ do corpo das funções racionais $\mathbb{Q}(T)$. Ainda, para cada $t \in \mathbb{Z}$, sejam $\theta_{1 t}, \ldots, \theta_{n t}$ todas as raízes do polinômio $f(t, X)$ no fêcho algébrico $\overline{\mathbb{Q}}$ de $\mathbb{Q}$. Como $\theta_{i t}$, para $i=1, \ldots, n$ são raízes de $f(t, X)$ e $f(T, X) \in$ $\mathbb{Z}[T, X]$, temos que são inteiras sobre $\mathbb{Z}$, ou seja, são raízes de um polinômio mônico com coeficientes em $\mathbb{Z}$.

Considere a seguinte aplicação $s_{t}: \mathbb{Q}[T] \rightarrow \mathbb{Q}$ dada por $s_{t}(p(T))=p(t)$. Esta aplicação se estende a um homomorfismo de anéis, que denotaremos ainda por $s_{t}: \mathbb{Q}\left[T, \theta_{1}, \ldots, \theta_{n}\right] \rightarrow \mathbb{Q}\left[T, \theta_{1 t}, \ldots, \theta_{n t}\right]$. Desde que $\left\{\theta_{1}, \ldots, \theta_{n}\right\}$ são todas as raízes de $f(T, X)$ em $\overline{\mathbb{Q}}(T)$ e, para cada $t \in \mathbb{Z},\left\{\theta_{1 t}, \ldots, \theta_{n t}\right\}$ são todas as raízes de $f(t, X)$ em $\mathbb{Q}$, temos que $s_{t}\left(\theta_{i}\right)=\theta_{i t}$, a menos de alguma permutação.

Por outro lado, se $h(T, X) \in \overline{\mathbb{Q}}(T)[X]$ é um fator de $f(T, X)$ então

$$
h(T, X)=\prod_{j=1}^{r}\left(X-\theta_{i_{j}}\right)=X^{r}+b_{r-1} X^{r-1}+\cdots+b_{0},
$$


onde cada $b_{i}$ é uma função simétrica elementar em $\theta_{i_{1}}, \ldots, \theta_{i_{r}}$. Além disso, como $f(T, X)$ é irredutível, segue que cada fator não trivial de $f$ em $\overline{\mathbb{Q}(T)}[X]$ terá pelo menos um coeficiente $b \in \overline{\mathbb{Q}(T)}$ tal que $b \notin \mathbb{Q}(T)$. Para cada fatoração não trivial de $f(T, X)$ em $\overline{\mathbb{Q}(T)}$ considere um coeficiente $b \in \overline{\mathbb{Q}(T)}$ tal que $b \notin \mathbb{Q}(T)$ e denote por $B$ o conjunto de todos elementos $b$. Note que $B$ é um conjunto finito, pois $f$ admite um número finito de fatorações.

Seja $t \in \mathbb{Z}$. Se $f(t, X)$ é redutível em $Q[X]$, então existe $b \in B$ tal que $s_{t}(b) \in \mathbb{Q}$, pois caso contrário, em cada fator não trivial de $f(t, X)$ em $\overline{\mathbb{Q}(T)}[X]$ sempre existe um coeficiente $b(t) \in \overline{\mathbb{Q}(T)}$ tal que $b(t) \notin \mathbb{Q}(T)$, o que contradiz o fato de $f(t, X)$ ser redutível em $\mathbb{Q}(T)$. Além disso, como $s_{t}(b) \in \mathbb{Q}\left[\theta_{1 t}, \ldots, \theta_{n t}\right]$ e os elementos $\theta_{i t}=s_{t}\left(\theta_{i}\right)$ são inteiros sobre $\mathbb{Z}$, segue que $s_{t}(b)$ é inteiro sobre $\mathbb{Z}$. Como $\mathbb{Z}$ é integralmente fechado, temos que $s_{t}(b) \in \mathbb{Z}$. Logo, se para todo $b \in B$ tivermos $s_{t}(b) \notin \mathbb{Z}$, então o polinômio $f(t, X)$ é irredutível em $\mathbb{Q}[X]$.

Dados $b \in B, \rho \in \mathbb{R}, 0<\rho$ e $N \in \mathbb{Z}$, consideremos $S_{b}=\left\{t \in \mathbb{Z} ; t>\rho\right.$ e $\left.s_{t}(b) \in \mathbb{Z}\right\}$ e $s(N)=\max _{b \in B}\left(\#\left(S_{b} \bigcap[1, N]\right)\right)$, onde $[1, N]$ é o intervalo dos inteiros entre 1 e $N$.

Usando a caracterização do fêcho algébico de $\mathbb{Q}(T)$ como séries de Laurent em $1 / T$ e resultados de geometria algébrica e análise, pode-se mostrar que $b=b(T)=\sum_{\nu=k}^{-\infty} a_{\nu} T^{\frac{\nu}{n}}$, com $a_{\nu} \in \mathbb{R}$ e satisfaz as hipóteses do Lema 3.11. Assim, existe $\delta \in \mathbb{R}$, com $0<\delta<1$ tal que $s(N)=N^{\delta}$.

Denote por $I$ o conjunto $\{t \in \mathbb{Z} ; \rho<t$ e $f(t, X)$ é irredutível sobre $Q[X]\}$. Note que se $t \in \mathbb{Z}, t>\rho$ e $t \notin S_{b}$ para todo $b \in B$, então $f(t, X)$ é irredutível em $\mathbb{Q}[X]$, ou seja, $\left(\bigcup_{b \in B} S_{b}\right)^{c} \subseteq I$, onde o complementar é tomado em $\{t \in \mathbb{Z} ; \rho<t\}$. Conseqüentemente, $\left(\bigcup_{b \in B} S_{b}\right)^{c} \cap[1, N] \subseteq I \cap[1, N]$.

$\operatorname{Mas}\left(\bigcup_{b \in B} S_{b}\right)^{c} \cap[1, N]=[1, N]-\left(\bigcup_{b \in B} S_{b}\right) \cap[1, N]=[1, N]-\bigcup_{b \in B}\left(S_{b} \cap[1, N]\right)$. Como $\#\left(S_{b} \bigcap[1, N]\right) \leq s(N) \leq c_{o} N^{\delta}$ para cada $b \in B$, temos que \# $\left(\bigcup_{b \in B}\left(S_{b} \cap[1, N]\right)\right) \leq$ $\#(B) c_{0} N^{\delta}$. Agora, \#(B) $c_{o}=c>0$, o que implica que $i(N)=\#(I \bigcap[1, N]) \geq \#([1, N]-$ $\left.\bigcup\left(S_{b} \cap[1, N]\right)\right) \geq N-c N^{\delta}=N\left(1-c N^{\delta-1}\right)$. Fazendo $N \rightarrow \infty$, segue que $c N^{\delta-1} \rightarrow 0$, pois $\delta-1<0$. Conseqüentemente $i(N) \rightarrow \infty$, ou seja, $i(N)$ não é limitado. Portanto, $I$ é um conjunto infinito, isto é, existem infinitos $t \in \mathbb{Z}$ tais que $f(t, X)$ é irredutível sobre $\mathbb{Q}[X]$.

Colorário 3.13. O corpo dos números racionais é Hilbertiano.

Dem.: Desde que $Q$ tem característica zero, da Proposição 3.7 basta mostrar que $\mathbb{Q}$ é Hilbertiano separável. Seja $f \in \mathbb{Q}[T, X]$ um polinômio irredutível. Sem perda da 
generalidade podemos supor que $f \in \mathbb{Z}[T, X]$. Logo, pelo teorema anterior, temos que $H_{\mathbb{Q}}(f) \cap \mathbb{Z} \neq \emptyset$, ou seja, $\mathbb{Q}$ é Hilbertiano separável, como queríamos.

Colorário 3.14. Se $K$ é um corpo de números algébricos, então $K$ é Hilbertiano.

Dem.: Segue diretamente do corolário acima e do Corolário 3.10. 


\section{Capítulo 4}

\section{Formas Traço Escalar sobre Corpos}

\section{Hilbertianos}

O nosso objetivo neste capítulo é mostrar que toda forma bilinear não degenerada sobre um corpo de números algébricos é isométrica a uma forma traço escalar.

Este resultado vale para uma família maior de corpos, a família dos corpos Hilbertianos de característica distinta de 2 e, do Corolário 3.14 temos que esta família contém a dos corpos de números algébricos. Assim, no que segue, neste e no próximo capítulo, todo corpo considerado será de característica distinta de 2. Apresentaremos então resultados sobre formas traço escalares sobre corpos Hilbertianos. Para tanto, iniciaremos com resultados sobre polinômios e matrizes sobre um corpo $K$.

Proposição 4.1. Sejam $K$ um corpo, $A \in M_{n}(K)$ uma matriz com polinômio característico irredutível e separável $f(X)=X^{n}+a_{1} X^{n-1}+\cdots+a_{n} \in K[X]$ e $L=K(x)=$ $\frac{K[X]}{(f(X))}$, onde $x=X+(f(X))$. Se $T: L \rightarrow L$ é a aplicação $K$-linear definida por $T(z)=x z$, para todo $z \in L$, então $A$ é a matriz de $T$ em relação a alguma base $\mathcal{B}=\left\{v_{1}, \ldots, v_{n}\right\}$ de $L$ sobre $K$.

Dem.: Considere a aplicação $L$-linear $T_{A}: L^{n} \rightarrow L^{n}$, definida por $T_{A}(z)=A z$, onde $z=\left(z_{1}, \ldots, z_{n}\right)^{t} \in L^{n}$. Como $f(x)=0$ e $f$ é o polinômio característico de $A$, então $x$ é um autovalor de $T_{A}$. Logo existe $v=\left(v_{1}, \ldots, v_{n}\right)^{t} \in L^{n}$, não nulo, tal que $A v=x v$, ou seja, $A v_{i}=x v_{i}$, onde $v_{i}=\left(0, \ldots, 0, v_{i}, 0, \ldots, 0\right)^{t}$ e $x v_{i}=\left(0, \ldots, 0, x v_{i}, 0, \ldots, 0\right)^{t}$.

Agora, se $\mathcal{B}=\left\{v_{1}, \ldots, v_{n}\right\}$ é uma base de $L$ sobre $K$, então escrevendo $z \in L$ como combinação linear dos elementos de $\mathcal{B}$, temos $z=\alpha_{1} v_{1}+\cdots+\alpha_{n} v_{n}$, com $\alpha_{i} \in K$. Então, $A z=A\left(\alpha_{1} v_{1}+\cdots+\alpha_{n} v_{n}\right)=\alpha_{1} A v_{1}+\cdots+\alpha_{n} A v_{n}=\alpha_{1} x v_{1}+\cdots+\alpha_{n} x v_{n}=$ $x\left(\alpha_{1} v_{1}+\cdots+\alpha_{n} v_{n}\right)=x z$, para todo $z \in L$ e, conseqüentemente $\mathcal{B}$ é a base de $L$ procurada.

Resta então mostrarmos que $\mathcal{B}$ é uma base de $L$ sobre $K$. Desde que $[L: K]=$ $g r(f)=n$, então é suficiente mostrarmos que $\mathcal{B}$ é linearmente independente. Para tanto, 
consideremos $N$ o corpo de raízes de $f(X)$ sobre $L$. Como $f(X) \in K[X]$ temos que $N$ é também o corpo de raizes de $f$ sobre $K$. Logo, $N=K\left(\beta_{1}, \ldots, \beta_{n}\right)$, onde $\beta_{1}, \ldots, \beta_{n}$ ' são todas as raízes de $f(X)$. Desde que $N$ é uma extensão normal finita e separável de $K$, temos que $N$ é uma extensão galoisiana de $K$. Seja $G=\operatorname{Gal}(N / K)$ o grupo de galois de $N$ sobre $K$. Como $L \subseteq N$, temos que existe um subgrupo $H \operatorname{de} G$, tal que $L=N^{H}$, ou seja, $H$ é o subgrupo dos automorfismos de $N$ que fixam os elementos de $L$. Além disso, $[G: H]=\operatorname{dim}_{K} L=n$. Sejam $\sigma_{1}, \ldots, \sigma_{n} \in G$ representantes das classes laterais de $H \mathrm{em}$ $G$, ou seja, $G=\bigcup_{1 \leq i \leq n}^{0} \sigma_{i} H$, onde $\sigma_{1}=i d$.

Consideremos a matriz $M=\left(\sigma_{i}\left(v_{j}\right)\right)=\left(\begin{array}{ccc}\sigma_{1}\left(v_{1}\right) & \cdots & \sigma_{1}\left(v_{n}\right) \\ \vdots & \ddots & \vdots \\ \sigma_{n}\left(v_{1}\right) & \cdots & \sigma_{n}\left(v_{n}\right)\end{array}\right) \in M_{n}(N)$.

Desde que $L=K(x)$ é separável, segue que $\sigma_{1}, \ldots, \sigma_{n} \in G$ são distintos. Além disso, $\sigma_{i}(x) \neq \sigma_{j}(x)$ para todo $i \neq j$, pois se existem índices $i \neq j$ tais que $\sigma_{i}(x)=\sigma_{j}(x)$, então para $y \in L$ temos que $y=a_{0}+a_{1} x+\cdots$ an $x^{n-1}$, pois $\left\{1, x, \ldots, x^{n-1}\right\}$ é base de $L$ sobre $K$. Logo, $\sigma_{i}(y)=\sigma_{i}\left(a_{0}+a_{1} x+\cdots a_{n} x^{n-1}\right)=a_{0}+a_{1} \sigma_{i}(x)+\cdots+a_{n} \sigma_{j}\left(x^{n-1}\right)=$ $a_{0}+a_{1} \sigma_{j}(x)+\cdots+a_{n} \sigma_{j}\left(x^{n-1}\right)=\sigma_{j}(y)$, ou seja, $\sigma_{i}(y)=\sigma_{j}(y)$, para todo $y \in L$, o que contradiz o fato das classes laterais serem distintas.

Ainda, as raízes de $f(X)$ são os conjugados de $x$, ou seja, são os elementos $\sigma_{i}(x)$ para $i=1, \ldots, n$. Desde que $A \in M_{n}(K)$, e $\left(v_{1}, \ldots, v_{n}\right)^{t}=\left(\sigma_{1}\left(v_{1}\right), \ldots, \sigma_{1}\left(v_{n}\right)\right)^{t}$ é um autovetor de $T_{A}$ associado ao autovalor $x$, temos que $\left(\sigma_{i}\left(v_{1}\right), \ldots, \sigma_{i}\left(v_{n}\right)\right)^{t}$ é autovetor de $T_{A}$ associado ao autovalor $\sigma_{i}(x)$, para cada $i=1, \ldots, n$, ou seja, as colunas de $M$ são autovetores de $T_{A}$. Como $f(X)$ é um polinômio separável, temos que os autovalores são distintos e, consequentemente, as colunas de $M$ são linearmente independentes, ou seja, $M$ é inversível. Desta maneira, $M$ define um isomorfismo $T_{M}: N^{n} \rightarrow N^{n}$, onde $T_{M}(y)=M y$, para todo $y=\left(y_{1}, \ldots, y_{n}\right)^{t} \in N^{n}$.

Se $\sum_{j=1}^{n} \lambda_{j} v_{j}=0$, com $\lambda_{j} \in K$, então $\sum_{j=1}^{n} \lambda_{j} \sigma_{i}\left(v_{j}\right)=0$, para todo $i=1 \cdots n$. Logo $M\left(\lambda_{1}, \cdots, \lambda_{n}\right)^{t}=(0, \cdots, 0)^{t}$. Multiplicando $M^{-1}$ à esquerda em ambos os lados obtemos $\left(\lambda_{1}, \cdots, \lambda_{n}\right)^{t}=(0, \cdots, 0)^{t}$, isto é, $\lambda_{j}=0$, para todo $j=1, \ldots, n$, o que mostra que $\mathcal{B}$ é linearmente independente sobre $K$, como queríamos.

Colorário 4.2. Sejam $A \in M_{n}(K), f(X) \in K[X]$ e $L=K(x)$ como na proposição anterior. Sejam $\varphi: L \rightarrow K$ uma aplicação $K$-linear qualquer e $b_{\varphi}$ a forma bilinear associada a $\varphi$, ou seja, $b_{\varphi}: L \times L \rightarrow K$ é definida por $b_{\varphi}(x, y)=\varphi(x y)$, para todo $x, y \in L$. Se $S$ é a matriz de $b_{\varphi}$ na base $\mathcal{B}$ encontrada na proposição anterior, então $S A=A^{t} S$.

Dem.: Da Proposição 4.1, temos que $A$ é a matriz da aplicação $K$-linear $T: L \rightarrow L$, definida por $T(z)=x z$, para todo $z \in L$, em relação à base $\mathcal{B}=\left\{v_{1}, \ldots, v_{n}\right\}$. Logo, a 
$j$-ésima coluna de $A=\left(a_{i j}\right)$ corresponde ao vetor das coordenadas de $T\left(v_{j}\right)$ com relação à base $\mathcal{B}$, ou seja, $T\left(v_{j}\right)=x v_{j}=\sum_{i=1}^{n} a_{i j} v_{i}$. Por hipótese, se $S=\left(s_{i j}\right)$, então $s_{i j}=b_{\varphi}\left(v_{i}, v_{j}\right)=$ $\varphi\left(v_{i} v_{j}\right)$ e, usando o fato que $\varphi$ é $K$-linear obtemos que a $i j$-ésima entrada da matriz $S A$, denotada por $(S A)_{i j}$ é

$$
(S A)_{i j}=\sum_{k=1}^{n} s_{i k} a_{k j}=\sum_{k=1}^{n} \varphi\left(v_{i} v_{k}\right) a_{k j}=\varphi\left(v_{i} \sum_{k=1}^{n} v_{k} a_{k j}\right)=\varphi\left(v_{i} x v_{j}\right) .
$$

Por outro lado, usando novamente que $\varphi$ é $K$-linear, temos:

$\left(A^{t} S\right)_{i j}=\sum_{k=1}^{n} a_{k i} \varphi\left(v_{k} v_{j}\right)=\varphi\left(\sum_{k=1}^{n} a_{k i} v_{k} v_{j}\right)=\varphi\left(x v_{i} v_{j}\right)$, para todo $i, j$, o que mostra que $S A=A^{t} S$, como queríamos.

Lema 4.3. Seja $S \in M_{n}(K)$ simétrica e inversível. Então $C \in M_{n}(K)$ é simétrica se, e somente se $A=C S$ satisfaz $A^{t} S=S A$.

Dem.: Se $C=C^{t}$, então $A^{t} S=(C S)^{t} S=S^{t} C^{t} S=S C S=S A$. Por outro lado, como $S C S=S A=A^{t} S=(C S)^{t} S=S^{t} C^{t} S=S C^{t} S$ e $S$ é inversível, obtemos que $C^{t}=C$.

O próximo resultado caracteriza quando uma matriz simétrica é a matriz de uma forma traço escalar.

Proposição 4.4. Seja $S \in M_{n}(K)$ uma matriz simétrica. Então existe $(L, b) \in \mathfrak{T} \mathfrak{T} E(K)$ tal que $S$ é a matriz de $b$ em relação a alguma base de $L$ sobre $K$ se, e somente se existe uma matriz simétrica $C$ tal que a matriz $C S$ tem polinômio característico irredutível e separável.

Dem.: Suponhamos que $S$ é a matriz de uma forma traço escalar não degenerada $(L, b) \in \mathfrak{T} \mathfrak{r}(K)$. Como a forma traço é não degenerada, a extensão $L / K$ é finita e separável (ver [L] pag. 284) e $S$ é inversível. Assim $L$ é uma extensão simples de $K$, ou seja $L=K(x)$. Seja $f(X)=X^{n}+a_{n-1} X^{n-1}+\cdots+a_{0} \in K[X]$ o polinômio irredutível e separável tal que $L=K(x) \cong \frac{K[X]}{(f(X))}$. Considerando a base $\mathcal{B}=\left\{1, x, \ldots, x^{n-1}\right\}$ de $L$ sobre $K$ e $T \in \operatorname{Hom}_{K}(L, L)$ definida por $T(z)=x z$, para todo $z \in L$. Temos que a matriz

$$
A=\left(\begin{array}{ccccc}
0 & 0 & \cdots & 0 & -a_{0} \\
1 & 0 & \cdots & 0 & -a_{1} \\
0 & 1 & \cdots & 0 & -a_{n-2} \\
\vdots & \vdots & \ddots & \vdots & \vdots \\
0 & 0 & \cdots & 1 & -a_{n-1}
\end{array}\right)
$$

é a matriz de $T$ com relação à base $\mathcal{B}$. Mais ainda, o polinômio característico de $A$ é $f(X)$. Do Corolário 4.2 temos que $S A=A^{t} S$. Tomando $C=A S^{-1}$, do Lema 4.3 temos que $C$ é simétrica e $C S=A$ tem polinômio característico irredutível e separável. 
Reciprocamente, sejam $A=C S \in M_{n}(K)$ com polinômio característico irredutível e separável $f(X)=X^{n}+a_{n-1} X^{n-1}+\cdots+a_{0} \in K[X]$ e $L=K(x) \cong \frac{K[X]}{(f(X))}$, onde $x=X+(f(X))$. Do Lema 4.3, temos que $C$ é simétrica se, e somente se $A^{t} S=S A$. Vamos, então encontrar as soluções de $A^{t} S=S A$. Da Proposição 4.1 temos que $A$ é a matriz da aplicação $T \in \operatorname{Hom}_{K}(L, L)$ definida por $T(z)=x z$, para todo $z \in L$, em relação a alguma base $\mathcal{B}=\left\{v_{1}, \ldots, v_{n}\right\}$ de $L$ sobre $K$.

Seja $\varphi \in \operatorname{Hom}_{K}(L, L)$. Considere a forma bilinear $b_{\varphi}: L \times L \rightarrow K$ associada à $\varphi$, ou seja, $b_{\varphi}(x, y)=\varphi(x y)$, para todo $x, y \in L$. Se $S$ é a matriz de $b_{\varphi}$ com relação à base $\mathcal{B}$, então do Corolário 4.2 temos que $A^{t} S=S A$. Logo, cada elemento de $\operatorname{Hom}_{K}(L, K)$ dá origem a uma solução de $A^{t} S=S A$. Vamos mostrar que essas são as únicas soluções, ou seja, as únicas soluções de $A^{t} S=S A$ são as matrizes associadas às formas $K$-lineares $\varphi \in \operatorname{Hom}_{K}(L, K)$.

Se $S_{0} \in M_{n}(K)$ é tal que $A^{t} S_{0}=S_{0} A$, então afirmamos que a matriz $S_{0}$ é determinada pela matriz $A$ e pela primeira coluna de $S_{0}$. De fato, tomando $v_{1}$ o primeiro elemento da base $\mathcal{B}$ e considerando que $\mathcal{B}^{\prime}=\left\{v_{1}, x v_{1}, x^{2} v_{1}, \ldots, x^{n-1} v_{1}\right\}$ é também uma base de $L$ sobre $K$, temos que para cada $r=0, \ldots, n-1$, o vetor $x^{r} v_{1}$ pode ser identificado com o vetor das suas coordenadas en relação à base $\mathcal{B}^{\prime}$, que é $(0, \ldots, 0,1,0, \ldots, 0)^{t}$, onde o elemento 1 aparece na $(r+1)$-ésima coordenada.

Vejamos agora como obter cada uma das colunas de $S_{0}$, a partir de $A$ e da primeira coluna de $S_{0}$. Usando a identificação acima, temos que a $(r+1)$-ésima coluna de $S_{0}$ é $S_{0}\left(x^{r} v_{1}\right)$. Desde que $A$ é a matriz da multiplicação por $x$ e $S_{0} A=A^{t} S_{0}$, para cada $r=1, \ldots, n-1$, temos

$$
S_{0}\left(x^{r} v_{1}\right)=S_{0}\left(A^{r} v_{1}\right)=\left(S_{0} A^{r}\right) v_{1}=\left(\left(A^{t}\right)^{r} S_{0}\right) v_{1}
$$

Portanto mostramos que o conjunto das soluções $S_{0} \in M_{n}(K)$ de $S_{0} A=A^{t} S_{0}$ está em correspondência biunívoca com um subconjunto de $M_{n \times 1}(K)$. Por outro lado, tínhamos que cada elemento de $\varphi \in \operatorname{Hom}_{K}(L, K)$ gerava uma solução. Como os espaços $\operatorname{Hom}_{K}(L, K)$ e $M_{n \times 1}(K)$ têm a mesma dimensão e portanto são isomorfos, do Corolário 4.2 concluimos que se $S$ é soluçao de $A^{t} S=S A$, então $S$ é a matriz de uma forma bilinear simétrica $b_{\varphi}: L \times L \rightarrow K$, associada a um elemento $\varphi \in \operatorname{Hom}_{K}(L, K)$. Mas do Lema 1.15, temos que cada elemento $\varphi \in \operatorname{Hom}_{K}(L, K)$ é do tipo $\operatorname{Tr}_{L / K}\langle 1\rangle(s$,$) , para algum s \in L$. Logo, a forma bilinear associada a $\varphi$ é do tipo $b_{\varphi}(x, y)=\operatorname{Tr}_{L / K}\langle s\rangle(x y)$, para todo $x, y \in L$, ou seja, é uma forma traço escalar.

Finalmente, resta mostrar que esta forma traço escalar é não degenerada, ou seja, $S$ é inversível. Desde que $A=C S$ tem polinômio característico irredutível, $f(X)=X^{n}+$ $a_{n-1} X^{n-1}+\cdots a_{0}$, temos que $A$ é inversível e como $\operatorname{det}(A)=\operatorname{det}(C S)=\operatorname{det}(C) \operatorname{det}(S)$, temos que $S$ é também inversível, corno queríamos.

Lema 4.5. Se $A=\left(X_{i j}\right)$ é uma matriz simétrica $n \times n$ onde $X_{i j}$ são indeterminadas, então $p\left(\left\{X_{i j}\right\}\right)=\operatorname{det}(A)$ é um polinômio irredutível em $K\left[\left\{X_{i j}\right\}\right]$. Além disso, $p\left(\left\{X_{i j}\right\}, X\right)=$ 
$\operatorname{det}\left(A-S^{-1}\left(X I_{n}\right)\right)$ é um polinômio irredutível em $K\left[\left\{X_{i j}\right\}, X\right]$, onde $S=\operatorname{diag}\left(s_{1}, \ldots, s_{n}\right)$ é uma matriz diagonal inversível e $I_{n}$ é a matriz identidade $n \times n$.

Dem.: Mostremos a primeira afirmação por indução sobre $n$. Se $n=1$, então $\operatorname{det}(A)=$ $X_{11}$ que é um polinômio irredutível em $K\left[X_{11}\right]$. Agora suponhamos que o resultado é verdadeiro para todas as matrizes simétricas de indeterminadas $k \times k, \operatorname{com} k \leq n-1$.

Escrevendo

$$
A=\left(\begin{array}{ccc|c}
X_{11} & \ldots & X_{1, n-1} & X_{1 n} \\
\vdots & \ddots & \vdots & \vdots \\
X_{1, n-1} & \ldots & X_{n-1, n-1} & \vdots \\
\hline X_{1 n} & \ldots & \ldots & X_{n n}
\end{array}\right)
$$

$G=\left\{\sigma \in S_{n} ; \sigma(n)=n\right\}$, onde $S_{n}$ é o grupo das permutações de $\{1, \ldots, n\}$, temos que

$$
\begin{aligned}
\operatorname{det}(A) & =\sum_{\sigma \in S_{n}} \operatorname{sign}(\sigma) X_{1, \sigma(1)} \ldots X_{n, \sigma(n)} \\
& =\sum_{\sigma \in G} \operatorname{sign}(\sigma) X_{1, \sigma(1)} \ldots X_{n-1, \sigma(n-1)} X_{n, n}+\sum_{\sigma \in \dot{S}_{n} \backslash G} \operatorname{sign}(\sigma) X_{1, \sigma(1)} \ldots X_{n, \sigma(n)} \\
& =X_{n n} \operatorname{det}\left(A^{\prime}\right)+\sum_{\sigma \in S_{n} \backslash G} \operatorname{sign}(\sigma) X_{1, \sigma(1)} \ldots X_{n, \sigma(n)},
\end{aligned}
$$

onde $A^{\prime}$ é a matriz $\left(X_{i j}\right) \operatorname{com} 1 \leq i, j \leq n-1$ e $\operatorname{sign}(\sigma)$ é o sinal da permutação $\sigma$, que é \pm 1 , de acordo com $\sigma$ ser par ou ímpar. Suponhamos que $\operatorname{det}(A)$ é redutível, ou seja, $\operatorname{det}(A)=X_{n n} \operatorname{det}\left(A^{\prime}\right)+\sum_{\sigma(n) \neq n} \operatorname{sign}(\sigma) X_{1, \sigma(1)} \ldots X_{n, \sigma(n)}=f g$, onde $f, g \in K\left[\left\{X_{i j}\right\}\right]$ são polinômios não constantes.

Tomando $X_{i j}^{\prime}=X_{i j}$ para $1 \leq i, j \leq n-1, X_{i j}^{\prime}=0$, para $j=n$ e $1 \leq i \leq n-1$, e $X_{n n}^{\prime}=$ 1 , temos que $f\left(\left\{X_{i j}^{\prime}\right\}\right) g\left(\left\{X_{i j}^{\prime}\right\}\right)=\operatorname{det}\left(A^{\prime}\right)$. Se $f\left(\left\{X_{i j}^{\prime}\right\}\right)$ e $g\left(\left\{X_{i j}^{\prime}\right\}\right)$ não são constantes, ternos um absurdo, pois $\operatorname{det}\left(A^{\prime}\right)$ é irredutível por hipótese de indução. Suponhamos então, sem perda da generalidade que $f\left(\left\{X_{i j}^{\prime}\right\}\right)=\lambda$, com $\lambda \in K$. Assim, temos que $g\left(\left\{X_{i j}^{\prime}\right\}\right)=\lambda^{-1} \operatorname{det}\left(A^{\prime}\right)$. Podemos então escrever $f=\lambda X_{n n}+\sum_{k=1}^{n} X_{k n} f_{k}$ e $g=\lambda^{-1} \operatorname{det}\left(A^{\prime}\right)+$ $\sum_{k=1}^{n} X_{k n} g_{k}$. Conseqüentemente, $f g=X_{n n} \operatorname{det}\left(A^{\prime}\right)+\sum_{k=1}^{n} X_{k n} h_{k}=\operatorname{det}(A)$. Daí $g r(f g) \geq n+1$ e $g r(\operatorname{det}(A))=n$, o que é um absurdo. Portanto, $\operatorname{det}(A)$ é irredutível.

Além disso, temos que $p\left(\left\{X_{i j}\right\}\right)=\operatorname{det}(A)$ continua irredutível em $K_{1}=K\left[\left\{X_{i j}, X\right\}\right]$, onde $X$ é uma nova indeterminada. Desde que $S=\operatorname{diag}\left(s_{1}, \ldots, s_{n}\right)$ é inversível a aplicação $K$-linear $\phi: K_{1} \rightarrow K_{1}$, definida por:

$$
\begin{aligned}
\phi(X) & =X \\
\phi\left(X_{i j}\right) & =X_{i j}, \text { para } i \neq j \\
\phi\left(X_{i i}\right) & =X_{i i}-s_{i}^{-1} X,
\end{aligned}
$$


é um $K$-automorfismo de $K_{1}$, pois os elementos $\left\{X_{i j}, i \neq j\right\} \bigcup\left\{X_{i i}-s_{i}^{-1} X\right\} \bigcup\{X\}$ geram $K_{1}$, como $K$-álgebra. Portanto,

$$
p\left(X_{i j}, X\right)=\operatorname{det}\left(\begin{array}{cccc}
X_{11}-s_{1}^{-1} X & X_{12} & \ldots & X_{1 n} \\
X_{21} & X_{22}-s_{2}^{-1} X & \ldots & X_{2 n} \\
\vdots & \ddots & \ddots & \vdots \\
X_{1 n} & X_{2 n} & \ldots & X_{n n}-s_{n}^{-1} X
\end{array}\right)=\operatorname{det}(\phi(A))
$$

é um polinômio irredutível em $K_{1}$, pois é a imagem de um polinômio irredutível por um $K$-automorfismo.

Agora estamos aptos a apresentar o principal resultado deste capítulo.

Teorema 4.6. Seja $K$ um corpo hilbertiano com $\operatorname{car}(K) \neq 2$. Então $\mathfrak{B i l}(K)=\mathfrak{T r E}(K)$, ou seja, toda forma bilinear não degenerada sobre $K$ é isométrica a uma forma traço escalar.

Dem.: Seja $(V, b) \in \operatorname{Bil}(K)$. Desde que $K$ tem $\operatorname{car}(K) \neq 2$, segue do Lema 1.5 que $(V, b)$ admite uma base ortogonal, ou seja, existe uma base de $V$ sobre $K$ tal que a matriz de $b$ em relação à esta base é uma matriz diagonal $S=\operatorname{diag}\left(s_{1}, \ldots, s_{n}\right)$. Seja $A=\left(X_{i j}\right)$ uma matriz simétrica, onde $X_{i j}$ são indeterminadas.

Do Lema 4.5 temos que $p\left(\left\{X_{i j}\right\}, X\right)=\operatorname{det}\left(A-S^{-1}\left(X I_{n}\right)\right)$ é um polinômio irredutível em $K\left[\left\{X_{i j}\right\}, X\right]$. Seja $h\left(\left\{X_{i j}\right\}, X\right)=\prod_{i=1}^{n}\left(X_{i i}-s_{i}^{-1} X\right) \in K\left[\left\{X_{i j}\right\}, X\right]$ o produto da diagonal principal de $\left(A-S^{-1}\left(X I_{n}\right)\right)$. Considerando $h\left(\left\{X_{i j}\right\}, X\right)$ como um polinômio em $K\left(\left\{X_{i j}\right\}\right)[X]$ temos que as raízes de $h\left(\left\{X_{i j}\right\}, X\right)$ são $s_{i} X_{i i}$, para $i=1, \ldots, n$ que são todas distintas, ou seja, $h\left(\left\{X_{i j}\right\}, X\right)$ é um polinômio separável na variável $X$. Assim, $\frac{\partial h}{\partial X}\left(\left\{X_{i j}\right\}, x\right) \neq 0$, onde $x=X+\left(h\left(\left\{X_{i j}\right\}, X\right)\right)$ em $\frac{K\left(\left\{X_{i j}\right\}\right)[X]}{\left(h\left(\left\{X_{i j}\right\}, X\right)\right)}$, pois $x$ é uma raiz de $h\left(\left\{X_{i j}\right\}, X\right)$. Logo, temos que $\frac{\partial h}{\partial X}\left(X_{i j}, X\right)$ é um polinômio não nulo em $K\left(\left\{X_{i j}\right\}\right)[X]$. Escrevendo $p\left(\left\{X_{i j}\right\}, X\right)=h\left(\left\{X_{i j}\right\}, X\right)+\sum_{l=0}^{n-1} f_{l}\left(\left\{X_{i j}\right\}\right) X^{l} \in K\left[\left\{X_{i j}\right\}, X\right]$, temos que

$$
\frac{\partial p}{\partial X}\left(\left\{X_{i j}\right\}, X\right)=\frac{\partial h}{\partial X}\left(\left\{X_{i j}\right\}, X\right)+\sum_{l=1}^{n-1} l f_{l}\left(\left\{X_{i j}\right\}\right) X^{l-1}
$$

Por outro lado, usando o fato que $p\left(\left\{X_{i j}\right\}, X\right)=\operatorname{det}\left(A-S^{-1}\left(X I_{n}\right)\right)$ temos que para cada $l=0, \ldots, n-1$ existem $r, s \in\{1, \ldots, n\}$ distintos tais que $f_{l}\left(\left\{X_{i j}\right\}\right)$ é um múltiplo de $X_{r s}$, pois cada $f_{l}\left(\left\{X_{i j}\right\}\right)$ contém uma indeterminada que não está na diagonal principal. Concluímos assim que $\frac{\partial p}{\partial X}\left(\left\{X_{i j}\right\}, X\right) \neq 0$, pois caso contrário, tomando $X_{i j}=0$, para 
$i \neq j$, obtemos $\frac{\partial h}{\partial X}\left(\left\{X_{i j}\right\}, X\right)=0$, o que é uma contradição. Note que $\frac{\partial p}{\partial X}\left(\left\{X_{i j}\right\}, X\right) \neq$ 0 em $K\left(\left\{X_{i j}\right\}\right)[X]$ e como $K\left(\left\{X_{i j}\right\}\right)$ é um corpo, temos que $\frac{\partial p}{\partial X}\left(\left\{X_{i j}\right\}, X\right)$, como um elemento de $K\left(\left\{X_{i j}\right\}\right)[X]$, possui no máximo $n-1$ raízes. Mas, como $K$ é um corpo Hilbertiano, temos da Proposição 3.1 que $K$ é infinito. Logo existe $\alpha \in K$ tal que $\frac{\partial p}{\partial X}\left(\left\{X_{i j}\right\}, \alpha\right) \neq 0$

Considere o seguinte subconjunto de Hilbert de $K$,

$H_{K}\left(p ; \frac{\partial p}{\partial X}\right)=\left\{\left(\lambda_{i j}\right) \in M_{n}(K) ; p\left(\left\{\lambda_{i j}\right\}, X\right)\right.$ é irredutível em $K[X]$ e $\left.\frac{\partial p}{\partial X}\left(\left\{\lambda_{i j}\right\}, \alpha\right) \neq 0\right\}$

Como $K$ é Hilbertiano, temos que $H_{K}\left(p ; \frac{\partial p}{\partial X}\right) \neq \emptyset$, ou seja, existe uma matriz simétrica $C=\left(\lambda_{i j}\right) \in M_{n}(K)$ tal que $p\left(\left\{\lambda_{i j}\right\}, X\right)$ é irredutível e $\frac{\partial p}{\partial X}\left(\left\{\lambda_{i j}\right\}, \alpha\right) \neq 0$, ou seja, $\frac{\partial p}{\partial X}\left(\left\{\lambda_{i j}\right\}, X\right)$ é um polinômio não nulo em $K[X]$. Agora, desde que $p\left(\left\{\lambda_{i j}\right\}, X\right)$ é irredutível e tem grau maior que o grau de $\frac{\partial p}{\partial X}\left(\left\{\lambda_{i j}\right\}, X\right)$, temos que $\operatorname{mdc}\left(p\left(\left\{\lambda_{i j}\right\}, X\right), \frac{\partial p}{\partial X}\left(\left\{\lambda_{i j}\right\}, X\right)\right)=1$, ou seja, $p\left(\lambda_{i j}, X\right)$ é um polinômio separável em $X$.

Note que

$$
\operatorname{det}\left(X I-\left(\lambda_{i j}\right) S\right)\left(\operatorname{det}\left(S^{-1}\right)\right)=\operatorname{det}\left(X S^{-1}-\left(\lambda_{i j}\right)\right)= \pm(\operatorname{det}(S)) p\left(\left\{X_{i j}\right\}, X\right) .
$$

Assim, obtemos uma matriz simétrica $C=\left(\lambda_{i j}\right) \in M_{n}(K)$ para a qual $C S$ tem polinômio irredutível e separável sobre $K$. Portanto, o teorema segue da Proposiçāo 4.4.

Colorário 4.7. Seja $K$ um corpo de números algébricos. Toda forma bilinear simétrica não degenerada sobre $K$ é isométrica a uma forma traço escalar.

Dem.: Segue diretamente do Teorema 4.6 e do Corolário 3.14 . 


\section{Capítulo 5}

\section{Formas Traço sobre Corpos de}

\section{Números Algébricos}

O Teorema 4.6 nos diz que formas bilineares não degeneradas sobre corpos Hilbertianos de característica distinta de 2 são isométricas a formas traço escalares. Em se tratando de corpos Hilbertianos ordenados, este resultado pode ser refinado, podemos mostrar que formas bilineares positivas, ou seja, que têm assinatura positiva para todas as ordens de $K$, são algébricas.

Antes de trabalharmos diretamente com os corpos hilbertianos, vamos apresentar alguns resultados envolvendo forma traço.

Lema 5.1. Sejam $K$ um corpo, $f(X)=X^{5}+\alpha X+\lambda \in K[X]$ um polinômio irredutível e $L=\frac{K[X]}{(f(X))}$. Seja $x \in L$ uma raiz de $f(X)$. Então:

(i) $\operatorname{tr}_{L / K}(1)=5$;

(ii) $\operatorname{tr}_{L / K}(x)=\operatorname{tr}_{L / K}\left(x^{2}\right)=\operatorname{tr}_{L / K}\left(x^{3}\right)=\operatorname{tr}_{L / K}\left(x^{6}\right)=\operatorname{tr}_{L / K}\left(x^{7}\right)=0$;

(iii) $\operatorname{tr}_{L / K}\left(x^{4}\right)=-4 \alpha$;

(iv) $\operatorname{tr}_{L / K}\left(x^{5}\right)=-5 \lambda$;

(v) $\operatorname{tr}_{L / K}\left(x^{8}\right)=4 \alpha^{2}$.

Dem.: Sejam $\lambda_{1}, \ldots, \lambda_{5}$ todas as raízes de $f(X)$ no fêcho algébrico $\bar{K}$ de $K$. Seja $\operatorname{tr}_{t: K}=$ $\sigma_{1}+\cdots+\sigma_{5}$, onde $\sigma_{i}$ são as imersões de $L$ em $\bar{K}$. Assim, como $x$ é uma raiz de $f(X)$, então $\lambda_{i}=\sigma_{i}(x), \quad i=1, \ldots, 5$ são todas as raízes de $f(X)$. Logo, temos que $\operatorname{tr}_{L / K}\left(x^{i}\right)=$ $\sigma_{1}\left(x^{i}\right)+\cdots+\sigma_{5}\left(x^{i}\right)=\sigma_{1}(x)^{i}+\cdots+\sigma_{5}(x)^{i}=\lambda_{1}^{i}+\cdots+\lambda_{5}^{i}$ são funções simétricas nas raízes de $f(X)$, para cada $i=1, \ldots, 5$. Além disso, em $\bar{K}[X]$ temos a seguinte fatoração de $f(X)$ : 


$$
f(X)=\left(X-\lambda_{1}\right) \cdots\left(X-\lambda_{5}\right)=X^{5}-\left(\lambda_{1}+\cdots+\lambda_{5}\right) X^{4}+\left(\lambda_{1} \lambda_{2}+\lambda_{1} \lambda_{3}+\lambda_{1} \lambda_{4}+\cdots+\right.
$$
$\left.\lambda_{4} \lambda_{5}\right) X^{3}-\left(\lambda_{1} \lambda_{2} \lambda_{3}+\lambda_{1} \lambda_{2} \lambda_{4}+\cdots+\lambda_{3} \lambda_{4} \lambda_{5}\right) X^{2}+\left(\lambda_{1} \lambda_{2} \lambda_{3} \lambda_{4}+\lambda_{1} \lambda_{2} \lambda_{3} \lambda_{5}+\cdots \lambda_{2} \lambda_{3} \lambda_{4} \lambda_{5}\right) X-$ $\lambda_{1} \lambda_{2} \lambda_{3} \lambda_{4} \lambda_{5}$

Portanto, podemos escrever $t r_{L / K}$ em função dos coeficientes de $f(X)$, que são as funções simétricas elementares das raízes de $f(X)$, ou seja, usando a igualdade acima obtemos $\operatorname{tr}_{L / K}(1)=\sigma_{1}(1)+\cdots+\sigma_{5}(1)=1+\cdots+1=5, \operatorname{tr}_{L / K}(x)=\lambda_{1}+\cdots+\lambda_{5}=0$ e $\operatorname{tr}_{L / K}\left(x^{2}\right)=\lambda_{1}^{2}+\cdots+\lambda_{5}^{2}$ é simétrico de grau 2 e, portanto, pode ser escrito como combinação das funções simétricas elementares de graus 1 e 2 , a saber, os coeficientes de $X^{\frac{1}{4}}$ e $X^{3}$ de $f(X)$. Desde que estes coeficientes são nulos, temos que $\operatorname{tr}_{L / K}\left(x^{2}\right)=0$.

Para $x^{3}$ temos $\operatorname{tr}_{L / K}\left(x^{3}\right)=\lambda_{1}^{3}+\cdots+\lambda_{5}^{3}$ que pode ser escrito em função dos coeficientes de $X^{4}, X^{-3}$ e $X^{2}$ de $f(X)$, que são todos nulos. Logo $\operatorname{tr}_{L / K}\left(x^{3}\right)=0$.

Agora, desde que $f(X)$ é irredutível, temos que $\lambda \in K$ é não nulo. Logo, temos que $g(X)=X^{5}+\alpha \lambda^{-1} X^{4}+\lambda^{-1}$ é irredutível em $K[X]$. Note que $g\left(x^{-1}\right)=x_{-}^{-5}+$ $\alpha \lambda^{-1} x^{-4}+\lambda^{-1}=\lambda^{-1} x^{-5} f(x)=0$. Portanto, considerando os coeficientes de $g(X)$, temos que $\operatorname{tr}_{L / K}\left(x^{-1}\right)=-\alpha \lambda^{-1}$. Mas, $x^{5}+\alpha x+\lambda=0$ e assim $x^{4}+\alpha+\lambda x^{-1}=0$, o que implica que $\operatorname{tr}_{L / K}\left(x^{4}\right)=-\alpha \operatorname{tr}_{L / K}(1)-\lambda \operatorname{tr}_{L / K}\left(x^{-1}\right)=-5 \alpha+\alpha=-4 \alpha$. Para $i=5$, temos $\operatorname{tr}_{L / K}\left(x^{5}\right)=-\operatorname{tr}_{L / K}(\lambda)-\alpha \operatorname{tr}_{L / K}(x)=-5 \lambda$.

Note ainda que para $i \geq 5, x^{i}=x^{5+(i-5)}$, ou melhor, $x^{i}=x^{i-5} x^{5}=t^{i-5}(-\lambda-\alpha x)=$ $-\alpha x^{i-5+1}-\lambda x^{i-5}$. Portanto, $\operatorname{tr}_{L / K}\left(x^{6}\right)=-\alpha \operatorname{tr}_{L / K}\left(x^{2}\right)-\lambda \operatorname{tr}_{L / K}(x)=0, \operatorname{tr}_{L / K}\left(x^{7}\right)=$ $-\operatorname{atr}_{L / K}\left(x^{3}\right)-\lambda \operatorname{tr}_{L / K}\left(x^{2}\right)=0$ e finalmente, $\operatorname{tr}_{L / K}\left(x^{8}\right)=-\alpha \operatorname{tr}_{L / K}\left(x^{4}\right)-\lambda \operatorname{tr}_{L / K}\left(x^{3}\right)=$ $-\alpha(-4 \alpha)=4 \alpha^{2}$, como queríamos.

Lema 5.2. Sejam $K$ um corpo $\operatorname{com} \operatorname{car}(K) \neq 5, f(X)=X^{5}+\alpha X+\alpha \beta$ um polinômio irredutível sobre $K$ e $L=\frac{K[X]}{(f(X))}$. Então $\operatorname{Tr}_{L / K}\langle 1\rangle=\langle 1,1,-\delta\rangle$ em $W(K)$, onde $\delta=$ $a^{4}\left(4^{4} \alpha+5^{5} \beta^{4}\right)$ é o determinante de $\operatorname{Tr}_{L / K}\langle 1\rangle$.

Dem.: Seja $\lambda=\alpha \beta$. Então $f(X)=X^{5}+\alpha X+\lambda$. Sejam $x$ uma raiz de $f(X)$ em $L$ e $\mathcal{B}=\left\{1, x, x^{2}, x^{3}, x^{4}\right\}$ uma base de $L$ sobre $K$. Usando o lema anterior temos que a matriz da forma traço de $L$ sobre $K, \operatorname{Tr}_{L / K}\langle 1\rangle$, com relação à base $\mathcal{B}$ é:

$$
M=\left(\begin{array}{ccccc}
5 & 0 & 0 & 0 & -4 \alpha \\
0 & 0 & 0 & -4 \alpha & -5 \lambda \\
0 & 0 & -4 \alpha & -5 \lambda & 0 \\
0 & -4 \alpha & -5 \lambda & 0 & 0 \\
-4 \alpha & -5 \lambda & 0 & 0 & 4 \alpha^{2}
\end{array}\right) .
$$

Considerando o subespaço $U$ de $L$ gerado por $\left\{1, x^{2}\right\}$ temos que a forma traço restrita à $U$, com relação à base $\left\{1, x^{2}\right\}$ é $\langle 5,-4 \alpha\rangle \simeq\langle 5,-\alpha\rangle$. Como $\operatorname{car}(K) \neq 2$ e 5 , temos que $\left(U, \operatorname{Tr}_{L / K}\langle 1\rangle\right)$ é um subespaço não degenerado de $\left(L, \operatorname{Tr}_{L / K}\langle 1\rangle\right)$. Então de Lema 1.2 temos que $L=U \perp U^{\perp}$. 
Desde que $\operatorname{Tr}_{L / K}\langle 1\rangle(1, x)=\operatorname{tr}_{L / K}(x)=0, \operatorname{Tr}_{L / K}\langle 1\rangle(x, x)=\operatorname{tr}_{L / K}\left(x^{2}\right)=0 \mathrm{e}$ $\operatorname{Tr}_{L / K}\langle 1\rangle\left(x^{2}, x\right)=\operatorname{tr}_{L / K}\left(x^{3}\right)=0$, temos que $x \in U^{\perp}$ e é um vetor isotrópico. Da Proposição 1.7, temos que $\left(\operatorname{Tr}_{L / K}\langle 1\rangle\right)_{U^{\perp}}=\langle 1,-1, \beta\rangle$, para algum $\beta \in \dot{K}$. Portanto, $\operatorname{Tr}_{L / K}\langle 1\rangle \simeq\langle 5,-\alpha, 1,-1, \beta\rangle$ e, $\operatorname{des} d e$ que $\operatorname{det}(M)=\delta=\left(4^{4} \alpha^{5}+5^{5} \lambda^{4}\right)$ módulo $\dot{K}^{2}$, temos que $\operatorname{Tr}_{L / K}\langle 1\rangle \simeq\langle 5,-\alpha, 1,-1,5 \alpha \delta\rangle$, ou seja $\operatorname{Tr}_{L / K}\langle 1\rangle=\langle 5,-\alpha, 5 \alpha \delta\rangle$ em $W(K)$. Note que $\delta=\left(4^{4} \alpha^{4}\right) \alpha+\left(5^{4} \lambda^{4}\right) 5$, isto é, a forma bilinear $\langle 5, \alpha\rangle$ representa $\delta$. Assim, de [Lam] pag.20, temos que $\langle 5, \alpha\rangle \simeq\langle\delta, 5 \alpha \delta\rangle$.

Então, em $W(K)$ temos que $\langle 5,-\alpha, 5 \alpha \delta\rangle=\langle 5,-\alpha, 5, \alpha,-\delta\rangle$ pois $\langle 5,-\alpha, 5 \alpha \delta, \delta\rangle \simeq$ $\langle 5,-\alpha, 5, \alpha\rangle$. Conseqüentemente $\operatorname{Tr}_{L / K}\langle 1\rangle=\langle 5,5,-\delta\rangle$ em $W(K)$. Note ainda que $\langle 5,5\rangle \simeq$ $\langle 1,1\rangle$, pois $5=1^{2}+2^{2}$, ou seja, a forma $\langle 1,1\rangle$ representa 5. Portanto, $\operatorname{Tr}_{L / K}\langle 1\rangle=$ $\langle 5,5,-\delta\rangle=\langle 1,1,-\delta\rangle$ em $W(K)$, como queríamos mostrar.

Como conseqüencia deste último resultado, podemos mostrar que certos elementos em $W(K)$ são algébricos.

Colorário 5.3. Seja $K$ um corpo Hilbertiano com $\operatorname{car}(K) \neq 5$. Então os elementos de $W(K)$ representados por espaços bilineares do tipo $\langle 1,1,-\delta\rangle$, com $\delta \in \dot{K}$ são algébricos.

Dem.: Dado $\delta \in \dot{K}$, desde que $\operatorname{car}(K) \neq 2$, tomando $\alpha(Y)=4^{-4}\left(\delta-5^{5} Y^{4}\right)$, onde $Y$ é uma indeterminada, temos que $f(X, Y)=X^{5}+\alpha(Y) X+\alpha(Y) Y$ tem discriminante $\delta$. Note que $\alpha^{\prime}(Y)=-4^{-3} 5^{5} Y^{3}$, que tem todas as raízes iguais a zero. Desde que $\alpha(0) \neq 0$, segue que $\alpha(Y)$ é um polinômio separável de $K[Y]$. Seja $p(Y)$ um fator irredutível de $\alpha(Y)$, então $p(Y) \mid \alpha(Y)$ e $p^{2}(Y) \nmid \alpha(Y)$. Logo $f(X, Y)$ é irredutível em $K(Y)[X]$ pelo critério de irredutibilidade de Eisenstein. Como $K$ é Hilbertiano, podemos escolher $\beta \in K$ tal que $f(\beta, X)$ continua irredutível em $K[X]$. Agora, $f(\beta, X)=X^{5}+\alpha X+\alpha \beta$ é como no Lema 5.2 e o resultado segue.

O nosso próximo passo é mostrar que sobre um corpo Hilbertiano ordenado, todos os elementos do anel de Witt representados por um espaço bilinear de dimensão $1,\langle\alpha\rangle$, com $\alpha$ totalmente positivo, são algébricos. Para tanto necessitamos de resultados auxiliares.

Lema 5.4. Sejam $K$ um corpo e $L$ uma extensão separável de $K$ de grau ímpar $m$. Para cada $\delta \in \dot{K}$, existe $\lambda \in L$ tal que $\operatorname{Tr}_{L / K}\langle\lambda\rangle=\langle\delta\rangle$ em $W(K)$.

Dem.: Sejam $x$ um elemento primitivo da extensão $L / K$ e $\left\{1, x, \ldots, x^{m-1}\right\}$ uma base de $L$ sobre $K$. Definamos a aplicação $s: L \rightarrow K$, por $s(1)=s(x)=\cdots=s\left(x^{m-\dot{2}}\right)=$ 0 e $s\left(x^{m-1}\right)=\delta$. Seja $s\langle 1\rangle: L \times L \rightarrow K$ a forma bilinear associada a $s$ dada por $s\langle 1\rangle(x, y)=s(x y)$, para todos $x, y \in L$. Assim, a matriz associada a $s\langle 1\rangle$, com relação à base $\left\{1, x, \ldots, x^{m-1}\right\}$ é:

$$
\left(\begin{array}{ccc}
0 & \cdots & \delta \\
\vdots & \delta & \\
\delta & & *
\end{array}\right)
$$

que tem determinante $\delta$ módulo $\dot{K}^{2}$, pois $m$ é ímpar. Note que a base $\left\{1, x, \ldots, x^{m-1}\right\}$ contém os vetores $\left\{1, x, \ldots, x^{\frac{m-3}{2}}\right\}$ que são isotrópicos e dois a dois ortogonais em relação 
à forma bilinear $s\langle 1\rangle$. Como um plano hiperbólico não pode conter dois vetores isotrópicos ortogonais, temos que $s\langle 1\rangle$ contém uma soma ortogonal de $\frac{m-1}{2}$ planos hiperbólicos. $\mathrm{Da}$ Observação 1.11 temos que $\langle 1,-1\rangle=0 \in(K)$, e assim $s\langle 1\rangle=\langle\delta\rangle$. Como $L / K$ é separável, de Lema 1.15, temos que $L \cong L^{*}$. Logo existe $\lambda \in L$ tal que $s=t r_{\lambda}$, ou seja, $s\langle 1\rangle=\operatorname{Tr}_{L / K}\langle\lambda\rangle$ em $W(K)$. Conseqüentemente $\operatorname{Tr}_{L / K}\langle\lambda\rangle=\langle\delta\rangle$ em $W(K)$, como queríamos.

Lema 5.5. Sejam $K$ um corpo Hilbertiano ordenado e $\delta \in \dot{K}$ totalmente positivo. Então para todo inteiro positivo $n$, existe uma extensão $L_{n} / K$ tal que

$$
\operatorname{Tr}_{L_{n} / K}\langle 1\rangle=2^{n}\langle 1\rangle \perp\left(2^{n}-1\right)\langle-\delta\rangle
$$

em $W(K)$, ou seja, $2^{n}\langle 1\rangle \perp\left(2^{n}-1\right)\langle-\delta\rangle$ é algébrico.

Dem.: Como $K$ é ordenado, temos que $\operatorname{car}(K)=0$. Provaremos o lema por indução sobre $n$. Do Corolário 5.3, temos que existe uma extensão separável $L / K$ tal que $\operatorname{Tr}_{L / K}\langle 1\rangle=\langle 1,1,-\delta\rangle$ em $W(K)$, que é exatamente o caso $n=1$. Suponhamos que $n>1$ e que o resultado seja verdadeiro para $n-1$, ou seja, existe uma extensão $L_{n-1} / K$ tal que $\operatorname{Tr}_{L_{n-1} / K}\langle 1\rangle=2^{n-1}\langle 1\rangle \perp\left(2^{n-1}-1\right)\langle-\delta\rangle \operatorname{em} W(K)$. Como $\left[L_{n-1}: K\right]=\operatorname{dim}\left(\operatorname{Tr}_{L_{n-1} / K}\langle 1\rangle\right)$ que tem a mesma paridade que $\operatorname{dim}\left(2^{n-1}\langle 1\rangle \perp\left(2^{n-1}-1\right)\langle-\delta\rangle\right)$, temos que $L_{n-1} / K$ é uma extensão de grau ímpar. Usando o Lema 5.4 para $-\delta \in \dot{K}$, temos que existe $\lambda \in L_{n-1}$ tal que $\operatorname{Tr}_{L_{n-1} / K}\langle\lambda\rangle=\langle-\delta\rangle$ em $W(K)$. Do Corolário 5.3, existe uma extensão $L_{n} / L_{n-1}$ tal que $\operatorname{Tr}_{L_{n} / L_{n-1}}\langle 1\rangle=\langle 1,1, \lambda\rangle=2\langle 1\rangle \perp\langle\lambda\rangle$ em $W(K)$. Aplicando $\operatorname{tr}_{L_{n-1} / K}$ em ambos os lados e usando o Lema 1.14 obtemos as seguintes igualdades em $W(K)$

$$
\begin{aligned}
\operatorname{Tr}_{L_{n} / K}\langle 1\rangle & =2\left(\operatorname{Tr}_{L_{n-1} / K}\langle 1\rangle\right) \perp \operatorname{Tr}_{L_{n-1} / K}\langle\lambda\rangle \\
& =2\left(2^{n-1}\langle 1\rangle \perp\left(2^{n-1}-1\right)\langle-\delta\rangle\right) \perp\langle-\delta\rangle \\
& =2^{n}\langle 1\rangle \perp\left(2^{n}-1\right)\langle-\delta\rangle,
\end{aligned}
$$

como queríamos.

Teorema 5.6. Seja $K$ um corpo Hilbertiano ordenado. Todo elemento em $W(K)$ representado por um elemento da forma $\langle\delta\rangle$, com $\delta \in \dot{K}$ totalmente positivo, é algébrico.

Dem.: Seja $\delta \in K$. totalmente positivo. De [S1] pag. 108, temos que $\delta$ pode ser escrito como uma soma de quadrados, ou seja, existe um inteiro positivo $n$ tal que a forma bilinear $2^{n}\langle 1\rangle$ representa $\delta$. Mas a forma $2^{n}\langle 1\rangle$ é uma forma de Pfister e como representa $\delta$, de [S1] pag. 143 , temos que $2^{n}\langle 1\rangle \simeq 2^{n}\langle\delta\rangle$. Usando o Lema 5.5 temos que existe uma extensão separável $L_{n} / K$ tal que $\operatorname{Tr}_{L_{n} / K}\langle 1\rangle=2^{n}\langle 1\rangle \perp\left(2^{n}-1\right)\langle-\delta\rangle$ em $W(K)$. Portanto, em $W(K)$ temos

$$
\operatorname{Tr}_{L_{n} / K}\langle 1\rangle=2^{n}\langle 1\rangle \perp\left(2^{n}-1\right)\langle-\delta\rangle=2^{n}\langle\delta\rangle \perp 2^{n}\langle-\delta\rangle \perp\langle-\delta\rangle=\langle\delta\rangle
$$

como queríamos. 
Sejam $K$ um corpo de números algébricos e $L$ uma extensão finita e separável de $K$. Se $P$ é uma ordem de $K$, de Lema 2.5 temos que $\operatorname{ass}_{P}\left(\operatorname{Tr}_{L / K}\langle 1\rangle\right) \geq 0$. Mais precisamente, essa assinatura é o número de extensões da ordem $P$ para uma ordem $P^{\prime}$ de $L$. Além disso, do Teorema 3.12 e do Corolário 3.10 seque que os corpos de números algébricos são corpos Hilbertianos ordenados de característica zero. No próximo resultado mostraremos que sobre um corpo de números algébricos, sob algumas restrições, se um elemento do anel $W(K)$ pode ser representado por uma forma traço escalar, então ele é algébrico.

Teorema 5.7. Sejam $K$ un corpo de números algébricos e $b \in W(K)$. Sejam $L / K$ uma extensão finita e separável e $\lambda \in L$ tais que $b=\operatorname{Tr}_{L / K}\langle\lambda\rangle$ em $W(K)$. Se cada ordem $P$ de $K$ se extende a exatamente $s_{P}=a s s_{P}(b)$ ordens de $L$, então $b$ é algébrico.

Dem.: Do Lema 2.8 temos que $\operatorname{ass}_{P}(b)=\operatorname{ass}_{P}\left(\operatorname{Tr}_{L / K}\langle\lambda\rangle\right)=\sum_{P^{\prime} \supseteq P} \operatorname{ass}_{P^{\prime}}(\langle\lambda\rangle)$, onde a soma é tomada sobre todas as extensões $P^{\prime}$ de $P$ para $L$. Por outro lado, $a s s_{P}(b)=s_{p}$, o que implica que ass $s_{P^{\prime}}(\langle\lambda\rangle)=1$ para toda ordem $P^{\prime}$ de $L$ que seja extensão de alguma ordem $P$ de $K$. Mas toda ordem $P^{\prime}$ de $L$ é extensão de alguma ordem $P$ de $K$. Logo $\lambda$ é também totalmente positivo em $L$. Agora, do Teorema 5.6, existe uma extensão $F / K$ tal que $\langle\lambda\rangle=T r_{F / L}\langle 1\rangle$ em $W(L)$. Aplicando $t r_{L / K}$ em ambos os lados e usando o Lema 1.14 obtemos $\operatorname{Tr}_{L / K}\langle\lambda\rangle=\operatorname{Tr}_{F / K}\langle 1\rangle$ em $W(K)$ e assim, $b=\operatorname{Tr}_{F / K}\langle 1\rangle$ em $W(K)$, ou seja, $b$ é algébrico.

Agora, estamos interessados em mostrar que sobre um corpo de números algébricos $K$, todo elemento de $W(K)$ que é representado por um espaço bilinear que tem assinatura positiva, para toda ordem de $K$, é algébrico. Para tanto, introduziremos alguns conceitos e resultados.

Seja $K$ um corpo. Um operador hermitiano sobre $K$ é uma tripla $(V, b, \sigma)$, onde $(V, b)$ é um espaço bilinear simétrico e $\sigma: V \rightarrow V$ é um operador auto-adjunto com respeito à forma bilinear $b$, isto é, $b(\sigma(x), y)=b(x, \sigma(y))$, para todo $x, y \in V$.

Lema 5.8. Sejam $(V, b) \in \mathfrak{B i l}(K), \sigma: V \rightarrow V$ um operador linear e $\mathfrak{C}$ uma base de $V$. Se $A$ a matriz de $\sigma$ na base $\mathfrak{C}$ e $B$ a matriz de $b$ em relação à mesma base $\mathfrak{C}$ de $V$ e $A^{t} B=B A$, então $\sigma$ é auto-adjunto, ou seja, $(V, b, \sigma)$ é um operador hermitiano sobre $K$.

Dem.: Sejam $x: y \in V$. Se $X$ e $Y$ são os vetores coluna das coordenadas de $x, y$ em relação à base $\mathcal{C}$. Então, na forma matricial, temos que

$$
b(\sigma(x), y)=(A X)^{t} B Y=X^{t} A^{t} B Y=X^{t} B A Y=b(x, \sigma(y)) .
$$

Logo $\sigma$ é auto-adjunto, como queríamos.

Sejam $V$ um espaço vetorial sobre um corpo $K$ de dimensão $n$, e $\sigma: V \rightarrow V$, um operador linear com polinômio característico $p(X)$ irredutível sobre $K$. Seja $L=\frac{K[X]}{(p(X))} \cong$ $K(x)$, com base $\left\{1, x, \ldots, x^{n-1}\right\}$, onde $x=X+(p(X))$. Observe que $\operatorname{dim}_{K} L=n=$ 
$\operatorname{dim}_{K} V$. Note ainda que os elementos de $L$ são da forma $f(x)=a_{0}+a_{1} x+\cdots a_{n-1} x^{n-1}$ com $a_{i} \in K$, para $0 \leq i \leq n-1$. Logo $f(\sigma)=a_{0} i d_{V}+a_{1} \sigma+\cdots a_{n-1} \sigma^{n-1}$ é um operador linear de $V$.

Com produto escalar $\bullet$ sobre $V$, definido por

$$
\begin{aligned}
\bullet: \quad & L \times V \rightarrow V \\
& (f(x), v) \mapsto f(x) \bullet v=f(\sigma)(v),
\end{aligned}
$$

para todo $v \in V$, segue facilmente que $V$ é um $L$-espaço vetorial. Além disso, como $\operatorname{dim}_{K} V=\operatorname{dim}_{L} V \operatorname{dim}_{K} L$, temos que $\operatorname{dim}_{L} V=1$. Resumimos este parágrafo em:

Lema 5.9. Sejam $V$ um espaço vetorial sobre um corpo $K, \sigma: V \rightarrow V$ um operador linear com polinômio característico irredutível $p(X) \in K[X]$ e $L=\frac{K[X]}{(p(X))}$. Então $V$ é um $L$-espaço vetorial de dimensão 1.

O próximo resultado nos diz que todo operador hermitiano cujo operador auto-adjunto tem polinômio característico irredutível e separável pode ser representado no anel de Witt por uma forma traço escalar.

Proposição 5.10. Seja $(V, b, \sigma)$ um operador hermitiano sobre um corpo $K$. Se o polinômio característico $p(X)$ de $\sigma$ sobre $K$ é irredutível e separável, então existe uma extensão $L / K$ e $\lambda \in L$ tal que $(V, b)=\operatorname{Tr}_{L / K}(\langle\lambda\rangle)$ em $W(K)$.

Dem.: Desde que $p(X)$ é irredutível, do Lema 5.9, temos que $V$ é um $L$-espaço vetorial de dimensão 1 , onde $L=\frac{K[X]}{(p(X))}$.

Começaremos mostrando que podemos definir uma forma $L$-bilinear e simétrica

$$
B: V \times V \rightarrow L
$$

satisfazendo $\operatorname{tr}_{L / K}(B(v, w))=b(v, w)$, para todos $v, w \in V$. Desde que $L / K$ é separável, temos do Lema 1.15, que a adjunta de $\operatorname{Tr}_{L / K}\langle 1\rangle$ é um isomorfismo, ou seja, $L \cong L^{*}$. Dado $(v, w) \in V \times V$ a aplicação $f_{(v, w)}: L \rightarrow K$, definida por $f_{(v, w)}(a)=b(a x, y)$ é $K$-linear, ou seja, $f_{(v, w)} \in L^{*}$. Logo, existe $\lambda_{(v, w)} \in L$ tal que $f_{(v, w)}$ é a imagem de $\lambda_{(v, w)}$ pela adjunta de $\operatorname{Tr}_{L / K}\langle 1\rangle$ e, conseqüentemente, $\operatorname{tr}_{L / K}\left(\lambda_{(v, w)} a\right)=f_{(v, w)}(a)=b(a x, y)$, para todo $a \in L$.

Definimos, então $B: V \times V \rightarrow L$, por $B(v, w)=\lambda_{(v, w)}$, para cada $(v, w) \in V \times V$.

Agora, mostraremos que $B$ é simétrica. Para tanto, sejam $v, w \in V, B(v, w)=z \mathrm{e}$ $B(w, v)=t$. Então $\operatorname{tr}_{L / K}(a z)=\operatorname{tr}_{L / K}(a B(v, w))=b(a v, w)$ e $\operatorname{tr}_{L / K}(a t)=$ $\operatorname{tr}_{L / K}(a B(w, v))=b(a w, v)$, para todo $a \in L$.

Como $a \in L=\frac{K[X]}{(p(X))}$, temos que $a=a_{0}+a_{1} x+\cdots a_{n-1} x^{n-1}$, onde $x=X+(p(X))$ e $n=g r(p(X))$. Da estrutura de $L$-espaço vetorial de $V$ temos que $a v=a \cdot v=$ $a_{0} v+\cdots+a_{n-1} \sigma^{n-1}(v)$, para todo $v \in V$ e $a \in L$. Logo, usando que $\sigma$ é auto-adjunto e que $b$ é $K$-bilinear, obtemos 


$$
\begin{aligned}
b(a v, w) & =b\left(a_{0} v+\cdots+a_{n-1} \sigma^{n-1}(v), w\right)=a_{0} b(v, w)+\cdots+a_{n-1} b\left(v, \sigma^{n-1}(w)\right) \\
& =b\left(v, a_{0} w\right)+\cdots+b\left(v, a_{n-1} \sigma^{n-1}(w)\right)=b(v, a w)=b(a w, v) .
\end{aligned}
$$

Assim, $\operatorname{tr}_{L / K}(a z)=b(a v, w)=b(a w, v)=\operatorname{tr}_{L / K}(a t)$, ou seja, $0=\operatorname{tr}_{L / K}(a(z-t))=$ $\operatorname{Tr}_{L / K}(a, z-t)$, para todo $a \in L$. Do Lema 1.15 temos que $z=t$, e $B$ é simétrica.

Agora, desde que $B$ é simétrica, para mostrarmos que $B$ é $L$-bilinear, é suficiente mostrar que $B$ é $L$-linear em uma das variáveis. Para tanto, considere $v, w \in V$ e $a \in L$. Para todo $c \in L$, temos $\operatorname{tr}_{L / K}(c a B(v, w))=b((c a) v, w)=b(c(a v), w)=$ $\operatorname{tr}_{L / K}(c B(a v, w))$, ou seja, $\operatorname{tr}_{L / K}(c(a B(v, w)-B(a v, w))=0$ e novamente do Lema 1.15 temos que $a B(v, w)=B(a v, w)$, para todo $a \in L$ e $v, w \in V$.

Para todo $v, w, u \in V$, sejam $B(v+u, w)=z, B(v, w)=t$ e $B(u, w)=s$. Então $\operatorname{tr}_{L / K}(a z)=b(a(v+u), w)=b(a v+a u, w)=b(a v, w)+b(a u, w)=\operatorname{tr}_{L / K}(a t)+\operatorname{tr}_{L / K}(a s)=$ $\operatorname{tr}_{L / K}(a(t+s))$, ou seja, $\operatorname{tr}_{L / K}(a(z-(t+s)))=0$, para todo $a \in L$. Usando novamente o Lema 1.15 , obtemos que $B$ é $L$-linear na primeira variável.

Assim, $\operatorname{tr}_{L / K}(B(v, w))=b(v, w)$, para todo $v, w \in V$. Como $V$ é um $L$-espaço vetorial de dimensão 1 , existe $v_{0} \in L$ tal que $\theta: L \rightarrow V$, definida por $\theta(s)=s v_{0}$ é um $L$-isomorfismo. Tomando $\lambda=B\left(v_{0}, v_{0}\right)$, temos que $\lambda \neq 0$, pois caso contrário, para todo $v, w \in V$, existem $s, s^{\prime}$ tais que $v=s v_{0}, w=s^{\prime} v_{0}$. Logo $b(v, w)=\operatorname{tr}_{L / K}(B(v, w))=$ $\operatorname{tr}_{L / K}\left(s s^{\prime} 0\right)=0$, o que contradiz o fato que $(V, b)$ é não degenerado. Portanto, para $v, w \in V$, com $v=s v_{0}$ e $w=s^{\prime} v_{0}$, temos $b(v, w)=\operatorname{tr}_{L / K}(B(v, w))=\operatorname{tr}_{L / K}\left(\lambda s s^{\prime}\right)=$ $\operatorname{Tr}_{L / K}(\langle\lambda\rangle)\left(s, s^{\prime}\right)$, ou seja, $\theta: L \rightarrow V$ é uma isometria de $L$-espaços bilineares e, como queríamos, $(V, b) \simeq \operatorname{Tr}_{L / K}(\langle\lambda\rangle)$.

Lema 5.11. Sejam $S$ uma matriz diagonal de ordem $n$ sobre um corpo de números algébricos $K$ e $P$ uma ordem de $K$. Seja $m$ o número de elementos negativos na diagonal de $S$. Então existe uma matriz simétrica $C$ de ordem $n$ sobre $K$ tal que $S C$ tem polinômio característico $p(X)$ irredutível sobre $K$. Além disso, $p(X)$ tem exatamente $s_{p}=n-2 m$ raízes em $K$.

Dem.: Consideremos inicialmente a matriz simétrica $n \times n$ de indeterminadas $C=\left(X_{i j}\right)$. Então do Lema 4.5 temos que $p\left(\left\{X_{i j}\right\}, X\right)=\operatorname{det}\left(C S-X I_{n}\right)$ é irredutível em $K\left[\left\{X_{i j}\right\}, X\right]$. Denote os elementos da diagonal de $S$ por $s_{1}, \ldots, s_{n}$. Por hipótese, $m$ desses elementos são negativos, digamos $s_{2}, s_{4}, \ldots, s_{2 m}$. Tome

$$
\left(\lambda_{i j}\right)=\left(\begin{array}{cccccc}
B_{1} & 0_{2} & 0_{2} & \cdots & \cdots & 0_{2} \\
0_{2} & \ddots & 0_{2} & \cdots & \cdots & 0_{2} \\
\vdots & \cdots & B_{m} & 0_{2} & \cdots & 0_{2} \\
0 & \cdots & 0 & c_{2 m+1} & 0 & 0 \\
\vdots & \cdots & \cdots & 0 & \ddots & 0 \\
0 & \cdots & \cdots & \cdots & 0 & c_{n}
\end{array}\right) \in M_{n}(K)
$$


onde $B_{i}=\left(\begin{array}{ll}0 & 1 \\ 1 & 0\end{array}\right)$ para todo $i=1, \ldots, m$ e $0_{2}=\left(\begin{array}{ll}0 & 0 \\ 0 & 0\end{array}\right)$, tal que os elementos $c_{i} s_{i}$ sejam distintos, que existe porque $K$ é um corpo infinto, temos $p\left(\left\{\lambda_{i j}, X\right\}\right)=$ $(-1)^{2 m}\left(c_{2 m+1} d_{2 m+1}-X\right) \cdots\left(c_{n} d_{n}-X\right)$, e é um polinômio separável de $K[X]$, com $s_{P}=$ $n-2 m$ raízes em $K$.

Uma ordem $P$ de $K$ induz uma topologia em $K$ e, conseqüentemente em $K^{n^{2}}$. Como $p\left(\left\{\lambda_{i j}, X\right\}\right)$ tem $s_{P}$ raízes distintas em $K$, temos que existem vizinhanças dessas raízes que não contém outras raízes de $p\left(\left\{\lambda_{i j}, X\right\}\right)$. Logo existem matrizes $\left(\lambda_{i j}^{\prime}\right)$ sufientemente próximas de $\left(\lambda_{i j}\right)$ tais que $p\left(\left\{\lambda_{i j}, X\right\}\right)$ também tem $s_{p}$ raízes distintas em $K$. O conjunto de tais matrizes formam um aberto em $U_{P}$ na topologia de $K^{n^{2}}$ por $P$. Desde que $K$ é Hilbertiano, por um resultado de Dries contido em [D], temos que $U \cap H_{K}\left(p\left(\left\{X_{i j}\right\}, X\right)\right) \neq$ $\emptyset$. Logo existe $\left(\alpha_{i j}\right) \in M_{n}(K)$ tal que $\left.p\left(\left\{\alpha_{i j}\right\}, X\right)\right)$ é irredutível e separável em $K[X]$ com exatamente $s_{p}$ raízes.

Teorema 5.12. Seja $K$ um corpo de números algébricos. Todo elemento $(V, b) \in W(K)$ com

$$
s_{P}=a s s_{P}(b) \geq 0,
$$

para todas as ordens $P$ de $K$, é algébrico.

Dem.: Seja $(V, b) \in W(K)$. Como $\operatorname{car}(K)=0$, do Terorema 1.5 podemos supor que $b$ é diagonalizável, ou seja, $b=\left\langle s_{1}, \ldots, s_{n}\right\rangle$. Seja $S=\operatorname{diag}\left(s_{1}, \ldots, s_{n}\right)$ a matriz diagonal correspondente. Do Lema 5.11 temos que existe uma matriz simétrica $C$ tal que $A=C S$ tem polinômio característico $p$ irredutível com exatamente $s_{P}=a s s_{P}(b)$ raízes reais. Além disso, temos que $A^{t} S=S^{t} C^{t} S=S C S=S A$. Do Lema 5.8, temos que $A$ representa um operador auto-adjunto. Logo, do Lema 4.3 e da Proposição 4.4 temos que existe $\lambda \in L=\frac{K[X]}{(p(X))}$, tal que $b=\operatorname{Tr}_{L / K}(\langle\lambda\rangle)$ em $W(K)$. Como $p$ tem exatamente $s_{p}$ raízes reais, temos do Teorema 2.2 que qualquer ordem $P$ de $K$ se estende a exatamente $s_{p}$ ordens de $L$. Agora, o resultado segue do Lema 5.7.

Encerraremos este trabalho mostrando como obter o Teorema 4.6, usando operadores hermitianos sobre $K$. Para tanto, precisamos do seguinte resultado preliminar.

Lema 5.13. Seja $K$ um corpo hilbertiano. Sejam $(V, b) \in \mathfrak{B i l}(K), \mathcal{B}=\left\{v_{1}, \ldots, v_{n}\right\}$ uma base de $V$ sobre $K$ e $S$ a matriz de $b$ em relação à base $\mathcal{B}$. Então existe uma matriz simétrica $C$, tal que $A=C S$ tem polinômio característico irredutível e separável sobre $K$. Além disso, $A$ representa um operador auto-adjunto de $V$, com respeito a forma bilinear b.

Dem.: Como característica de $K$ é distinta de 2, podemos supor que $S$ é diagonal, ou seja, $S=\operatorname{diag}\left(s_{1}, \ldots, s_{n}\right)$. Seja $A^{\prime}=\left(X_{i j}\right)$ uma matriz simétrica $n \times n$ de indeterminadas. 
Do Lema 4.5 temos que $p\left(\left\{X_{i j}\right\}, X\right)=\operatorname{det}\left(A-S^{-1}\left(X I_{n}\right)\right)$ é um polinômio irredutível en $K\left[\left\{X_{i j}\right\}, X\right]$.

Além disso, escrevendo $X_{i j}=\delta_{i j} Y_{i}$, onde $\delta_{i j}$ é o delta de Kronecker, temos que $h\left(Y_{1}, \ldots, Y_{n}, X\right)=\prod_{i=1}^{n}\left(Y_{i}-s_{i}^{-1} X\right)$ é o produto da diagonal principal de $\left(A-S^{-1}\left(X I_{n}\right)\right)$. Mais ainda, $h$ é um polinômio separável na variável $X$, pois as raízes de $h$ são $Y_{i} s_{i}$, para $i=1, \ldots, n$, que são todas distintas. Logo $\frac{\partial h}{\partial X}$ é não nula e tem grau $n-1$. Escrevendo $p\left(\left\{X_{i j}\right\}, X\right)=h\left(\left\{X_{i j}\right\}, X\right)+\sum_{l=0}^{n-1} f_{l}\left(\left\{X_{i j}\right\}\right) X^{l} \in K\left[\left\{X_{i j}\right\}, X\right]$, temos que

$$
\frac{\partial p}{\partial X}\left(\left\{X_{i j}\right\}, X\right)=\frac{\partial h}{\partial X}\left(\left\{X_{i j}\right\}, X\right)+\sum_{l=1}^{n-1} l f_{l}\left(\left\{X_{i j}\right\}\right) X^{l-1} .
$$

Desde que $g r\left(\frac{\partial X^{l} f_{l}}{\partial X}\right) \leq n-2$ para $l=0, \ldots, n-1$ e $\operatorname{gr}\left(\frac{\partial f}{\partial X}\right)=n-1$, podemos concluir que $\frac{\partial p}{\partial X}$ é não nula.

Considere o seguinte subconjunto de Hilbert de $K$, $H_{K}\left(p ; \frac{\partial p}{\partial X}\right)=\left\{\left(\lambda_{i j}\right) \in M_{n}(K) ; p\left(\left\{\lambda_{i j}\right\}, X\right)\right.$ é irredutivel em $K[X]$ e $\left.\frac{\partial p}{\partial X}\left(\left\{\lambda_{i j}\right\}, \alpha\right) \neq 0\right\}$. Como $K$ é Hilbertiano, temos que $H_{K}\left(p ; \frac{\partial p}{\partial X}\right) \neq \emptyset$, ou seja, existe uma matriz simétrica $C=\left(\lambda_{i j}\right) \in M_{n}(K)$ tal que $p\left(\left\{\lambda_{i j}\right\}, X\right)$ é irredutível e $\frac{\partial p}{\partial X}\left(\left\{\lambda_{i j}\right\}, \alpha\right) \neq 0$, ou seja, $\frac{\partial p}{\partial X}\left(\left\{\lambda_{i j}\right\}, X\right)$ e um polinômio não nulo em $K[X]$.

Note que para $A=C S$ temos que $A^{t} S=(C S)^{t} S=S^{t}(C)^{t} S=S(C) S=S A$. Do Lema 5.8, temos que $A$ representa um operador auto-adjunto de $V$ com respeito à forma bilinear $b$.

Finalmente, o Teorema 4.6 obtido usando operadores hermitianos.

Teorema 5.14. Seja $K$ um corpo hilbertiano. Então qualquer forma bilinear não degenerada sobre $K$ é isométrica a uma forma traço escalar.

Dem.: Seja $(V, b) \in \mathfrak{B i l}(K)$, com $\operatorname{dim} V=n$. Do lema anterior, existe uma matriz simétrica $C \in M_{n}(K)$ tal que $A=C S$ tem polinômio característico irredutível e separável sobre $K$ e $A$ representa um operador auto-adjunto de $V$ com relação à $b$, onde $S$ é a matriz de $b$ em relação a alguma base ortogonal de $V$. Assim, $\left(V, b, \sigma_{A}\right)$ é um operador hermitiano sobre $K$, onde $\sigma_{A}$ é o operador auto-adjunto representado por $A$. Agora, da Proposição 5.10 , temos que $b=\operatorname{Tr}_{L / K}(\langle\lambda\rangle)$, onde $L=\frac{K[X]}{(p(X))}, \operatorname{com} p(X)$ é o polinômio característico de $A$ e $\lambda \in L$. Como $\operatorname{dim} b=n$ e $\operatorname{dim} \operatorname{Tr}_{L / K}(\langle\lambda\rangle)=\operatorname{dim}_{K} L=n$, do Teorema 1.12, temos que $b \simeq \operatorname{Tr}_{L / K}(\langle\lambda\rangle)$, como queríamos. 


\section{Referências Bibliográficas}

[C-P] Conner, P. E. and Perlis, R; A survey of trace forms of algebraic number fields; Series in Pure Math, V.2, World Scientific, 1984.

[D] Dries. L.v.d.; Model theory of fields; Thesis, Utrecht, 1978.

[F] Fried, M. D. and Jarden, M; Field Arithmetic; Springer-Verlag, 1986.

[H] Hefez, A; Singularidas de Curvas Irredutiveis Planas; The Sixth Workshop on Real and Complex Singularities, USP-São Carlos, 2000.

[K] Krüskemper, M. and Scharlau, W.; On trace forms of hilbertian fields; Comment. Math. Helvetici, 63, 296-304, 1988.

[L] Lang, S.; Algebra; Addison-Wesley Publishing Company, Inc., 3rd ed., 1993.

[Lam] Lam, T. Y.; The algebraic theory of quadratic forms; University of California, 1973.

[S1] Scharlau, W.; Quadratic and Hermitian Forms; Springer-Verlag, 1985.

[S2] Scharlau, W; On trace forms of algebraic number fields ; Math. Z. 196, 125-127, 1987.

[Se] Serre, J.-P.; The Witt invariant of the form $\operatorname{Tr}\left(x^{2}\right)$; Comment. Math . Helv. 59, 651-676, 1984.

[St] Stewart, I.; Galois Theory; Chapman and Hall, London, 1973.

[T] Taussky-Todd, O.; The discriminat matrices of an algebraic number field; J. London Math. Soc. 43, 1968.

[V] Vila, N.; Sobre el Teorema D'irreductibilitat de Hilbert; Pub. Mat. UAB. Vol $30 n^{\circ}$ 2-3, 83-87, 1986. 2.

[W] Waterhouse, W. C.; Scaled trace forms over number fields; Arch. Math. 47, 229-231, 1986. 
